\title{
Heavy Metal Adsorption Using Magnetic Nanoparticles for Water Purification: A Critical Review
}

\author{
Christos Liosis ${ }^{1}\left(\mathbb{D}\right.$, Athina Papadopoulou ${ }^{2}$, Evangelos Karvelas ${ }^{3,4}\left(\mathbb{D}\right.$, Theodoros E. Karakasidis ${ }^{4, *(D)}$ \\ and Ioannis E. Sarris ${ }^{3}$ (D) \\ 1 Department of Civil Engineering, University of Thessaly, 38334 Volos, Greece; cliosis@uth.gr \\ 2 Inorganic Chemistry Laboratory, Department of Chemistry, National and Kapodistrian University of Athens, \\ 15771 Athens, Greece; athinapapad@chem.uoa.gr \\ 3 Department of Mechanical Engineering, University of West Attica, 12243 Athens, Greece; \\ karvelas@uth.gr (E.K.); sarris@uniwa.gr (I.E.S.) \\ 4 Condensed Matter Physics Lab, Department of Physics, University of Thessaly, 35100 Lamia, Greece \\ * Correspondence: thkarak@uth.gr; Tel.: +30-22310-60280
}

check for updates

Citation: Liosis, C.; Papadopoulou, A.; Karvelas, E.; Karakasidis, T.E.; Sarris, I.E. Heavy Metal Adsorption Using Magnetic Nanoparticles for Water Purification: A Critical Review. Materials 2021, 14, 7500. https:// doi.org/10.3390/ma14247500

Academic Editors: Fernando Gomes de Souza Junior and Francisco Javier Guzmán Bernardo

Received: 17 September 2021

Accepted: 1 December 2021

Published: 7 December 2021

Publisher's Note: MDPI stays neutral with regard to jurisdictional claims in published maps and institutional affiliations.

Copyright: (c) 2021 by the authors. Licensee MDPI, Basel, Switzerland. This article is an open access article distributed under the terms and conditions of the Creative Commons Attribution (CC BY) license (https:/ / creativecommons.org/licenses/by/ $4.0 /)$.

\begin{abstract}
Research on contamination of groundwater and drinking water is of major importance. Due to the rapid and significant progress in the last decade in nanotechnology and its potential applications to water purification, such as adsorption of heavy metal ion from contaminated water, a wide number of articles have been published. An evaluating frame of the main findings of recent research on heavy metal removal using magnetic nanoparticles, with emphasis on water quality and method applicability, is presented. A large number of articles have been studied with a focus on the synthesis and characterization procedures for bare and modified magnetic nanoparticles as well as on their adsorption capacity and the corresponding desorption process of the methods are presented. The present review analysis shows that the experimental procedures demonstrate high adsorption capacity for pollutants from aquatic solutions. Moreover, reuse of the employed nanoparticles up to five times leads to an efficiency up to $90 \%$. We must mention also that in some rare occasions, nanoparticles have been reused up to 22 times.
\end{abstract}

Keywords: adsorption; contamination; magnetic nanoparticles; heavy metals

\section{Introduction}

Nowadays, water issues, such as exhaustion of resources and quality of drinking water, have attracted the interest not only of researchers but also of national and international organizations and governments [1]. Terms such as water stress and water scarcity are now subjects of research on a daily basis. The first term refers to situations where the quantity of available water is not sufficient for agricultural, industrial, or domestic uses. It takes into account several physical aspects related to water resources, such as water quality, environmental flows, and water accessibility [2-4]. On the other hand, the second term refers to the volumetric abundance of water supply [5-7], which is the ratio of human water consumption to available water supply for a specific area [8,9]. The main factors that cause water crisis in the long run are population growth, expansion of industrial activities, urbanization, climate change, depletion of aquifers, and water contamination [10-12]. It is obvious that the coverage of global demands for safe drinking water in the near future is utopian, if we consider that water quantity is nearly constant due to the hydrologic cycle in various forms, such as seawater, groundwater, surface water, and rainwater $[13,14]$. However, climate change will affect the quality and quantity of potentially available drinking water due to increased flooding, more severe droughts, and enhanced toxicity of chemical contaminants in the environment $[15,16]$. Of more immediate concern is the efficiency of existing water treatment methods due to increasing pollution resulting from the anthropogenic activities [17]. Thus, purification of water from polluted sources is essential to enable the utilization of sustainable global water $[18,19]$. 
Water pollutants are categorized into point source, where the pollution originates from a single and identifiable source, and nonpoint source, where pollutants originate from a variety of sources [20]. Based on their main physicochemical characteristics, they can also be classified into radioactive, thermal, microorganism, nutrient, suspended solid and sediment, and organic and inorganic pollutants [21]. Inorganic pollutants consist of heavy metals, fertilizers, sulphides, ammonia, oxides of nitrogen, acids, and bases [22-24]. Since water quality is improving with increasing advances in technologies [17] and water purification is highly required to prevent toxic effects and disruption of ecological balance, this particular research review focuses on water contamination by heavy metals and ways of heavy metal removal.

Pollutants can contaminate water through both natural processes and anthropogenic activities $[25,26]$. Their concentrations in water depend on the local geological, hydrogeological, and geochemical characteristics of the aquifer. A significant burden on water quality and, consequently, public health is the increasing concentrations of heavy metals due to their toxicity, persistence, and bioaccumulative nature $[25,27]$. Unlike organic contaminants, heavy metals, which are toxic and mostly carcinogenetic, are not biodegradable and tend to accumulate in organisms [28]. Hence, exposure to toxic heavy metal through drinking water has long been a critical public health concern across the world [29]. Elements whose density exceeds $5 \mathrm{~g} / \mathrm{cm}^{3}$ fall into the category of heavy metals [30] and are listed in Table 1 based on their toxicity, which is related to the maximum contaminant level (MCL) [31]. Specific differences in metal ion toxicities arise from differences in solubility, absorbability, transport, chemical reactivity, and the complexes that are formed within the body [32].

Table 1. Metal toxicity [31,33].

\begin{tabular}{cc}
\hline Heavy Metals & MCL $(\mathbf{m g} / \mathbf{L}) \times \mathbf{1 0}^{\mathbf{2}}$ \\
\hline Zinc (Zn) & 80 \\
Nickel (Ni) & 20 \\
Copper (Cu) & 25 \\
Chromium (Cr) & 5 \\
Arsenic (As) & 5 \\
Cadmium (Cd) & 1 \\
Lead (Pb) & 0.06 \\
Mercury (Hg) & 0.003 \\
\hline
\end{tabular}

Heavy metals are classified into essential $(\mathrm{Zn}, \mathrm{Cu}, \mathrm{Fe}$, and $\mathrm{Co})$ and nonessential $(\mathrm{Cd}$, $\mathrm{Hg}$, As, and $\mathrm{Cr}$ ) based on their toxicity. At low concentrations, essential heavy metals are harmless, unlike nonessential metals, which are highly toxic [34,35]. It is worth noting that for nonessential heavy metals, toxicity is very high, even at low concentrations [36,37]. Water contamination by heavy metals occurs in anthropogenic activities or in natural processes. Sources of contamination include metal corrosion, atmospheric deposition, soil erosion of metal ions, leaching of heavy metals, sediment resuspension, and metal evaporation from water resources to soil and groundwater. Phenomena such as weathering and volcanic eruptions fall into the category natural contamination [38]. In contrast, the major sources resulting from anthropogenic activities are mining wastes, landfill leaches, municipal wastewater, urban runoff, industrial wastewater, electroplating, and electronic and metal finishing industries $[39,40]$. Moreover, due to an increasing production of metals from technological activities, the problem of waste disposal has become of paramount importance; hence many aquatic environments face metal concentrations that do not meet water quality criteria designed to protect the environment, animals, and humans [41]. Metals such as $\mathrm{Cu}, \mathrm{Cd}, \mathrm{Cr}, \mathrm{Hg}$, and $\mathrm{Ni}$ have the ability to produce reactive radicals, resulting in DNA damage, lipid peroxidation, depletion of protein sulfhydryls, and other effects [42]. In this context, the availability and release of pollutants from anthropogenic sources needs to be considered [43]. The source of pollution and the effect of each heavy metal on human health are presented in Table 2. 
Table 2. Heavy metal characteristics [16,33,44-46].

\begin{tabular}{|c|c|c|}
\hline Heavy Metal & Human Health Impacts & Common Sources \\
\hline Arsenic & $\begin{array}{l}\text { Skin damage, circulatory } \\
\text { system issues, protein } \\
\text { coagulation, nerve } \\
\text { inflammation, muscle } \\
\text { weakness, carcinogenicity }\end{array}$ & $\begin{array}{c}\text { Naturally occurring, electronic } \\
\text { production, agricultural applications, } \\
\text { nonferrous smelters, metallurgy, coal-fired } \\
\text { and geothermal electrical generation, } \\
\text { tanning, pigments, antifouling paints, } \\
\text { light filters, fireworks, veterinary } \\
\text { medicine }\end{array}$ \\
\hline Cadmium & $\begin{array}{c}\text { Kidney damage, } \\
\text { carcinogenicity, DNA damage, } \\
\text { gastrointestinal irritation, } \\
\text { hyperactivity, renal failure }\end{array}$ & $\begin{array}{l}\text { Naturally occurring, various chemical } \\
\text { industries, agricultural applications } \\
\text { (phosphatic fertilizers), pigments, } \\
\text { anticorrosive metal coatings, plastic } \\
\text { stabilizers, alloys, coal combustion }\end{array}$ \\
\hline Chromium & $\begin{array}{l}\text { Allergic dermatitis, diarrhoea, } \\
\text { nausea, vomiting, headache, } \\
\text { neurotoxicity, kidney and } \\
\text { liver damage }\end{array}$ & $\begin{array}{l}\text { Naturally occurring, steel manufacturing } \\
\text { metallurgy, refractory, chemical industries, } \\
\text { plating, pigments, textile and leather } \\
\text { tanning, passivation of corrosion of } \\
\text { cooling circuits, wood treatment }\end{array}$ \\
\hline Copper & $\begin{array}{l}\text { Gastrointestinal issues, liver } \\
\text { and kidney damage, anorexia, } \\
\text { Wilson's disease }\end{array}$ & $\begin{array}{l}\text { Household plumbing systems, naturally } \\
\text { occurring, chemical and pharmaceutical } \\
\text { equipment, pigments, alloys }\end{array}$ \\
\hline Lead & $\begin{array}{l}\text { Kidney damage, reduced } \\
\text { neural } \\
\text { development, carcinogenicity, } \\
\text { high blood pressure }\end{array}$ & $\begin{array}{l}\text { Lead-based products (batteries), } \\
\text { household plumbing systems, antiknock } \\
\text { agents, pigments, glassware, ceramics, } \\
\text { plastic, alloys, sheets, cable sheathings, } \\
\text { solder }\end{array}$ \\
\hline Mercury & $\begin{array}{l}\text { Kidney damage, nervous } \\
\text { system damage, } \\
\text { carcinogenicity, gingivitis, } \\
\text { stomatitis, gastrointestinal } \\
\text { issues, abortions }\end{array}$ & $\begin{array}{l}\text { Fossil fuel combustion, electronic } \\
\text { industries, fluorescent light bulbs, } \\
\text { electrical and measuring apparatus, } \\
\text { catalysts, pharmaceuticals, dental fillings, } \\
\text { scientific instruments, rectifiers, } \\
\text { oscillators, solders }\end{array}$ \\
\hline Nickel & $\begin{array}{l}\text { Allergic dermatitis, nausea, } \\
\text { chronic asthma, coughing, } \\
\text { carcinogenicity, hair loss }\end{array}$ & $\begin{array}{l}\text { Paper products, fertilizer plating, } \\
\text { electroplating, batteries, arc welding, rods, } \\
\text { pigments for paints and ceramics, surgical } \\
\text { and dental prostheses, moulds for ceramic } \\
\text { and glass containers, computer } \\
\text { components, catalysts }\end{array}$ \\
\hline Zinc & $\begin{array}{c}\text { Depression, lethargy, } \\
\text { neurological signs, increased } \\
\text { thirst, hyperactivity, physical } \\
\text { dysfunction }\end{array}$ & $\begin{array}{l}\text { Mining, coal, waste combustion, steel } \\
\text { processing, agricultural applications } \\
\text { (phosphatic fertilizers), anticorrosion } \\
\text { coating, batteries, cans, PVC stabilizers, } \\
\text { medicines and chemicals, rubber industry, } \\
\text { paints, soldering and welding fluxes }\end{array}$ \\
\hline
\end{tabular}

However, the removal of heavy metal ions from water has been a vital and challenging issue for several decades [47,48], without reaching the heart of the problem. On the other hand, in recent years nanotechnology has been integrated with several novel techniques for the removal of heavy metals from water systems to improve removal efficiency [49-51].

Several water purification methods for heavy metals exist, such as chemical precipitation and coagulation, flocculation, electrochemical methods, photocatalytic degradation, membrane filtration, ion exchange, bioremediation, and adsorption, just to mention few $[16,52,53]$. The present work focuses on the adsorption methods developed during the last decade, where nanotechnology contributed quite a lot to great progress. In this context, adsorption capacity and reuse efficiency of magnetic nanoparticles for capturing heavy metals in water environments are investigated. In addition, the findings are analysed based on certain characteristics, such as viability method, time of purification, water quality after purification (adsorption capacity), and nanoparticle reusability. 
The main interest in the adsorption method is the interaction between nanoparticles and adsorbents, which depends on their physicochemical properties [54]. The term physicochemical covers the particle size, surface area, surface charge, agglomeration, morphology, surface coating, and so forth. Particles with sizes below $100 \mathrm{~nm}$ are defined as nanoparticles [55], and their applications vary according to their size [54,56,57]. Magnetic, mechanical, optical, and electrical properties affect the formation and aggregation of nanoparticles [44]. Aggregation of nanoparticles is affected by surface charge, particle size, and composition. Nanoparticles without magnetic properties have limited applicability in water purification due to the difficulty of separation from the aqueous solution [58]. Magnetic nanoparticles present advantages due to their large surface area, size, and shapedependent catalytic properties and can be separated from the aqueous solution with the use of a magnet field [48,59]; thus many methods have been investigated for their potential application in both environmental and biological fields [60-62].

Our focus was on studies that have been published during the last decade. The selection of relevant research articles was performed in several stages. In the first stage, the search was based on article title, abstract, and keywords. The terms for the search were (a) water purification, (b) magnetic nanoparticles, (c) heavy metals, and (d) reusability. In the second stage, we selected articles according to purification method (adsorption process). The final stage included the expansion of the bibliography using the remaining articles' reference lists.

The sections below exhaustively describe the procedure for the adsorption method, which includes synthesis and characterization of the nanoparticles, the evaluation strategies, and the main findings, such as adsorption capacity and reusability.

\section{Adsorption Method}

The adsorption process occurs between a porous solid medium and a multicomponent fluid or gas by the formation of physical or chemical bonds and offers flexibility in design and operation, which is combined with very satisfying results (adsorption efficiency) [63]. Moreover, due to the reversibility of the adsorption mechanism, adsorbents can be regenerated through suitable desorption processes [28].

The term adsorption refers to a mass transfer process where the pollutants in a solution are transferred to a solid adsorbent [64], which is frequently used for water and wastewater purification under the principles of pore filling, $\mathrm{H}$ bonding, hydrophobic interaction, and ion exchange $[65,66]$. The process under physical or chemical techniques contains many different adsorption forces that can effectively adsorb specific pollutants [67]. Physical adsorption is due to weak Van der Waals forces of attraction, and chemical adsorption is due to the strong covalent bond between the adsorbent and the adsorbate [34]. In aqueous solutions, iron oxide hydroxyl is functionalized because of the Fe atoms that coordinate with water. Iron oxide surface atoms act as Lewis acids and coordinate with molecules that donate lone-pair electrons. These hydroxyl groups are amphoteric and may react with acids or bases $[68,69]$. Based on the physicochemical properties of magnetic nanoparticles and due to chemisorption and/or physisorption, we can achieve the adsorption of heavy metals from contaminated water. Terms such as adsorption capacity and removal efficiency are employed for the quantification of the adsorption process. The adsorption capacity is enhanced due to the increased surface atoms, which appear more active and unstable and offer more unsaturated bonds [67]. Unfortunately, the efficiency of conventional adsorbents is often limited by the surface area or active sites and the adsorption kinetics [66]. A schematic representation of the water purification cycle is presented in Figure 1. 

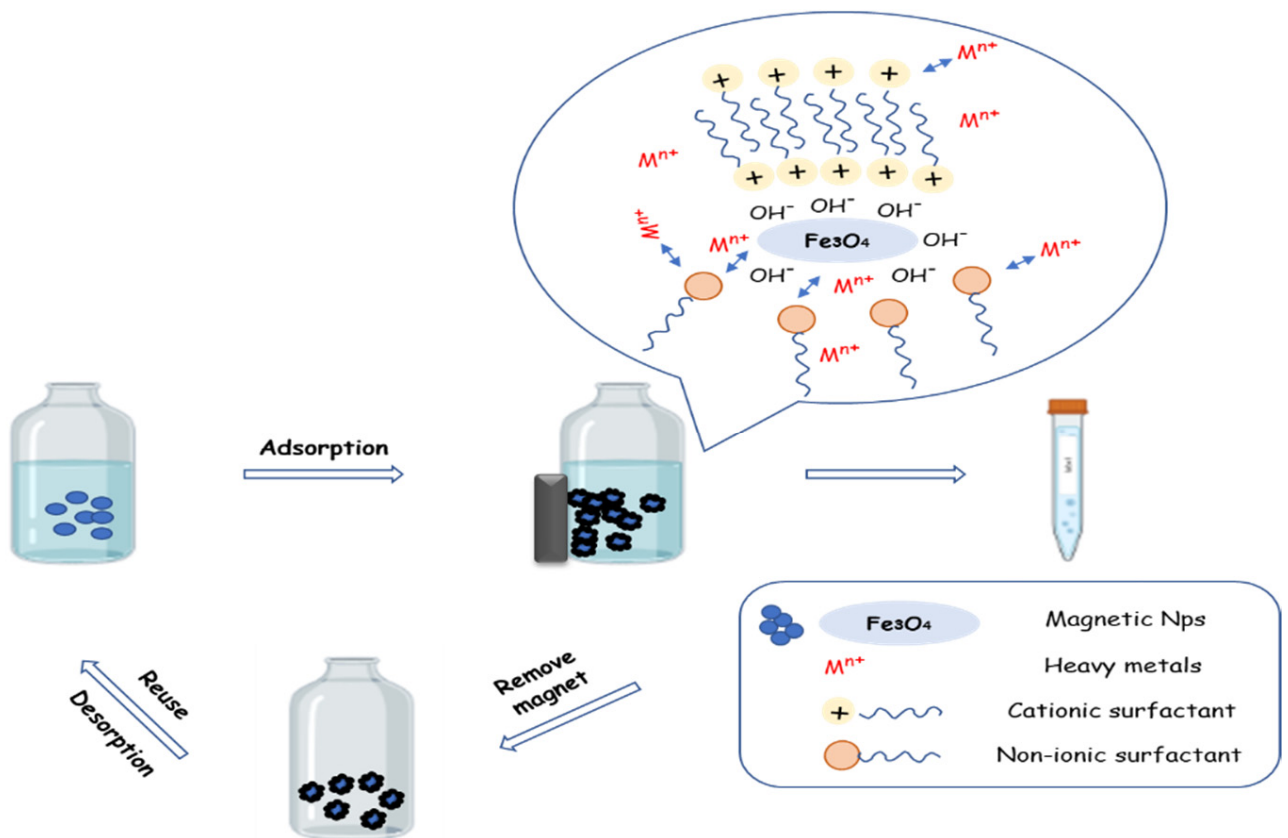

Figure 1. Schematic representation of the water purification cycle.

Based on the selected articles, Figures 2 and 3 were implemented. Despite the fact that the adsorption method of using bare or modified iron oxide nanoparticles in polluted aqua systems has already been used for several years, the scientific interest remains constant, as we can see in Figure 2. In Figure 3, we can see the research interest per year in each type of heavy metal ion. Furthermore, from Figure 3 we can see the total interest in each heavy metal during the last decade. It is evident that As (III) and Cr (III) constitute the minority of the heavy metals that have been studied by using the adsorption method. On the other hand, $\mathrm{Pb}$ (II), $\mathrm{Cu}$ (II), and $\mathrm{Cr}$ (VI) constitute the majority of the research interest, with $\mathrm{Pb}$ (II) appearing to have attracted the majority of the interest of the scientific community since it is discussed in almost $1 / 3$ of the selected articles.

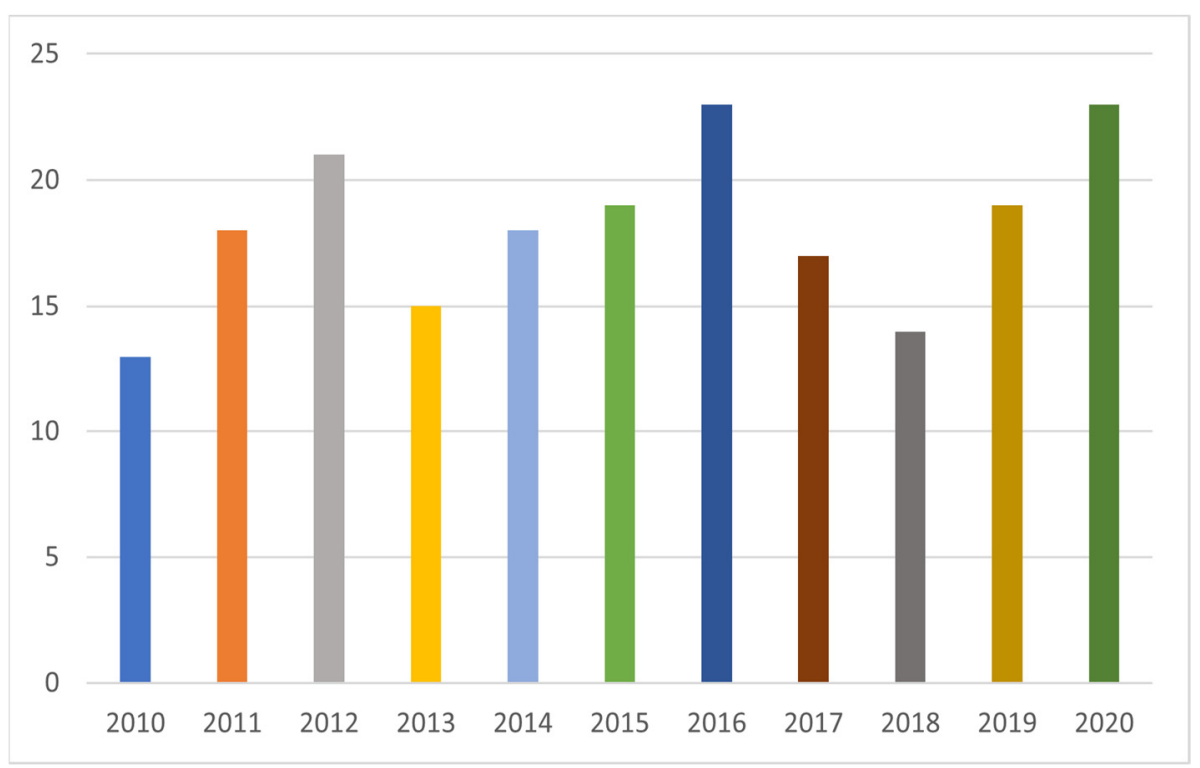

Figure 2. Selection of research articles per year. 


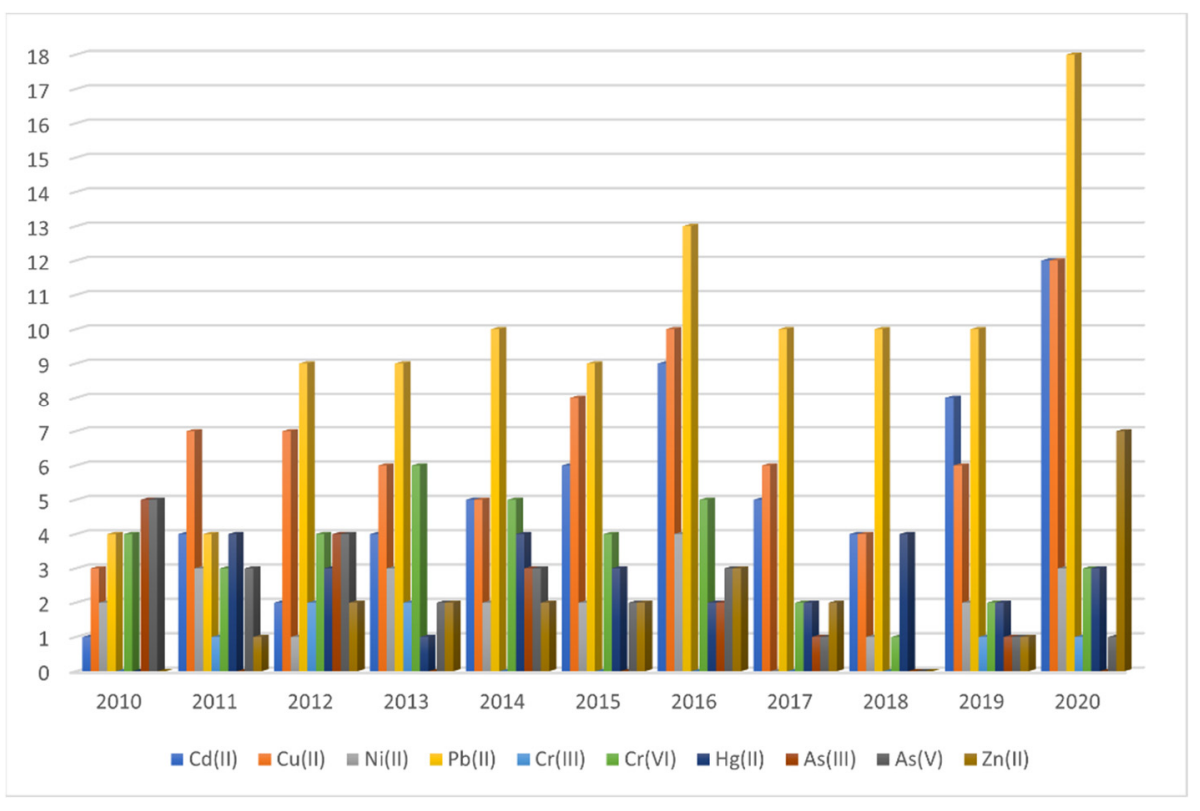

Figure 3. Research interest in each heavy metal per year since 2010.

\subsection{Synthesis and Characterization of Magnetic Nanoparticles}

The adsorption method stages are synthesis, characterization of magnetic nanoparticles, and finally, evaluation of adsorption by specific methods, which are described below. Synthesis and characterization are correlated since the results of the initial stage, which is synthesis, are visualized and analysed through characterization.

Over the years, nanoparticle synthesis has been performed by top-down or bottomup approaches with the use of chemical techniques, such as coprecipitation [70], microemulsions [71], sol-gel synthesis [72,73], sonochemical reactions [74], hydrothermal reactions [75], hydrolysis [76,77], thermolysis of precursors [78], flow injection [79,80], electrospray syntheses [81], solvothermal method [82], and laser ablation [83]

Moreover, several factors are affecting the size and the stability of nanoparticles, such as $\mathrm{pH}$ and temperature. Different sizes and shapes of nanoparticles could be synthesized by using the above synthesis methods. The average nanoparticle size of iron oxides increases with the increase in reaction temperature [84], and their size is estimated from either the Scherrer or Debye equation $[85,86]$. It is important to note that the Scherrer equation can only be applied for sizes between 100 and $200 \mathrm{~nm}[87,88]$.

The stabilization of iron oxide particles is crucial to obtain magnetic colloidal ferrofluids that are stable against aggregation in both a biological medium and a magnetic field [69]. In order to achieve stabilization, several coating methods [89,90] are used, such as monomeric stabilizers (carboxylates, phosphates) [91,92], inorganic materials (silica, gold, dextran), and polymeric stabilizers (chitosan, polyvinyl alcohol, polyethylene glycol, alginate) $[93,94]$. The functionalization of nanoparticles' surface is of major importance for tuning the overall properties of particles to fit targeted applications [95].

A crucial factor is the surface charge of metal oxides due to the presence of hydroxyl groups depending on the $\mathrm{pH}$ of the solution (i.e., the surface of the magnetite will be positive or negative), the point of zero charge ( $\mathrm{pHpzc}$ ) caused by the amphoteric behaviour of hydroxylated surface groups, and the interaction between surface sites and the electrolyte species [96]. Therefore, the surface charge as well as the surface potential is essential in predicting surface reactions, surface complex formation, ion transfer, and so forth. Zeta potential, which reflects the potential difference between moving particles and the bulk phase, is a basic and major parameter to indicate the surface surroundings of a particle [97]. Hence, at the isoelectric point (PZC) particles flocculate because of the density of the surface charge, which is very small [98]. 
In addition, the efficiency of water purification techniques is strongly dependent on the efficiency of the adsorption and/or coprecipitation processes, which in turn are strongly influenced by the zeta potential of the colloidal substrate $[99,100]$.

The ability and efficiency of the adsorption technologies in water treatment also depend on the characteristics and functions of adsorbents [101]. Another issue for magnetic nanoparticles at high temperatures is that the magnetic moments will rapidly fluctuate [102]. Generally, the saturation magnetization (MS) values found in nanostructured materials are usually smaller than the corresponding bulk phase, provided that no change in ionic configurations occurs [103]. The optimum saturated magnetization value could lead to a fast separation process, and the adsorbents could be easily separated from aqueous solution $[104,105]$.

To obtain pure synthesized materials, they are isolated with the use of a magnetic field and, in almost all cases, washed with ultrapure water several times [106] and finally dried in a vacuum oven in order to remove redundant diamines [107].

The majority of existing research background focuses on the magnetic nanoparticles of iron oxide due to their superparamagnetic behaviour, high corrosion resistance [108], and low toxicity [109]. Moreover, composites of carbon and $\mathrm{Fe}_{3} \mathrm{O}_{4}$ exhibit excellent microwave absorption but also possess good conductivity and strength [110].

Hematite, magnetite, and maghemite excel significantly among iron oxides due to their unique magnetic, catalytic, and other properties [111,112]. Zerovalent metals are effectively reductant when reacting with oxidized pollutants in water [113]. Pollutant removal by $\gamma-\mathrm{Fe}_{2} \mathrm{O}_{3}$ nanoparticles has been recognized to be resulting from electrostatic interactions. Oxygen atoms on the surface of the iron oxides $\mathrm{Fe}_{3} \mathrm{O}_{4}$ and $\gamma-\mathrm{Fe}_{2} \mathrm{O}_{3}$ can be polarized under various $\mathrm{pH}$ values. More specifically, when the $\mathrm{pH}$ value is below the point of zero charge, the surface of the iron oxides possesses positive charges, attracting negatively charged pollutants $[114,115]$. The physical and magnetic properties of iron oxides are presented in Table 3.

Table 3. Physical and magnetic properties of iron oxide [116,117].

\begin{tabular}{|c|c|c|c|}
\hline Molecular Formula & $\alpha-\mathrm{Fe}_{2} \mathrm{O}_{3}$ & $\mathrm{Fe}_{3} \mathrm{O}_{4}$ & $\gamma-\mathrm{Fe}_{2} \mathrm{O}_{3}$ \\
\hline Density $\left(\mathrm{g} / \mathrm{cm}^{3}\right)$ & 5.26 & 5.18 & 4.87 \\
\hline Melting point $\left({ }^{\circ} \mathrm{C}\right)$ & 1350 & 1583-1597 & - \\
\hline Hardness & 6.5 & 5.5 & 5 \\
\hline Type of magnetism & $\begin{array}{l}\text { Weakly ferromagnetic, } \\
\text { antiferromagnetic }\end{array}$ & Ferromagnetic & Ferromagnetic \\
\hline Curie temperature (K) & - & 850 & 948 \\
\hline $\begin{array}{l}\text { Point of zero charge } \\
(\mathrm{pHpzc})\end{array}$ & - & - & 7.5 \\
\hline Morin temperature $(\mathrm{K})$ & 263 & - & - \\
\hline Neel temperature (K) & $948<\mathrm{T}_{\mathrm{N}}<963$ & - & - \\
\hline a & $\begin{array}{c}- \\
\text { Rhombohedral }\end{array}$ & $\begin{array}{l}0.8394 \\
\text { Cubic }\end{array}$ & $\begin{array}{l}0.8346 \\
\text { Cubic }\end{array}$ \\
\hline
\end{tabular}

Hematite is the most stable form of iron oxide polymorphs [117], and nanostructures can be obtained in various morphologies [118]; hence hematite is well fitted to the needs of each application. Hematite's magnetic properties are related to its particle size: above the Neel temperature $\left(T_{N}\right)$ it is ferromagnetic, between $T_{N}$ and $T_{M}$ it is characterized by a reorientational magnetic phase transition, and below the Morin temperature $\left(T_{M}\right)$ it becomes antiferromagnetic [119].

Ferromagnetic iron oxides, such as magnetite with $\mathrm{M}_{\mathrm{s}} 92-100(\mathrm{emu} / \mathrm{g})$ and magnemite with $M_{s} 60-80(e m u / g)$, have a critical diameter value $\left(D_{c r}\right)$; below $D_{c r}$, more energy is required to create a domain wall than to support the external magnetostatic energy of a 
single domain state [120]. The magnemite and magnetite nanoparticles become supermagnetic when the sizes of the nanoparticles are under 20 [121] and $25 \mathrm{~nm}$, respectively. The above similar physical properties arise from the alike crystal structure. In general, as the size of the $\mathrm{Fe}_{3} \mathrm{O}_{4}$ nanoparticles decreases, the saturation magnetization also decreases [122]. In addition, magnetite iron oxide can be easily oxidized to $\mathrm{Fe}_{2} \mathrm{O}_{3}$ or dissolved in an acidic medium; therefore, anaerobic conditions during synthesis should be obtained in order to avoid oxidation [70]. In this context, it is obvious that plenty of factors are affecting the synthesis of magnetic nanoparticles, consequently raising the difficulty of the method. The sizes of the bare particles of hematite, magnetite, and maghemite under various synthesis methods and environments are presented in Table 4.

Table 4. Synthesis methods for bare iron oxide nanoparticles' sizes under various conditions.

\begin{tabular}{|c|c|c|c|c|c|}
\hline Method & Iron Oxide & $\begin{array}{l}\text { Nanoparticle } \\
\text { Size (nm) }\end{array}$ & $\mathrm{pH}$ & $\begin{array}{c}\text { Temperature } \\
\left({ }^{\circ} \mathrm{C}\right)\end{array}$ & Reference \\
\hline Coprecipitation & $\mathrm{Fe}_{3} \mathrm{O}_{4}$ & 8.3 & 11 & 45 & [86] \\
\hline $\begin{array}{c}\text { Sonochemical } \\
\text { coprecipitation } \\
\text { (ultrasound assistance) }\end{array}$ & $\mathrm{Fe}_{3} \mathrm{O}_{4}$ & 13 & & & [74] \\
\hline $\begin{array}{l}\text { Sonochemical } \\
\text { coprecipitation }\end{array}$ & $\mathrm{Fe}_{3} \mathrm{O}_{4}$ & 17 & & & [74] \\
\hline Coprecipitation & $\mathrm{Fe}_{3} \mathrm{O}_{4}$ & $10-20$ & 9 & 80 & [123] \\
\hline $\begin{array}{l}\text { Ultrasonic-assisted } \\
\text { chemical } \\
\text { coprecipitation }\end{array}$ & $\mathrm{Fe}_{3} \mathrm{O}_{4}$ & 15 & 7 & 60 & [124] \\
\hline Coprecipitation & $\mathrm{Fe}_{3} \mathrm{O}_{4}$ & 13.2 & 11 & 85 & [86] \\
\hline Sol-gel & $\mathrm{Fe}_{3} \mathrm{O}_{4}$ & 2.02 & & 200 & [73] \\
\hline Sol-gel & $\mathrm{Fe}_{3} \mathrm{O}_{4}$ & 5.58 & & 400 & [73] \\
\hline \multirow[t]{8}{*}{ Sol-gel } & $\mathrm{Fe}_{3} \mathrm{O}_{4}$ & 8.35 & & 600 & [73] \\
\hline & $\mathrm{Fe}_{3} \mathrm{O}_{4}$ & 23 & & & [125] \\
\hline & $\gamma-\mathrm{Fe}_{2} \mathrm{O}_{3}$ & 2 & & 130 & {$[84]$} \\
\hline & $\gamma-\mathrm{Fe}_{2} \mathrm{O}_{3}$ & 4.5 & & 180 & [84] \\
\hline & $\gamma-\mathrm{Fe}_{2} \mathrm{O}_{3}$ & 6.1 & & 200 & [84] \\
\hline & $\gamma-\mathrm{Fe}_{2} \mathrm{O}_{3}$ & 9 & & 230 & [84] \\
\hline & $\gamma-\mathrm{Fe}_{2} \mathrm{O}_{3}$ & 12 & & 250 & [84] \\
\hline & $\gamma-\mathrm{Fe}_{2} \mathrm{O}_{3}$ & 25.5 & & 250 & [125] \\
\hline Solvothermal & $\gamma-\mathrm{Fe}_{2} \mathrm{O}_{3}$ & 3000 & & 400 & [126] \\
\hline $\begin{array}{l}\text { Mechanochemical } \\
\text { milling }\end{array}$ & $\mathrm{Fe}_{2} \mathrm{O}_{3}$ & 10,000 & & & [127] \\
\hline $\begin{array}{l}\text { Mechanochemical } \\
\text { milling }\end{array}$ & $\alpha-\mathrm{Fe}_{2} \mathrm{O}_{3}$ & 17.1 & & & [128] \\
\hline $\begin{array}{l}\text { Ultrasonic spray } \\
\text { pyrolysis }\end{array}$ & $\alpha-\mathrm{Fe}_{2} \mathrm{O}_{3}$ & 18 & & 400 & [118] \\
\hline \multirow[t]{2}{*}{$\begin{array}{c}\text { Ultrasonic spray } \\
\text { pyrolysis }\end{array}$} & $\alpha-\mathrm{Fe}_{2} \mathrm{O}_{3}$ & 33 & & 600 & [118] \\
\hline & $\alpha-\mathrm{Fe}_{2} \mathrm{O}_{3}$ & 53.7 & & 500 & [125] \\
\hline
\end{tabular}

However, the use of magnetic nanoparticles, which provide larger removal capacity, higher reactivity, high surface-area-to-volume ratio, high degree of dispersion, excellent adsorption affinity, and catalytic activity [129], also presents a challenge in terms of recovery, 
stability, and toxicity. In chemical terms, iron oxide nanoparticles can be oxidized in air, resulting in the loss of dispersibility and magnetism [70].

Additionally, economic factors and the difficulty of separating them from the water phase make the use of pure iron oxide nanoparticles as an adsorbent dysfunctional [130]. Hence, surface modification could be the solution to these problems, offering protection of the magnetic core from dissolution in acid but also control over the agglomeration of magnetic nanoparticles. On the other hand, modification may have a negative impact on the activity of the particles, so modification approaches that can provide synergy between the physiochemical properties and the effectiveness of contaminant removal need further research $[61,131,132]$. In addition, low-cost absorbents with high metal-binding capacity are increasingly being utilized for the removal of heavy metals.

The characterization of nanoparticles is a major part of the method [133], for having the optimum adsorption capacity, the nanoparticles must be well defined in frames that depend on several parameters. These parameters determine and affect the nanoparticles' applicability, such as size, shape, size distribution, degree of aggregation, surface charge, and surface area [134-137]. The most common characterization method for nanoparticles of $<100 \mathrm{~nm}$ size is transmission electron microscopy (TEM), which is used for the characterization mostly of the size, shape, and dispersion of magnetic nanoparticles but also for shape heterogeneity and accumulation $[133,134,138]$. The size distribution of the nanoparticles can be measured by a dynamic laser scattering analyser (DLS) [136,138-140]. The identification of the functional groups in the synthesized magnetic nanoparticles can be obtained by Fourier-transform infrared spectroscopy (FTIR) technique. The absorption spectra of the nanoparticles were determined using ultraviolet-visible (UV-VIS) spectroscopy [141,142]. Zeta potential measurement is used for the determination of the surface charge of nanoparticles. In general, nanoparticles with zeta potential values greater than the absolute value of $30 \mathrm{mv}$ present good stability [143]. X-ray diffraction (XRD) is used for structural characterization, such as structural determination, shape, and size $[110,144]$. Magnetic force microscopy (MFM) is used for the investigation of the magnetic behaviour with high spatial resolution for static magnetic fields $[143,145,146]$.

\subsection{Adsorption Evaluation Strategies}

Characterization methods are necessary for the evaluation of the adsorption. Among the most widely employed methods are Fourier-transform infrared spectroscopy (FTIR) technique, ultraviolet-visible (UV-VIS) spectroscopy, ICP analysis, and magnetic property measurement by a VSM (vibrating sample magnetometer).

Apart from the adsorption capacity, further analysis of the adsorption process performance is obtained from the thermodynamic and kinetic aspects [147]. The adsorption method could be separated in reaction and diffusion models; for the examination of these two models, several methods have been developed.

The pollutant removal efficiency $\mathrm{R}$ and adsorption capacity $\mathrm{q}$ are calculated using Equations (1) and (2):

$$
\begin{gathered}
\mathrm{R}=\left(C_{0}-\mathrm{Ce}\right) \times 100 / C_{0} \\
\mathrm{q}=\left(C_{0}-\mathrm{C}\right) \times \mathrm{V} / \mathrm{M}
\end{gathered}
$$

where $\mathrm{C}_{0}(\mathrm{mg} / \mathrm{L})$ and $\mathrm{C}(\mathrm{mg} / \mathrm{L})$ are the initial and final pollutant concentrations in the solution, respectively. $\mathrm{V}(\mathrm{L})$ is the volume of the solution, and $\mathrm{M}(\mathrm{g})$ is the mass of the magnetic nanoparticles [148].

When an adsorbate-containing phase is contacted with the adsorbent for sufficient time, then the adsorption equilibrium is established [149].

The equilibrium correlations and performance are described by the interactions between adsorbents and pollutants, which are known as adsorption isotherms [63]. These isotherms provide plenty of information that, if utilized properly, permits the optimization of the adsorption mechanism pathways, the expression of the surface properties and capacities of adsorbents, and the effective design of the adsorption systems [150]. Sometimes it is 
difficult to estimate whether the equilibrium has been reached due to the kinetic hysteresis that occurs when a fast kinetic adsorption reaction is followed by slower adsorptions. The two parameters of the Langmuir adsorption isotherm are used for the analysis of the adsorption process according to Equation (3) [151-153]:

$$
\mathrm{q}_{\mathrm{e}}=\mathrm{q}_{\mathrm{m}} \times \mathrm{K}_{\mathrm{L}} \mathrm{C}_{\mathrm{e}} /\left(1+\mathrm{K}_{\mathrm{L}} \mathrm{C}_{\mathrm{e}}\right)
$$

where $\mathrm{q}_{\mathrm{e}}(\mathrm{mg} / \mathrm{g})$ is the equilibrium adsorption capacity of the heavy metal ions, $\mathrm{q}_{\mathrm{m}}(\mathrm{mg} / \mathrm{g})$ is the maximum adsorption capacity of the heavy metal ions, $\mathrm{C}_{\mathrm{e}}(\mathrm{mg} / \mathrm{L})$ is the equilibrium concentration of the ions, and $\mathrm{K}_{\mathrm{L}}$ is the Langmuir adsorption constant. The two-parameter isotherm Freundlich model is employed also according to Equation (4) [65,151,152]:

$$
\mathrm{q}_{\mathrm{e}}=\mathrm{K}_{\mathrm{F}} \times\left(\mathrm{C}_{\mathrm{e}}^{\frac{1}{\mathrm{n}}}\right)
$$

where $\mathrm{q}_{\mathrm{e}}(\mathrm{mg} / \mathrm{g})$ is the equilibrium adsorption capacity of the heavy metal ions, $\mathrm{q}_{\mathrm{m}}(\mathrm{mg} / \mathrm{g})$ is the maximum adsorption capacity of the heavy metal ions, $\mathrm{C}_{\mathrm{e}}(\mathrm{mg} / \mathrm{L})$ is the equilibrium concentration of the ions, $\mathrm{K}_{\mathrm{F}}$ is the Freundlich constant indicating the adsorption capacity, and $\mathrm{n}$ is the heterogeneity factor representing the adsorption intensity.

The limitations of the Freundlich isotherm model arise from the fact that it is an empirical model, its behaviour is linear only for certain concentrations, and finally, the constant $\mathrm{K}_{\mathrm{F}}$ varies with the change of temperature [154].

Another isotherm model arises from the combination of Langmuir and Freundlich expressions for the prediction of the heterogeneous adsorption systems [155], the so-called Sips model, which is described by Equation (5) [65,151,156]:

$$
\mathrm{q}_{\mathrm{e}}=\mathrm{q}_{\mathrm{s}} \times \mathrm{K}_{\mathrm{s}} \mathrm{C}_{\mathrm{e}}^{\mathrm{m}} /\left(1+\mathrm{K}_{\mathrm{s}} \mathrm{C}_{\mathrm{e}}^{\mathrm{m}}\right)
$$

where $\mathrm{q}_{\mathrm{s}}(\mathrm{mL} / \mathrm{mg})$ is the specific adsorption capacity at saturation, $\mathrm{K}_{\mathrm{s}}(\mathrm{mL} / \mathrm{mg})$ is the Sips isotherm constant, and $\mathrm{m}$ is the heterogeneity factor. The majority of the studied research works have employed the isotherm models described above.

The adsorption mechanism and the metal ion uptake rate, to establish the time dependence of the residual adsorbate in solution, are provided by the kinetic analysis of the adsorption process [157]. Several models have been employed to describe the kinetics, but the pseudo first order and the pseudo second order have dominated [158] in the existing research field for the adsorption reaction model. The linearized pseudo first order was introduced by Lagergren (1898) and is described by Equation (6) [159,160]:

$$
\ln \left(\mathrm{q}_{\mathrm{e}}-\mathrm{q}(\mathrm{t})\right)=\ln \mathrm{q}_{\mathrm{e}}-\mathrm{K}_{1} \times \mathrm{t}
$$

where $\mathrm{q}_{\mathrm{t}}$ and $\mathrm{q}_{\mathrm{e}}$ are the amounts of adsorption at time $t$ and equilibrium per unit mass of adsorbent $\mathrm{mg} / \mathrm{g}$, respectively, and $\mathrm{k}_{1}$ is the pseudo first-order rate constant $1 / \mathrm{h}$. The transformed linear pseudo second order is given by Equation (7) [159-161]:

$$
\frac{\mathrm{t}}{\mathrm{q}(\mathrm{t})}=\frac{1}{\mathrm{~K}_{2} \mathrm{q}_{\mathrm{e}}^{2}}-\frac{\mathrm{t}}{\mathrm{q}_{\mathrm{e}}}
$$

where $\mathrm{q}(\mathrm{t})$ and $\mathrm{q}_{\mathrm{e}}$ are the amount of adsorption at time $\mathrm{t}$ and equilibrium per unit mass of adsorbent $\mathrm{mg} / \mathrm{g}$, respectively, and $\mathrm{k}_{2}$ is the pseudo second-order rate constant $\mathrm{g} / \mathrm{mg} / \mathrm{h}$. The pseudo first order and the pseudo second order are empirical models, and because of that, the physical meanings cannot investigate the mass transfer mechanisms [162].

Thermodynamic analysis determines the feasibility, spontaneity, and heat change of the adsorption process [160]. The thermodynamic equations given by Van't Hoff are described by Equations (8) and (9) [159,160,163]:

$$
\Delta \mathrm{G}^{0}=-\mathrm{RT} \ln \mathrm{K}_{\mathrm{L}}
$$




$$
\Delta \mathrm{G}^{0}=\Delta \mathrm{H}^{0}-\mathrm{T} \Delta \mathrm{S}^{0}
$$

where the parameters are the free energy (Gibbs) change $\Delta \mathrm{G}^{0}$, the enthalpy change $\Delta \mathrm{H}^{0}$, and the entropy change $\Delta S^{0}$ and are estimated from the change in the thermodynamic equilibrium. Additionally, $\mathrm{R}(8.314 \mathrm{~J} / \mathrm{mol} \times \mathrm{K})$ is the gas constant, $\mathrm{T}\left({ }^{\circ} \mathrm{K}\right)$ is the absolute temperature, and $\mathrm{K}_{\mathrm{L}}(\mathrm{L} / \mathrm{mol})$ is the Langmuir constant. The exothermic reaction of the adsorption is confirmed by the negative values of the enthalpy, which also indicate decreased randomness at the solid/solution interface with some structural changes in the adsorbate and adsorbent [164].

From the above mathematical analysis of the adsorption process, it can be understood that in order to extract safe results from the experiments, the evaluation strategies should be meticulously followed. In summary, the adsorption isotherms are employed for the evaluation of the adsorption capacity and the investigation of the characteristics of adsorption, while the pseudo first- and second-order equations are used to illustrate the adsorption kinetics of the adsorbent surfaces [165].

\section{Main Findings during the Last Decade}

Restrictions on the use of bare hematite, magnetite, and maghemite nanoparticles force the majority of researchers to synthesize modified nanoparticles from iron oxide. A large number of parameters apart from those of the magnetic nanoparticle synthesis, which have been analysed above, affect the adsorption efficiency of heavy metals, among them being $\mathrm{pH}$, contact time, temperature, adsorbent dose, and initial ion concentration $[58,166]$. $\mathrm{pH}$ is a factor that is involved not only at this stage of the method but also during the synthesis of nanoparticles; almost in all cases, the $\mathrm{pH}$ values are different between these two stages. $\mathrm{pH}$ is directly related to the competition ability of hydrogen and metal ions to the adsorbent surface active sites, for by increasing the $\mathrm{pH}$ value due to the formation of soluble hydroxylated metal complexes, the metal uptake capacity decreases [161], the maximum adsorption capacity that is observed varies from $\mathrm{pH} 2$ to 9 , and most researchers achieve optimum adsorption capacity at $\mathrm{pH} 5-7$. The adsorbent dosage is directly related to the adsorbent capacity since it determines the contact areas between the adsorbent and the adsorbate [167]. Moreover, when the adsorbent dose is increasing, the number of available binding sites is also increasing; but as the equilibrium point of adsorption is reached, the efficiency does not reflect the available sites and remains steady [161]. A critical point also exists for the contact time parameter, since initially, removal rates increase rapidly with time; then they gradually decrease due to the availability of the binding sites until the equilibrium is reached [161]. Experiments show that even for the same adsorbent but for different pollutants, the ideal contact time may vary significantly, as in some cases it

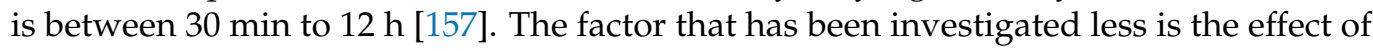
coexisting cations/anions, as in previous parameters, the initial ion concentration does not differ; thus removal rates increase with increasing initial concentrations until a point where the rates remain unaffected [161]. Research shows that these cations/anions that are contained in water have no significant influence on adsorption capacity $[168,169]$, since the adsorbent surfaces suggest multisurface adsorption active sites. Moreover, differences in the radius of heavy metal ions have significant influence on adsorption efficiency [170], ions with a smaller radius have higher mobility in aqueous solutions, and therefore, they have a lower tendency to adsorb on magnetic nanoparticles. Additionally, the temperature that is used during the adsorption process varies between 15 and $45^{\circ} \mathrm{C}$, although in some rare occasions, it reaches $70^{\circ} \mathrm{C}$.

Every research method must be evaluated according to the criteria that have been set from the beginning. At this phase of the literature review, the results of heavy metal adsorption in aquatic solutions using bare or modified magnetic iron oxide nanoparticles are summarized. In order to be able to evaluate the methods through the experimental results since 2010, many factors must be taken into consideration. Initially, many researchers used different nanoparticle compounds for various pollutants, but also observed a large selectivity of the initial parameters (i.e., adsorbent dosage, contact time, $\mathrm{pH}$, temperature), 
which creates difficulties in the categorization of the experimental results. Despite that, the main purpose of every work is the removal of heavy metals; thus adsorption capacity is the main factor that has been focused on in each research. Hence, Table 5 provides useful information, such as the time of equilibrium/contact time, $\mathrm{pH}$, adsorption capacity or removal efficiency, and temperature during the adsorption process, which could lead to safe extractions for the applicability of the method, which is based on the findings during the last decade. Additionally, the findings are listed in chronological order. Due to several nanoparticle compounds, the equilibrium time is not constant but has large dispersion.

Table 5. Adsorption capacity, removal efficiency, and system conditions.

\begin{tabular}{|c|c|c|c|c|c|c|c|c|c|}
\hline $\begin{array}{l}\text { Year of } \\
\text { Publish }\end{array}$ & $\begin{array}{c}\text { Magnetic } \\
\text { Nanoparticle }\end{array}$ & $\begin{array}{l}\text { Heavy } \\
\text { Metal } \\
\text { Ion }\end{array}$ & $\begin{array}{l}\text { Adsorption } \\
\text { Capacity } \\
\text { (mg/g) }^{1}\end{array}$ & $\begin{array}{l}\text { Removal } \\
\text { Efficiency } \\
(\%)\end{array}$ & $\begin{array}{l}\text { Time } \\
(\mathrm{min})\end{array}$ & $\mathrm{pH}$ & $\begin{array}{l}\text { Tempe- } \\
\text { rature } \\
\left({ }^{\circ} \mathrm{C}\right)\end{array}$ & $\begin{array}{c}\text { Number } \\
\text { of } \\
\text { Cycles }\end{array}$ & Ref. \\
\hline \multirow{16}{*}{2010} & $\begin{array}{c}\text { Amino- } \\
\text { functionalized } \\
\mathrm{Fe}_{3} \mathrm{O}_{4}\end{array}$ & $\mathrm{Cu}(\mathrm{II})$ & 25.77 & & 5 & 6 & 25 & 15 & [171] \\
\hline & $\mathrm{Fe}_{3} \mathrm{O}_{4}$ & $\operatorname{As}(\mathrm{V})$ & 44.1 & & & 3 & 25 & & [169] \\
\hline & $\mathrm{Fe}_{3} \mathrm{O}_{4}$ & $\mathrm{As}(\mathrm{III})$ & 49.8 & & & 7 & 25 & & [169] \\
\hline & $\mathrm{MnFe}_{2} \mathrm{O}_{4}$ & $\operatorname{As}(\mathrm{V})$ & 90.4 & & & 3 & 25 & & [169] \\
\hline & $\mathrm{MnFe}_{2} \mathrm{O}_{4}$ & As(III) & 93.8 & & & 7 & 25 & & [169] \\
\hline & $\mathrm{CoFe}_{2} \mathrm{O}_{4}$ & $\mathrm{As}(\mathrm{V})$ & 73.8 & & & 3 & 25 & & [169] \\
\hline & $\mathrm{CoFe}_{2} \mathrm{O}_{4}$ & As(III) & 100.3 & & & 7 & 25 & & [169] \\
\hline & $\begin{array}{c}\text { Amino- } \\
\text { functionalized } \\
\mathrm{Fe}_{3} \mathrm{O}_{4} @ \mathrm{SiO}_{2}\end{array}$ & $\mathrm{Cu}(\mathrm{II})$ & $\begin{array}{c}0.69 \\
\mathrm{mmol} / \mathrm{g}\end{array}$ & & & & 45 & 4 & [172] \\
\hline & $\begin{array}{c}\text { Amino- } \\
\text { functionalized } \\
\mathrm{Fe}_{3} \mathrm{O}_{4} @ \mathrm{SiO}_{2}\end{array}$ & $\mathrm{Cu}(\mathrm{II})$ & $\begin{array}{c}0.60 \\
\mathrm{mmol} / \mathrm{g}\end{array}$ & & & & 35 & 4 & [172] \\
\hline & $\begin{array}{c}\text { Amino- } \\
\text { functionalized } \\
\mathrm{Fe}_{3} \mathrm{O}_{4} @ \mathrm{SiO}_{2}\end{array}$ & $\mathrm{Cu}(\mathrm{II})$ & $\begin{array}{c}0.47 \\
\mathrm{mmol} / \mathrm{g}\end{array}$ & & & & 25 & 4 & [172] \\
\hline & $\begin{array}{c}\text { Amino- } \\
\text { functionalized } \\
\mathrm{Fe}_{3} \mathrm{O}_{4} @ \mathrm{SiO}_{2}\end{array}$ & $\mathrm{~Pb}(\mathrm{II})$ & $\begin{array}{c}0.54 \\
\mathrm{mmol} / \mathrm{g}\end{array}$ & & & & 45 & 4 & [172] \\
\hline & $\begin{array}{c}\text { Amino- } \\
\text { functionalized } \\
\mathrm{Fe}_{3} \mathrm{O}_{4} @ \mathrm{SiO}_{2}\end{array}$ & $\mathrm{~Pb}(\mathrm{II})$ & $\begin{array}{c}0.45 \\
\mathrm{mmol} / \mathrm{g}\end{array}$ & & & & 35 & 4 & [172] \\
\hline & $\begin{array}{c}\text { Amino- } \\
\text { functionalized } \\
\mathrm{Fe}_{3} \mathrm{O}_{4} @ \mathrm{SiO}_{2}\end{array}$ & $\mathrm{~Pb}(\mathrm{II})$ & $\begin{array}{c}0.37 \\
\mathrm{mmol} / \mathrm{g}\end{array}$ & & & & 25 & 4 & [172] \\
\hline & $\begin{array}{c}\text { Amino- } \\
\text { functionalized } \\
\mathrm{Fe}_{3} \mathrm{O}_{4} @ \mathrm{SiO}_{2}\end{array}$ & $\mathrm{Cd}(\mathrm{II})$ & $\begin{array}{c}0.33 \\
\mathrm{mmol} / \mathrm{g}\end{array}$ & & & & 45 & 4 & [172] \\
\hline & $\begin{array}{c}\text { Amino- } \\
\text { functionalized } \\
\mathrm{Fe}_{3} \mathrm{O}_{4} @ \mathrm{SiO}_{2}\end{array}$ & $\mathrm{Cd}(\mathrm{II})$ & $\begin{array}{c}0.27 \\
\mathrm{mmol} / \mathrm{g}\end{array}$ & & & & 35 & 4 & [172] \\
\hline & $\begin{array}{c}\text { Amino- } \\
\text { functionalized } \\
\mathrm{Fe}_{3} \mathrm{O}_{4} @ \mathrm{SiO}_{2}\end{array}$ & $\mathrm{Cd}(\mathrm{II})$ & $\begin{array}{c}0.20 \\
\mathrm{mmol} / \mathrm{g}\end{array}$ & & & & 25 & 4 & [172] \\
\hline
\end{tabular}


Table 5. Cont.

\begin{tabular}{|c|c|c|c|c|c|c|c|c|c|}
\hline $\begin{array}{l}\text { Year of } \\
\text { Publish }\end{array}$ & $\begin{array}{c}\text { Magnetic } \\
\text { Nanoparticle }\end{array}$ & $\begin{array}{l}\text { Heavy } \\
\text { Metal } \\
\text { Ion }\end{array}$ & $\begin{array}{l}\text { Adsorption } \\
\text { Capacity } \\
\text { (mg/g) }{ }^{1}\end{array}$ & $\begin{array}{l}\text { Removal } \\
\text { Efficiency } \\
\quad(\%)\end{array}$ & $\begin{array}{l}\text { Time } \\
(\mathrm{min})\end{array}$ & $\mathrm{pH}$ & $\begin{array}{l}\text { Tempe- } \\
\text { rature } \\
\left({ }^{\circ} \mathrm{C}\right)\end{array}$ & $\begin{array}{l}\text { Number } \\
\text { of } \\
\text { Cycles }\end{array}$ & Ref. \\
\hline \multirow{21}{*}{2010} & GA-APTES-NPs & $\mathrm{Cu}(\mathrm{II})$ & 61.07 & 75.3 & 15 & $4-5.3$ & $20 \pm 0.1$ & 3 & [173] \\
\hline & $\mathrm{Fe}_{3} \mathrm{O}_{4}$ & $\mathrm{~Pb}(\mathrm{II})$ & 36 & & 30 & 5.5 & & 5 & [168] \\
\hline & EDA-MPs-10 & $\mathrm{Cr}(\mathrm{VI})$ & 61.35 & & 60 & 2.5 & 35 & & [107] \\
\hline & EDA-MPs-8 & $\mathrm{Cr}(\mathrm{VI})$ & 60.98 & & 60 & 2.5 & 35 & & [107] \\
\hline & EDA-MPs-6 & $\mathrm{Cr}(\mathrm{VI})$ & 49.5 & & 60 & 2.5 & 35 & & [107] \\
\hline & EDA-MPs-4 & $\mathrm{Cr}(\mathrm{VI})$ & 36.63 & & 60 & 2.5 & 35 & & [107] \\
\hline & EDA-MPs-2 & $\mathrm{Cr}(\mathrm{VI})$ & 32.15 & & 60 & 2.5 & 35 & & [107] \\
\hline & $\mathrm{Fe}_{3} \mathrm{O}_{4}-\gamma-\mathrm{Fe}_{2} \mathrm{O}_{3}$ & $\mathrm{As}(\mathrm{III})$ & 3.69 & 96 & & 2 & & & [115] \\
\hline & $\mathrm{Fe}_{3} \mathrm{O}_{4}-\gamma-\mathrm{Fe}_{2} \mathrm{O}_{3}$ & $\mathrm{As}(\mathrm{V})$ & 3.71 & 96 & & 2 & & & [115] \\
\hline & $\mathrm{Fe}_{3} \mathrm{O}_{4}-\gamma-\mathrm{Fe}_{2} \mathrm{O}_{3}$ & $\mathrm{Cr}(\mathrm{VI})$ & 2.4 & 99 & & 2 & & & [115] \\
\hline & $\mathrm{Fe}_{3} \mathrm{O}_{4}$ & $\mathrm{~Pb}(\mathrm{II})$ & 63.33 & & & 6 & & & [174] \\
\hline & $\mathrm{Fe}_{3} \mathrm{O}_{4}$ & $\mathrm{Ni}(\mathrm{II})$ & 52.55 & & & 6 & & & [174] \\
\hline & $\mathrm{Fe}_{3} \mathrm{O}_{4} @ \mathrm{SiO}_{2}$ & $\mathrm{~Pb}(\mathrm{II})$ & & 97.34 & & 6 & & 5 & [175] \\
\hline & Nanoiron & $\mathrm{Ni}(\mathrm{II})$ & 11.53 & & & 5 & 25 & & [176] \\
\hline & EDA-NMPs & $\mathrm{Cr}(\mathrm{VI})$ & 136.98 & & 30 & 2.5 & 35 & & [177] \\
\hline & DEDA-NMPs & $\mathrm{Cr}(\mathrm{VI})$ & 149.25 & & 30 & 2.5 & 35 & & [177] \\
\hline & TETA-NMPs & $\mathrm{Cr}(\mathrm{VI})$ & 204.08 & & 30 & 2.5 & 35 & & [177] \\
\hline & TEPA-NMPs & $\mathrm{Cr}(\mathrm{VI})$ & 370.37 & & 30 & 2 & 35 & & [177] \\
\hline & Magnetite NPs & $\mathrm{Cr}(\mathrm{VI})$ & & 82 & 20 & 2 & & & [106] \\
\hline & $\mathrm{Fe}_{3} \mathrm{O}_{4}-\gamma \mathrm{Fe} 2 \mathrm{O}_{3}$ & As(III) & 4.75 & 91 & 180 & 6.5 & & & [178] \\
\hline & $\mathrm{Fe}_{3} \mathrm{O}_{4}-\gamma \mathrm{Fe}_{2} \mathrm{O}_{3}$ & $\mathrm{As}(\mathrm{V})$ & 4.85 & 92 & 180 & 6.5 & & & [178] \\
\hline \multirow{11}{*}{2011} & $\gamma-\mathrm{Fe}_{2} \mathrm{O}_{3}$ & $\operatorname{Hg}(\mathrm{II})$ & 140 & & & 8 & & & [157] \\
\hline & $\begin{array}{l}\text { Iron oxide-coated } \\
\text { perlite (IOCP) }\end{array}$ & $\mathrm{As}(\mathrm{V})$ & 0.39 & & 5 & $6.5-7$ & & & [165] \\
\hline & $\begin{array}{c}\gamma-\mathrm{Fe}_{2} \mathrm{O}_{3} \text { onto } \\
\text { ball-milled expanded } \\
\text { perlite carrier }\end{array}$ & $\operatorname{As}(\mathrm{V})$ & 4.64 & & & 7 & & & [179] \\
\hline & $\mathrm{NiFe}_{2} \mathrm{O}_{4}$ & $\mathrm{Cu}(\mathrm{II})$ & 55.83 & & & & & & [180] \\
\hline & $\mathrm{NiFe}_{2} \mathrm{O}_{4}$ & $\mathrm{Cr}(\mathrm{VI})$ & 36.95 & & & & & & [180] \\
\hline & $\mathrm{NiFe}_{2} \mathrm{O}_{4}$ & $\mathrm{Ni}(\mathrm{II})$ & 37.02 & & & & & & [180] \\
\hline & $\begin{array}{l}\text { EDTAD-treated } \\
\mathrm{Fe}_{3} \mathrm{O}_{4}\end{array}$ & $\mathrm{~Pb}(\mathrm{II})$ & 99.26 & & & 5.5 & 30 & & [181] \\
\hline & $\begin{array}{l}\text { EDTAD-treated } \\
\mathrm{Fe}_{3} \mathrm{O}_{4}\end{array}$ & $\mathrm{Cd}(\mathrm{II})$ & 48.70 & & & 6 & 30 & & [181] \\
\hline & $\mathrm{Fe}_{3} \mathrm{O}_{4} @ \mathrm{SiO}_{2}$-MIIP & $\mathrm{Cu}(\mathrm{II})$ & 24.2 & & & & & 5 & [182] \\
\hline & $\mathrm{Fe}_{3} \mathrm{O}_{4} @ \mathrm{SiO}_{2}-\mathrm{NIP}$ & $\mathrm{Cu}(\mathrm{II})$ & 5.2 & & & & & & [182] \\
\hline & $\mathrm{Fe}_{3} \mathrm{O}_{4}-\mathrm{TW}$ & $\mathrm{Ni}(\mathrm{II})$ & 38.3 & & & & & 5 & [183] \\
\hline
\end{tabular}


Table 5. Cont.

\begin{tabular}{|c|c|c|c|c|c|c|c|c|c|}
\hline $\begin{array}{l}\text { Year of } \\
\text { Publish }\end{array}$ & $\begin{array}{c}\text { Magnetic } \\
\text { Nanoparticle }\end{array}$ & $\begin{array}{l}\text { Heavy } \\
\text { Metal } \\
\text { Ion }\end{array}$ & $\begin{array}{l}\text { Adsorption } \\
\text { Capacity } \\
(\mathrm{mg} / \mathrm{g})^{1}\end{array}$ & $\begin{array}{c}\text { Removal } \\
\text { Efficiency } \\
(\%)\end{array}$ & $\begin{array}{l}\text { Time } \\
(\mathrm{min})\end{array}$ & $\mathrm{pH}$ & $\begin{array}{l}\text { Tempe- } \\
\text { rature } \\
\left({ }^{\circ} \mathrm{C}\right)\end{array}$ & $\begin{array}{c}\text { Number } \\
\text { of } \\
\text { Cycles }\end{array}$ & Ref. \\
\hline \multirow{25}{*}{2011} & $\gamma-\mathrm{Fe}_{2} \mathrm{O}_{3} @ \mathrm{Fe}_{3} \mathrm{O}_{4}$ & $\mathrm{Cr}(\mathrm{VI})$ & 74.07 & & 30 & & 35 & & [184] \\
\hline & $\gamma-\mathrm{Fe}_{2} \mathrm{O}_{3} @ \mathrm{Fe}_{3} \mathrm{O}_{4}$ & $\mathrm{Cr}(\mathrm{VI})$ & 78.13 & & 30 & & 25 & & [184] \\
\hline & $\gamma-\mathrm{Fe}_{2} \mathrm{O}_{3} @ \mathrm{Fe}_{3} \mathrm{O}_{4}$ & $\mathrm{Cr}(\mathrm{VI})$ & 83.33 & & 30 & & 15 & & [184] \\
\hline & $\begin{array}{c}\text { Polyrhodanine- } \\
\text { coated } \\
\gamma-\mathrm{Fe}_{2} \mathrm{O}_{3}\end{array}$ & $\mathrm{Hg}(\mathrm{II})$ & 179 & & & & & 5 & [157] \\
\hline & Polypyrrole $/ \mathrm{F}_{3} \mathrm{O}_{4}$ & $\mathrm{Cr}(\mathrm{VI})$ & 169.49 & & $30-180$ & 2 & 25 & 3 & [185] \\
\hline & Polypyrrole $/ \mathrm{F}_{3} \mathrm{O}_{4}$ & $\mathrm{Cr}(\mathrm{VI})$ & 204.08 & & $30-180$ & 2 & 35 & & [185] \\
\hline & Polypyrrole $/ \mathrm{F}_{3} \mathrm{O}_{4}$ & $\mathrm{Cr}(\mathrm{VI})$ & 238.09 & & 30-180 & 2 & 45 & & [185] \\
\hline & $\begin{array}{l}\text { Iron oxide-modified } \\
\text { sewage sludge }\end{array}$ & $\mathrm{Pb}(\mathrm{II})$ & 42.4 & & & 6 & $25 \pm 0.1$ & & [186] \\
\hline & $\begin{array}{l}\text { Iron oxide-modified } \\
\text { sewage sludge }\end{array}$ & $\mathrm{Cu}(\mathrm{II})$ & 17.3 & & & 6 & $25 \pm 0.1$ & & [186] \\
\hline & $\begin{array}{l}\text { Iron oxide-modified } \\
\text { sewage sludge }\end{array}$ & $\mathrm{Cd}(\mathrm{II})$ & 14.7 & & & 7 & $25 \pm 0.1$ & & [186] \\
\hline & $\begin{array}{l}\text { Iron oxide-modified } \\
\text { sewage sludge }\end{array}$ & $\mathrm{Ni}(\mathrm{II})$ & 7.8 & & & 7 & $25 \pm 0.1$ & & [186] \\
\hline & $\mathrm{SH}-\mathrm{mSi} @ \mathrm{Fe}_{3} \mathrm{O}_{4}$ & $\mathrm{Hg}(\mathrm{II})$ & 260 & & & 6.5 & 25 & 6 & [187] \\
\hline & $\mathrm{SH}-\mathrm{mSi} @ \mathrm{Fe}_{3} \mathrm{O}_{4}$ & $\mathrm{~Pb}(\mathrm{II})$ & 91.5 & & & 6.5 & 25 & 6 & [187] \\
\hline & $\mathrm{Fe}_{3} \mathrm{O}_{4} @ \mathrm{SiO}_{2}$ & $\mathrm{Hg}(\mathrm{II})$ & & 98 & & & & & [188] \\
\hline & MWCNT/IO/CD & $\mathrm{Cu}(\mathrm{II})$ & & 59 & & 5.5 & 25.15 & & [189] \\
\hline & $\begin{array}{c}\text { CS-co-MMB-co-PAA } \\
\text { hydrogel }\end{array}$ & $\mathrm{Pb}(\mathrm{II})$ & 163.90 & & & 5.5 & 25 & & [190] \\
\hline & $\begin{array}{c}\text { CS-co-MMB-co-PAA } \\
\text { hydrogel }\end{array}$ & $\mathrm{Cd}(\mathrm{II})$ & 135.51 & & & 5.5 & 25 & & [190] \\
\hline & $\begin{array}{c}\text { CS-co-MMB-co-PAA } \\
\text { hydrogel }\end{array}$ & $\mathrm{Cu}(\mathrm{II})$ & 152.42 & & & 5.5 & 25 & & [190] \\
\hline & $\begin{array}{l}\text { MWCNT/nano-iron } \\
\text { oxide }\end{array}$ & $\mathrm{Cr}(\mathrm{III})$ & & 82 & & 5 & & & [191] \\
\hline & $\begin{array}{l}\text { MWCNT/nano-iron } \\
\text { oxide }\end{array}$ & $\mathrm{Cr}(\mathrm{III})$ & & 88 & & 6 & & & [191] \\
\hline & Nano- $\mathrm{Fe}_{3} \mathrm{O}_{4}$ & $\mathrm{Cu}(\mathrm{II})$ & 8.90 & & & 5 & 25 & & [192] \\
\hline & $\begin{array}{c}\text { PEI-grafted magnetic } \\
\text { porous }\end{array}$ & $\mathrm{Cu}(\mathrm{II})$ & 157.8 & & 10 & $6-7.5$ & & 4 & [193] \\
\hline & $\begin{array}{c}\text { PEI-grafted magnetic } \\
\text { porous }\end{array}$ & $\mathrm{Zn}(\mathrm{II})$ & 138.8 & & 10 & $6-7.5$ & & 4 & [193] \\
\hline & $\begin{array}{c}\text { PEI-grafted magnetic } \\
\text { porous }\end{array}$ & $\mathrm{Cd}(\mathrm{II})$ & 105.2 & & 10 & $6-7.5$ & & 4 & [193] \\
\hline & $\begin{array}{c}\mathrm{Fe}_{3} \mathrm{O}_{4} \text {-coated boron } \\
\text { nitride nanotubes }\end{array}$ & $\mathrm{As}(\mathrm{V})$ & 0.96 & & 720 & 9 & 25 & 4 & [194] \\
\hline
\end{tabular}


Table 5. Cont.

\begin{tabular}{|c|c|c|c|c|c|c|c|c|c|}
\hline $\begin{array}{l}\text { Year of } \\
\text { Publish }\end{array}$ & $\begin{array}{c}\text { Magnetic } \\
\text { Nanoparticle }\end{array}$ & $\begin{array}{l}\text { Heavy } \\
\text { Metal } \\
\text { Ion }\end{array}$ & $\begin{array}{l}\text { Adsorption } \\
\text { Capacity } \\
(\mathrm{mg} / \mathrm{g})^{1}\end{array}$ & $\begin{array}{l}\text { Removal } \\
\text { Efficiency } \\
(\%)\end{array}$ & $\begin{array}{l}\text { Time } \\
(\min )\end{array}$ & $\mathrm{pH}$ & $\begin{array}{l}\text { Tempe- } \\
\text { rature } \\
\left({ }^{\circ} \mathrm{C}\right)\end{array}$ & $\begin{array}{l}\text { Number } \\
\text { of } \\
\text { Cycles }\end{array}$ & Ref. \\
\hline \multirow{28}{*}{2012} & $\mathrm{Fe}_{3} \mathrm{O}_{4}-\mathrm{C}$ & $\mathrm{Pb}(\mathrm{II})$ & 126 & & & 6 & & & [195] \\
\hline & $\begin{array}{l}\text { Pectin-coated iron } \\
\text { oxide }\end{array}$ & $\mathrm{Cu}(\mathrm{II})$ & 48.9 & & & & & & [196] \\
\hline & $\mathrm{Fe}_{3} \mathrm{O}_{4} @ \mathrm{ZrO}_{2}$ & $\mathrm{Cr}(\mathrm{III})$ & 24.5 & & & $8-9$ & & & [131] \\
\hline & $\begin{array}{l}\text { MNPs-Ca-alginate } \\
\text { immobilized } \\
\text { P. chrysosporium }\end{array}$ & $\mathrm{Pb}(\mathrm{II})$ & 176.33 & & & & 35 & 5 & [197] \\
\hline & $\mathrm{AF}-\mathrm{Fe}_{3} \mathrm{O}_{4}$ & $\mathrm{Cu}(\mathrm{II})$ & 523.6 & & 120 & 7 & & & [198] \\
\hline & $\mathrm{AF}-\mathrm{Fe}_{3} \mathrm{O}_{4}$ & $\mathrm{Cd}(\mathrm{II})$ & 446.4 & & 120 & 7 & & & [198] \\
\hline & $\mathrm{AF}-\mathrm{Fe}_{3} \mathrm{O}_{4}$ & $\mathrm{~Pb}(\mathrm{II})$ & 369.0 & & 120 & 7 & & & [198] \\
\hline & $\begin{array}{c}\mathrm{Fe}_{3} \mathrm{O}_{4} @ \mathrm{APS} @ \mathrm{AA}-\mathrm{CA}- \\
\text { CA }\end{array}$ & $\mathrm{Cd}(\mathrm{II})$ & 29.6 & & 45 & 5.5 & 25 & 4 & [199] \\
\hline & $\begin{array}{c}\mathrm{Fe}_{3} \mathrm{O}_{4} @ \mathrm{APS} @ \mathrm{AA}-\mathrm{CA}- \\
\text { CA }\end{array}$ & $\mathrm{Zn}(\mathrm{II})$ & 43.4 & & 45 & 5.5 & 25 & 4 & [199] \\
\hline & $\begin{array}{c}\mathrm{Fe}_{3} \mathrm{O}_{4} @ \mathrm{APS} @ \mathrm{AA}-\mathrm{co}- \\
\text { CA }\end{array}$ & $\mathrm{Pb}(\mathrm{II})$ & 166.1 & & 45 & 5.5 & 25 & 4 & [199] \\
\hline & $\begin{array}{c}\mathrm{Fe}_{3} \mathrm{O}_{4} @ \mathrm{APS} @ \mathrm{AA}-\mathrm{CA}- \\
\text { CA }\end{array}$ & $\mathrm{Cu}(\mathrm{II})$ & 126.9 & & 45 & 5.5 & 25 & 4 & [199] \\
\hline & $\mathrm{Fe}_{3} \mathrm{O}_{4}-\mathrm{SiO}_{2}$-poly & As(III) & $84 \pm 5$ & & 120 & 6 & 30 & & [104] \\
\hline & $\mathrm{Fe}_{3} \mathrm{O}_{4}-\mathrm{SiO}_{2}$-poly & $\mathrm{Cu}(\mathrm{II})$ & $65 \pm 3$ & & 120 & 6 & 30 & & [104] \\
\hline & $\mathrm{Fe}_{3} \mathrm{O}_{4}-\mathrm{SiO}_{2}$-poly & $\mathrm{Cr}(\mathrm{III})$ & $77 \pm 3$ & & 120 & 5.3 & 30 & & [104] \\
\hline & Acid-coated $\mathrm{Fe}_{3} \mathrm{O}_{4}$ & $\mathrm{As}(\mathrm{V})$ & 16.56 & & & & & & [200] \\
\hline & Acid-coated $\mathrm{Fe}_{3} \mathrm{O}_{4}$ & $\mathrm{As}(\mathrm{III})$ & 46.06 & & & & & & [200] \\
\hline & $\begin{array}{c}\gamma-\mathrm{Fe}_{2} \mathrm{O}_{3} \\
\text { functionalized with } \\
\text { citrate ions }\end{array}$ & $\mathrm{Ni}(\mathrm{II})$ & $\begin{array}{c}0.57 \\
\mathrm{mmol} / \mathrm{g}\end{array}$ & & 15 & $\begin{array}{l}6 \pm \\
0.5\end{array}$ & & & [201] \\
\hline & $\mathrm{Fe}_{3} \mathrm{O}_{4} @ \mathrm{CTAB}$ & $\operatorname{As}(\mathrm{V})$ & 23.07 & & 2 & 6 & & 5 & [202] \\
\hline & $\mathrm{Fe}_{3} \mathrm{O}_{4}-\gamma-\mathrm{Fe}_{2} \mathrm{O}_{3}$ & $\mathrm{Cr}(\mathrm{VI})$ & 6 & & & 4 & 10 & & [202] \\
\hline & $\mathrm{Fe}_{3} \mathrm{O}_{4}-\gamma-\mathrm{Fe}_{2} \mathrm{O}_{3}$ & $\mathrm{Cr}(\mathrm{VI})$ & 6.9 & & & 4 & 22 & & [202] \\
\hline & $\mathrm{Fe}_{3} \mathrm{O}_{4}-\gamma-\mathrm{Fe}_{2} \mathrm{O}_{3}$ & $\mathrm{Cr}(\mathrm{VI})$ & 7 & & & 4 & 50 & & [202] \\
\hline & $\mathrm{Fe}_{3} \mathrm{O}_{4}-\mathrm{PEI} 800-\mathrm{MMT}$ & $\mathrm{Cr}(\mathrm{VI})$ & 8.77 & & & & & & [203] \\
\hline & $\begin{array}{c}\mathrm{Fe}_{3} \mathrm{O}_{4}-\mathrm{PEI} 25000- \\
\text { MMT }\end{array}$ & $\mathrm{Cr}(\mathrm{VI})$ & 7.69 & & & & & & [203] \\
\hline & MPTS-CNTs $/ \mathrm{Fe}_{3} \mathrm{O}_{4}$ & $\mathrm{Hg}$ (II) & 65.52 & & & 6.5 & $25 \pm 0.2$ & & [204] \\
\hline & MPTS-CNTs $/ \mathrm{Fe}_{3} \mathrm{O}_{4}$ & $\mathrm{~Pb}(\mathrm{II})$ & 65.40 & & & 6.5 & $25 \pm 0.2$ & & [204] \\
\hline & $\mathrm{rGO}-\mathrm{Fe}(0)-\mathrm{Fe}_{3} \mathrm{O}_{4}$ & $\mathrm{As}(\mathrm{III})$ & 44 & & 60 & 7 & 25 & & [205] \\
\hline & $\mathrm{rGO}-\mathrm{Fe}_{3} \mathrm{O}_{4}$ & $\mathrm{As}(\mathrm{III})$ & 21 & & 60 & 7 & 25 & & [205] \\
\hline & $\begin{array}{l}\text { Nanomagnetite } \\
\text { (NMT) }\end{array}$ & $\mathrm{Cu}(\mathrm{II})$ & 14.3 & & 70 & & 45 & & [206] \\
\hline
\end{tabular}


Table 5. Cont.

\begin{tabular}{|c|c|c|c|c|c|c|c|c|c|}
\hline $\begin{array}{l}\text { Year of } \\
\text { Publish }\end{array}$ & $\begin{array}{c}\text { Magnetic } \\
\text { Nanoparticle }\end{array}$ & $\begin{array}{c}\text { Heavy } \\
\text { Metal } \\
\text { Ion }\end{array}$ & $\begin{array}{c}\text { Adsorption } \\
\text { Capacity } \\
(\mathrm{mg} / \mathrm{g})^{1}\end{array}$ & $\begin{array}{c}\text { Removal } \\
\text { Efficiency } \\
(\%)\end{array}$ & $\begin{array}{l}\text { Time } \\
(\min )\end{array}$ & $\mathrm{pH}$ & $\begin{array}{l}\text { Tempe- } \\
\text { rature } \\
\left({ }^{\circ} \mathrm{C}\right)\end{array}$ & $\begin{array}{c}\text { Number } \\
\text { of } \\
\text { Cycles }\end{array}$ & Ref. \\
\hline \multirow{13}{*}{2012} & $\begin{array}{c}\text { Nanomagnetite } \\
\text { (NMT) }\end{array}$ & $\mathrm{As}(\mathrm{V})$ & 6.5 & & 120 & & 45 & & [206] \\
\hline & $\begin{array}{c}\text { Water-soluble } \mathrm{Fe}_{3} \mathrm{O}_{4} \\
\text { nanoparticles }\end{array}$ & $\mathrm{Pb}(\mathrm{II})$ & 96.8 & & 60 & 7 & 18 & & [207] \\
\hline & $\begin{array}{c}\text { Water-soluble } \mathrm{Fe}_{3} \mathrm{O}_{4} \\
\text { nanoparticles }\end{array}$ & $\mathrm{Cr}(\mathrm{VI})$ & 41.5 & & 90 & 7 & 18 & & [207] \\
\hline & TF-SCMNPs & $\mathrm{Hg}(\mathrm{II})$ & 207 & 93.76 & 15 & 6 & 22.5 & & [208] \\
\hline & M-MIONPs & $\mathrm{Hg}(\mathrm{II})$ & & 98.6 & 4 & 9 & 25 & & [209] \\
\hline & $\mathrm{Fe}_{3} \mathrm{O}_{4}-\mathrm{RGO}-\mathrm{MnO}_{2}$ & As(III) & 14.04 & & & 7 & 25 & & [210] \\
\hline & $\mathrm{Fe}_{3} \mathrm{O}_{4}-\mathrm{RGO}-\mathrm{MnO}_{2}$ & $\mathrm{As}(\mathrm{V})$ & 12.22 & & & 7 & 25 & & [210] \\
\hline & $\mathrm{MWCNTs} / \mathrm{Fe}_{3} \mathrm{O}_{4}$ & $\mathrm{~Pb}(\mathrm{II})$ & 41.77 & & & 5.3 & & 5 & [211] \\
\hline & $\begin{array}{c}\mathrm{MWCNTs} / \mathrm{Fe}_{3} \mathrm{O}_{4^{-}} \\
\mathrm{NH}_{2}\end{array}$ & $\mathrm{~Pb}(\mathrm{II})$ & 75.02 & & & 5.3 & & 5 & [211] \\
\hline & $\gamma-\mathrm{Fe}_{2} \mathrm{O}_{3}$ & $\mathrm{Cu}(\mathrm{II})$ & 71.42 & & & $\begin{array}{l}6 \pm \\
0.1 \\
\end{array}$ & $25 \pm 1$ & & [212] \\
\hline & $\gamma-\mathrm{Fe}_{2} \mathrm{O}_{3}$ & $\mathrm{Zn}(\mathrm{II})$ & 111.11 & & & $\begin{array}{l}6 \pm \\
0.1 \\
\end{array}$ & $25 \pm 1$ & & [212] \\
\hline & $\gamma-\mathrm{Fe}_{2} \mathrm{O}_{3}$ & $\mathrm{~Pb}(\mathrm{II})$ & 84.95 & & & $\begin{array}{c}6 \pm \\
0.1\end{array}$ & $25 \pm 1$ & & [212] \\
\hline & $\mathrm{Fe}_{3} \mathrm{O}_{4} / \mathrm{CS} / \mathrm{PAA}$ & $\mathrm{Cu}(\mathrm{II})$ & 193 & & & & & & [213] \\
\hline \multirow{13}{*}{2013} & $\mathrm{Fe}_{3} \mathrm{O}_{4} / \mathrm{SiO}_{2}$ & $\mathrm{~Pb}(\mathrm{II})$ & 14.65 & & & 4 & 27 & & [214] \\
\hline & $\mathrm{Fe}_{3} \mathrm{O}_{4} / \mathrm{SiO}_{2}$ & $\mathrm{~Pb}(\mathrm{II})$ & 16.83 & & & 4 & 50 & & [214] \\
\hline & $\mathrm{Fe}_{3} \mathrm{O}_{4} / \mathrm{SiO}_{2}$ & $\mathrm{~Pb}(\mathrm{II})$ & 17.65 & & & 4 & 70 & & [214] \\
\hline & $\mathrm{Fe}_{2} \mathrm{O}_{3}-\mathrm{Al} 2 \mathrm{O}_{3}$ & $\mathrm{Cu}(\mathrm{II})$ & 4.98 & & 60 & 6 & & 4 & [215] \\
\hline & $\mathrm{Fe}_{2} \mathrm{O}_{3}-\mathrm{Al}_{2} \mathrm{O}_{3}$ & $\mathrm{~Pb}(\mathrm{II})$ & 23.75 & & 60 & 6 & & 4 & [215] \\
\hline & $\mathrm{Fe}_{2} \mathrm{O}_{3}-\mathrm{Al}_{2} \mathrm{O}_{3}$ & $\mathrm{Ni}(\mathrm{II})$ & 32.36 & & 60 & 6 & & 4 & [215] \\
\hline & $\mathrm{Fe}_{2} \mathrm{O}_{3}-\mathrm{Al}_{2} \mathrm{O}_{3}$ & $\mathrm{Hg}(\mathrm{II})$ & 63.69 & & 60 & 6 & & 4 & [215] \\
\hline & $\begin{array}{l}\text { Mixed magnetite- } \\
\text { hematite }\end{array}$ & $\mathrm{Pb}(\mathrm{II})$ & 617.3 & & 60 & 7 & 25 & & [216] \\
\hline & $\begin{array}{l}\text { Mixed magnetite- } \\
\text { hematite }\end{array}$ & $\mathrm{Cr}(\mathrm{III})$ & 277.0 & & 120 & 7 & 25 & & [216] \\
\hline & $\begin{array}{l}\text { Mixed magnetite-- } \\
\text { hematite }\end{array}$ & $\mathrm{Cd}(\mathrm{II})$ & 223.7 & & 1440 & 7 & 25 & & [216] \\
\hline & $\begin{array}{c}\text { Chitosan-coated } \\
\mathrm{MnFe}_{2} \mathrm{O}_{4} \text { (CCMNPs) }\end{array}$ & $\mathrm{Cu}(\mathrm{II})$ & 22.6 & & & 6 & & 5 & [217] \\
\hline & $\begin{array}{c}\text { Chitosan-coated } \\
\mathrm{MnFe}_{2} \mathrm{O}_{4} \text { (CCMNPs) }\end{array}$ & $\mathrm{Cr}(\mathrm{VI})$ & 15.4 & & & 6 & & 5 & [217] \\
\hline & $\gamma$-PGA $/ \mathrm{Fe}_{3} \mathrm{O}_{4} \mathrm{MNPs}$ & $\mathrm{Cr}(\mathrm{III})$ & 162.6 & 99.66 & 120 & 6 & 30 & & [218] \\
\hline
\end{tabular}


Table 5. Cont.

\begin{tabular}{|c|c|c|c|c|c|c|c|c|c|}
\hline $\begin{array}{l}\text { Year of } \\
\text { Publish }\end{array}$ & $\begin{array}{c}\text { Magnetic } \\
\text { Nanoparticle }\end{array}$ & $\begin{array}{c}\text { Heavy } \\
\text { Metal } \\
\text { Ion }\end{array}$ & $\begin{array}{c}\text { Adsorption } \\
\text { Capacity } \\
(\mathrm{mg} / \mathrm{g})^{1}\end{array}$ & $\begin{array}{c}\text { Removal } \\
\text { Efficiency } \\
(\%)\end{array}$ & $\begin{array}{l}\text { Time } \\
\text { (min) }\end{array}$ & $\mathrm{pH}$ & $\begin{array}{l}\text { Tempe- } \\
\text { rature } \\
\left({ }^{\circ} \mathrm{C}\right)\end{array}$ & $\begin{array}{c}\text { Number } \\
\text { of } \\
\text { Cycles }\end{array}$ & Ref. \\
\hline \multirow{24}{*}{2013} & CDpoly-MNPs & $\mathrm{Pb}(\mathrm{II})$ & 64.5 & & 45 & $5.5-6$ & 25 & 4 & [219] \\
\hline & CDpoly-MNPs & $\mathrm{Cd}(\mathrm{II})$ & 27.7 & & 45 & $5.5-6$ & 25 & & [219] \\
\hline & CDpoly-MNPs & $\mathrm{Ni}(\mathrm{II})$ & 13.2 & & 45 & $5.5-6$ & 25 & & [219] \\
\hline & $\begin{array}{c}\text { 3D flowerlike } \\
\text { a- } \mathrm{Fe}_{2} \mathrm{O}_{3}\end{array}$ & $\operatorname{As}(\mathrm{V})$ & 41.46 & & 120 & & & & [220] \\
\hline & $\begin{array}{l}\text { 3D flowerlike } \\
\text { a- } \mathrm{Fe}_{2} \mathrm{O}_{3}\end{array}$ & $\mathrm{Cr}(\mathrm{VI})$ & 33.82 & & 120 & & & & [220] \\
\hline & Magnetite nanorods & $\mathrm{Pb}(\mathrm{II})$ & 112.86 & & 60 & $5-6$ & 25 & & [221] \\
\hline & Magnetite nanorods & $\mathrm{Zn}(\mathrm{II})$ & 107.27 & & 60 & $5-6$ & 25 & & [221] \\
\hline & Magnetite nanorods & $\mathrm{Ni}(\mathrm{II})$ & 95.42 & & 60 & $5-6$ & 25 & & [221] \\
\hline & Magnetite nanorods & $\mathrm{Cd}(\mathrm{II})$ & 88.39 & & 60 & $5-6$ & 25 & & [221] \\
\hline & Magnetite nanorods & $\mathrm{Cu}(\mathrm{II})$ & 76.10 & & 60 & $5-6$ & 25 & & [221] \\
\hline & $\begin{array}{l}\text { Maghemite } \\
\text { nanotubes }\end{array}$ & $\mathrm{Pb}(\mathrm{II})$ & 71.42 & & & & & & [221] \\
\hline & $\begin{array}{l}\text { Maghemite } \\
\text { nanotubes }\end{array}$ & $\mathrm{Zn}(\mathrm{II})$ & 86.95 & & & & & & [221] \\
\hline & $\begin{array}{l}\text { Maghemite } \\
\text { nanotubes }\end{array}$ & $\mathrm{Cu}(\mathrm{II})$ & 111.11 & & & & & & [221] \\
\hline & $\begin{array}{c}\text { EDTA-modified } \\
\text { chitosan } / \mathrm{SiO}_{2} / \mathrm{Fe}_{3} \mathrm{O}_{4}\end{array}$ & $\mathrm{Cu}(\mathrm{II})$ & $\begin{array}{c}0.495 \\
\mathrm{mmol} / \mathrm{g}\end{array}$ & & 720 & 5 & 25 & 12 & [222] \\
\hline & $\begin{array}{c}\text { EDTA-modified } \\
\text { chitosan } / \mathrm{SiO}_{2} / \mathrm{Fe}_{3} \mathrm{O}_{4}\end{array}$ & $\mathrm{~Pb}(\mathrm{II})$ & $\begin{array}{c}0.045 \\
\mathrm{mmol} / \mathrm{g}\end{array}$ & & 720 & 5 & 25 & 12 & [222] \\
\hline & $\begin{array}{c}\text { EDTA-modified } \\
\text { chitosan } / \mathrm{SiO}_{2} / \mathrm{Fe}_{3} \mathrm{O}_{4}\end{array}$ & $\mathrm{Cd}(\mathrm{II})$ & $\begin{array}{c}0.040 \\
\mathrm{mmol} / \mathrm{g}\end{array}$ & & 720 & 5 & 25 & 12 & [222] \\
\hline & $\begin{array}{c}\mathrm{Fe}_{3} \mathrm{O}_{4} @ \text { mesoporous } \\
\mathrm{SiO}_{2} \text { core-shell }\end{array}$ & $\mathrm{Pb}(\mathrm{II})$ & 128.21 & & & & & 6 & [223] \\
\hline & $\begin{array}{c}\mathrm{Fe}_{3} \mathrm{O}_{4} @ \text { mesoporous } \\
\mathrm{SiO}_{2} \text { core-shell }\end{array}$ & $\mathrm{Cu}(\mathrm{II})$ & 51.81 & & & & & 6 & [223] \\
\hline & $\mathrm{Fe}_{3} \mathrm{O}_{4} / \mathrm{GO}$ & $\mathrm{Cr}(\mathrm{VI})$ & 32.33 & & & 4.5 & 20 & & [224] \\
\hline & $\begin{array}{l}\text { Hollow nestlike } \\
\alpha-\mathrm{Fe}_{2} \mathrm{O}_{3} \text { spheres }\end{array}$ & $\mathrm{As}(\mathrm{V})$ & 75.3 & 88 & 120 & & & & [225] \\
\hline & $\begin{array}{l}\text { Hollow nestlike } \\
\alpha-\mathrm{Fe}_{2} \mathrm{O}_{3} \text { spheres }\end{array}$ & $\mathrm{Cr}(\mathrm{VI})$ & 58.6 & 67 & 120 & & & & [225] \\
\hline & $\alpha-\mathrm{Fe}_{2} \mathrm{O}_{3}$ nanofibers & $\mathrm{Cr}(\mathrm{VI})$ & 16.17 & & & & 25 & 4 & [226] \\
\hline & $\mathrm{Fe}_{3} \mathrm{O}_{4} @ \mathrm{SiO}_{2}-\mathrm{NH}_{2}$ & $\mathrm{~Pb}(\mathrm{II})$ & 243.9 & & 180 & 5.2 & 25 & 5 & [227] \\
\hline & $\begin{array}{c}\text { Cyanex-301-coated } \\
\text { SPION }\end{array}$ & $\mathrm{Cr}(\mathrm{VI})$ & 30.8 & & & 2 & 23 & & [228] \\
\hline
\end{tabular}


Table 5. Cont.

\begin{tabular}{|c|c|c|c|c|c|c|c|c|c|}
\hline $\begin{array}{l}\text { Year of } \\
\text { Publish }\end{array}$ & $\begin{array}{c}\text { Magnetic } \\
\text { Nanoparticle }\end{array}$ & $\begin{array}{c}\text { Heavy } \\
\text { Metal } \\
\text { Ion }\end{array}$ & $\begin{array}{l}\text { Adsorption } \\
\text { Capacity } \\
(\mathrm{mg} / \mathrm{g})^{1}\end{array}$ & $\begin{array}{c}\text { Removal } \\
\text { Efficiency } \\
(\%)\end{array}$ & $\begin{array}{l}\text { Time } \\
(\mathrm{min})\end{array}$ & $\mathrm{pH}$ & $\begin{array}{l}\text { Tempe- } \\
\text { rature } \\
\left({ }^{\circ} \mathrm{C}\right)\end{array}$ & $\begin{array}{l}\text { Number } \\
\text { of } \\
\text { Cycles }\end{array}$ & Ref. \\
\hline \multirow{28}{*}{2014} & S-doped $\mathrm{Fe}_{3} \mathrm{O}_{4} @ \mathrm{C}$ & $\mathrm{Cu}(\mathrm{II})$ & 54.7 & & & 5 & 35 & 4 & [229] \\
\hline & $\mathrm{Fe}_{3} \mathrm{O}_{4} @ \mathrm{SiO}_{2}-\mathrm{QTPA}$ & $\mathrm{Cd}(\mathrm{II})$ & & 95.29 & 720 & & 25 & & [230] \\
\hline & $\mathrm{Fe}_{3} \mathrm{O}_{4} @ \mathrm{SiO}_{2}$-QTPA & $\mathrm{Zn}(\mathrm{II})$ & & 92.37 & 720 & & 25 & & [230] \\
\hline & $\mathrm{Fe}_{3} \mathrm{O}_{4} @ \mathrm{SiO}_{2}$-QTPA & $\mathrm{Cu}(\mathrm{II})$ & & 91.06 & 720 & & 25 & & [230] \\
\hline & $\begin{array}{c}\text { Glycine- } \\
\text { functionalized } \\
\text { maghemite } \\
\text { nanoparticles }\end{array}$ & $\mathrm{Cu}(\mathrm{II})$ & 625 & & & 6.5 & 25 & & [231] \\
\hline & $\begin{array}{l}\text { Maghemite } \\
\left(\gamma-\mathrm{Fe}_{2} \mathrm{O}_{3}\right)\end{array}$ & $\mathrm{Pb}(\mathrm{II})$ & 10.55 & & & 7.5 & 45 & & [232] \\
\hline & $\begin{array}{l}\text { Maghemite } \\
\left(\gamma-\mathrm{Fe}_{2} \mathrm{O}_{3}\right)\end{array}$ & $\mathrm{Zn}(\mathrm{II})$ & 4.79 & & & 7.5 & 45 & & [232] \\
\hline & $\begin{array}{l}\text { Maghemite } \\
\left(\gamma-\mathrm{Fe}_{2} \mathrm{O}_{3}\right)\end{array}$ & $\mathrm{Cd}(\mathrm{II})$ & 1.75 & & & 7.5 & 45 & & [232] \\
\hline & $\alpha-\mathrm{Fe}_{2} \mathrm{O}_{3}$ & $\mathrm{Cr}(\mathrm{VI})$ & 17 & & 300 & & & & [233] \\
\hline & $\begin{array}{l}\text { 3-MPA-coated } \\
\text { SPION }\end{array}$ & $\mathrm{Cr}(\mathrm{VI})$ & 45 & & & 1 & 25 & & [234] \\
\hline & $\mathrm{EDA}-\mathrm{Fe}_{3} \mathrm{O}_{4} \mathrm{NPs}$ & $\mathrm{Cr}(\mathrm{VI})$ & & 98 & 120 & 2 & & 6 & [235] \\
\hline & M-FeHT & $\mathrm{As}(\mathrm{V})$ & 1.2813 & & 15 & 9 & 25 & & [236] \\
\hline & M-FeHT & As(III) & 0.1213 & & 15 & 9 & 25 & & [236] \\
\hline & $\mathrm{Fe}_{3} \mathrm{O}_{4}$-GS & $\mathrm{Cr}(\mathrm{VI})$ & 17.29 & & 240 & $1-3.5$ & & 5 & [237] \\
\hline & $\mathrm{Fe}_{3} \mathrm{O}_{4}$-GS & $\mathrm{Pb}(\mathrm{II})$ & 27.95 & & 120 & $6-7$ & & 5 & [237] \\
\hline & $\mathrm{Fe}_{3} \mathrm{O}_{4}$-GS & $\mathrm{Hg}(\mathrm{II})$ & 23.03 & & 120 & $6-7$ & & & [237] \\
\hline & $\mathrm{Fe}_{3} \mathrm{O}_{4}$-GS & $\mathrm{Cd}(\mathrm{II})$ & 27.83 & & 120 & $6-7$ & & & [237] \\
\hline & $\mathrm{Fe}_{3} \mathrm{O}_{4}$-GS & $\mathrm{Ni}(\mathrm{II})$ & 22.07 & & 120 & $6-7$ & & & [237] \\
\hline & $\mathrm{Fe}_{3} \mathrm{O}_{4} @ \mathrm{CPS}$ & $\mathrm{Cu}(\mathrm{II})$ & 53.6 & & & 5 & 25 & 3 & [238] \\
\hline & $\mathrm{Fe}_{3} \mathrm{O}_{4} @ \mathrm{CPS}$ & $\mathrm{Cd}(\mathrm{II})$ & 87.1 & & & 6 & 25 & 3 & [238] \\
\hline & $\mathrm{Fe}_{3} \mathrm{O}_{4} @ \mathrm{CPS}$ & $\mathrm{Pb}(\mathrm{II})$ & 25.2 & & & 6 & 25 & 3 & [238] \\
\hline & $\begin{array}{c}\mathrm{MWCNT} \\
\mathrm{CoFe}_{2} \mathrm{O}_{4}-\mathrm{NH}_{2}\end{array}$ & $\mathrm{~Pb}(\mathrm{II})$ & 140.1 & & & 6 & & 5 & [239] \\
\hline & $\mathrm{Fe}_{3} \mathrm{O}_{4} /$ talc & $\mathrm{Cu}(\mathrm{II})$ & & 72.15 & & & & & [240] \\
\hline & $\mathrm{Fe}_{3} \mathrm{O}_{4} /$ talc & $\mathrm{Ni}(\mathrm{II})$ & & 50.23 & & & & & [240] \\
\hline & $\mathrm{Fe}_{3} \mathrm{O}_{4} /$ talc & $\mathrm{Pb}(\mathrm{II})$ & & 91.35 & & & & & [240] \\
\hline & $\begin{array}{c}\text { Magnetite } \\
\text { nanoparticles }\end{array}$ & $\mathrm{Cr}(\mathrm{VI})$ & 121.9 & & 60 & 5.5 & & & [241] \\
\hline & $\mathrm{CoFe}_{2} \mathrm{O}_{4}$-rGO & $\mathrm{Pb}(\mathrm{II})$ & 299.4 & & 80 & 5.3 & 25 & & [242] \\
\hline & $\mathrm{CoFe}_{2} \mathrm{O}_{4}$-rGO & $\mathrm{Pb}(\mathrm{II})$ & 274.7 & & & & 35 & & [242] \\
\hline
\end{tabular}


Table 5. Cont.

\begin{tabular}{|c|c|c|c|c|c|c|c|c|c|}
\hline $\begin{array}{l}\text { Year of } \\
\text { Publish }\end{array}$ & $\begin{array}{c}\text { Magnetic } \\
\text { Nanoparticle }\end{array}$ & $\begin{array}{l}\text { Heavy } \\
\text { Metal } \\
\text { Ion }\end{array}$ & $\begin{array}{l}\text { Adsorption } \\
\text { Capacity } \\
(\mathrm{mg} / \mathrm{g})^{1}\end{array}$ & $\begin{array}{c}\text { Removal } \\
\text { Efficiency } \\
(\%)\end{array}$ & $\begin{array}{l}\text { Time } \\
\text { (min) }\end{array}$ & $\mathrm{pH}$ & $\begin{array}{l}\text { Tempe- } \\
\text { rature } \\
\left({ }^{\circ} \mathrm{C}\right)\end{array}$ & $\begin{array}{c}\text { Number } \\
\text { of } \\
\text { Cycles }\end{array}$ & Ref. \\
\hline \multirow{15}{*}{2014} & $\mathrm{CoFe}_{2} \mathrm{O}_{4}$-rGO & $\mathrm{Pb}(\mathrm{II})$ & 253.2 & & & & 45 & & [242] \\
\hline & $\mathrm{CoFe}_{2} \mathrm{O}_{4}-\mathrm{rGO}$ & $\operatorname{Hg}(\mathrm{II})$ & 157.9 & & 60 & 4.6 & 25 & & [242] \\
\hline & $\mathrm{CoFe}_{2} \mathrm{O}_{4}-\mathrm{rGO}$ & $\operatorname{Hg}(\mathrm{II})$ & 105.1 & & & & 35 & & [242] \\
\hline & $\mathrm{CoFe}_{2} \mathrm{O}_{4}-\mathrm{rGO}$ & $\operatorname{Hg}(\mathrm{II})$ & 90.49 & & & & 45 & & [242] \\
\hline & $\begin{array}{c}\text { Graphene } \\
\text { Oxide- }-\mathrm{MnFe}_{2} \mathrm{O}_{4}\end{array}$ & $\mathrm{~Pb}(\mathrm{II})$ & 673 & & & 5 & & & [243] \\
\hline & $\mathrm{MnFe}_{2} \mathrm{O}_{4}$ & $\mathrm{~Pb}(\mathrm{II})$ & 488 & & & 5 & 60 & & [243] \\
\hline & $\begin{array}{c}\text { Graphene } \\
\text { Oxide- }-\mathrm{MnFe}_{2} \mathrm{O}_{4}\end{array}$ & As(III) & 146 & & & 6.5 & 60 & & [243] \\
\hline & $\mathrm{MnFe}_{2} \mathrm{O}_{4}$ & As(III) & 97 & & & 6.5 & 60 & & [243] \\
\hline & $\begin{array}{c}\text { Graphene } \\
\text { Oxide- }-\mathrm{MnFe}_{2} \mathrm{O}_{4}\end{array}$ & $\mathrm{As}(\mathrm{V})$ & 207 & & & 4 & 60 & & [243] \\
\hline & $\mathrm{MnFe}_{2} \mathrm{O}_{4}$ & $\mathrm{As}(\mathrm{V})$ & 136 & & & 4 & 60 & & [243] \\
\hline & Water-soluble $\mathrm{Fe}_{3} \mathrm{O}_{4}$ & $\mathrm{Hg}(\mathrm{II})$ & & $>99$ & 10 & 7 & 25 & 3 & [244] \\
\hline & $\mathrm{Fe}_{3} \mathrm{O}_{4} @ \mathrm{C}$ & $\mathrm{Pb}(\mathrm{II})$ & 90.7 & 96.3 & 10 & & & & [245] \\
\hline & $\mathrm{Fe}_{3} \mathrm{O}_{4} @ \mathrm{C}$ & $\operatorname{Hg}(\mathrm{II})$ & 83.1 & 98.1 & 10 & & & & [245] \\
\hline & $\mathrm{Fe}_{3} \mathrm{O}_{4} @ \mathrm{C}$ & $\mathrm{Cd}(\mathrm{II})$ & 39.7 & 93.8 & 10 & & & & [245] \\
\hline & $\begin{array}{l}\mathrm{Fe}_{3} \mathrm{O}_{4} @ \text { silica } \\
\text { xanthan gum }\end{array}$ & $\mathrm{Pb}(\mathrm{II})$ & 21.32 & & & 6 & 20 & 22 & [246] \\
\hline \multirow{7}{*}{2015} & $\mathrm{Fe}_{3} \mathrm{O}_{4}$-NTA & $\mathrm{Cu}(\mathrm{II})$ & 40.24 & & 35 & 5 & & & [160] \\
\hline & $\begin{array}{c}\text { Magnetic } \\
\text { chitosan/cellulose } \\
\text { hybrid microspheres } \\
\text { by } \\
\text { embedding } \gamma-\mathrm{Fe}_{2} \mathrm{O}_{3}\end{array}$ & $\mathrm{Cu}(\mathrm{II})$ & 88.21 & & & & 30 & & [247] \\
\hline & $\begin{array}{c}\text { Magnetic } \\
\text { chitosan/cellulose } \\
\text { hybrid microspheres } \\
\text { by } \\
\text { embedding } \gamma-\mathrm{Fe}_{2} \mathrm{O}_{3}\end{array}$ & $\mathrm{Cd}(\mathrm{II})$ & 61.1 & & & & 30 & & [247] \\
\hline & $\begin{array}{c}\text { Magnetic } \\
\text { chitosan/cellulose } \\
\text { hybrid microspheres } \\
\text { by } \\
\text { embedding } \gamma-\mathrm{Fe}_{2} \mathrm{O}_{3}\end{array}$ & $\mathrm{~Pb}(\mathrm{II})$ & 45.86 & & & & 30 & & [247] \\
\hline & $\begin{array}{c}\text { Mesoporous } \\
\mathrm{CoFe}_{2} \mathrm{O}_{4}\end{array}$ & $\mathrm{~Pb}(\mathrm{II})$ & 32.11 & & 480 & 5 & & & [248] \\
\hline & MAMNPs & $\mathrm{Cd}(\mathrm{II})$ & 91.5 & & 100 & 6 & & 3 & [249] \\
\hline & MAMNPs & $\mathrm{Hg}(\mathrm{II})$ & 237.6 & & 100 & 6 & & 3 & [249] \\
\hline
\end{tabular}


Table 5. Cont.

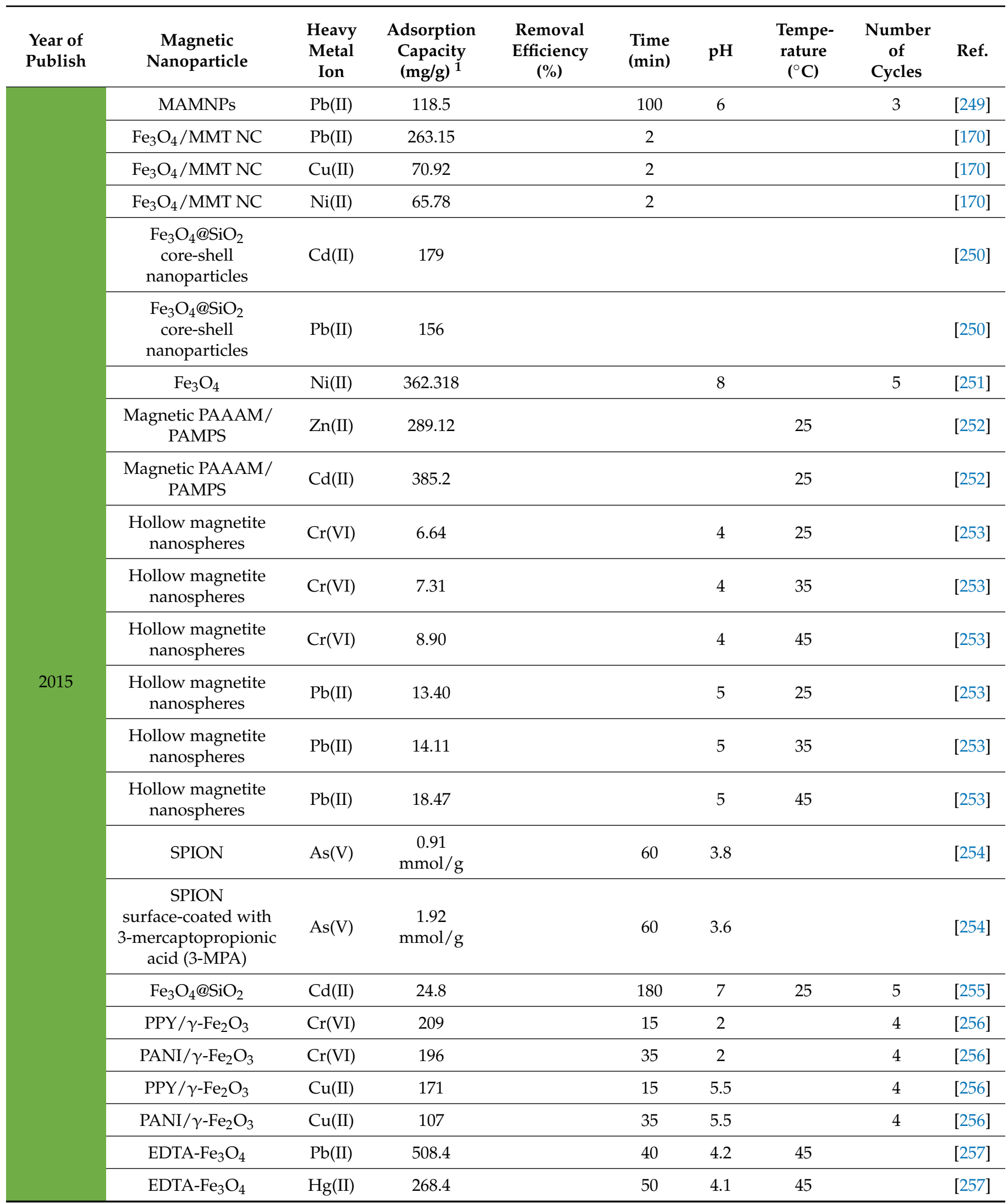


Table 5. Cont.

\begin{tabular}{|c|c|c|c|c|c|c|c|c|c|}
\hline $\begin{array}{l}\text { Year of } \\
\text { Publish }\end{array}$ & $\begin{array}{c}\text { Magnetic } \\
\text { Nanoparticle }\end{array}$ & $\begin{array}{c}\text { Heavy } \\
\text { Metal } \\
\text { Ion }\end{array}$ & $\begin{array}{l}\text { Adsorption } \\
\text { Capacity } \\
(\mathrm{mg} / \mathrm{g})^{1}\end{array}$ & $\begin{array}{c}\text { Removal } \\
\text { Efficiency } \\
(\%)\end{array}$ & $\begin{array}{l}\text { Time } \\
\text { (min) }\end{array}$ & $\mathrm{pH}$ & $\begin{array}{l}\text { Tempe- } \\
\text { rature } \\
\left({ }^{\circ} \mathrm{C}\right)\end{array}$ & $\begin{array}{c}\text { Number } \\
\text { of } \\
\text { Cycles }\end{array}$ & Ref. \\
\hline \multirow{21}{*}{2015} & EDTA- $\mathrm{Fe}_{3} \mathrm{O}_{4}$ & $\mathrm{Cu}(\mathrm{II})$ & 301.2 & & 90 & 5.1 & 45 & & [257] \\
\hline & EDTA-Fe ${ }_{3} \mathrm{O}_{4}$ & $\mathrm{~Pb}(\mathrm{II})$ & 548.1 & & & & 35 & & [257] \\
\hline & EDTA-Fe ${ }_{3} \mathrm{O}_{4}$ & $\mathrm{Hg}(\mathrm{II})$ & 242.2 & & & & 35 & & [257] \\
\hline & EDTA-Fe ${ }_{3} \mathrm{O}_{4}$ & $\mathrm{Cu}(\mathrm{II})$ & 289.4 & & & & 35 & & [257] \\
\hline & EDTA-Fe ${ }_{3} \mathrm{O}_{4}$ & $\mathrm{~Pb}(\mathrm{II})$ & 481.2 & & & & 25 & 5 & [257] \\
\hline & EDTA-Fe ${ }_{3} \mathrm{O}_{4}$ & $\mathrm{Hg}(\mathrm{II})$ & 203.1 & & & & 25 & 5 & [257] \\
\hline & EDTA- $\mathrm{Fe}_{3} \mathrm{O}_{4}$ & $\mathrm{Cu}(\mathrm{II})$ & 246.1 & & & & 25 & 5 & [257] \\
\hline & $\mathrm{Fe}_{3} \mathrm{O}_{4} @ \mathrm{SiO}_{2} /$ Schiff & $\mathrm{Cu}(\mathrm{II})$ & 97.2 & & 60 & 5 & & & [258] \\
\hline & $\mathrm{Fe}_{3} \mathrm{O}_{4} @ \mathrm{SiO}_{2} /$ Schiff & $\mathrm{Zn}(\mathrm{II})$ & 81.6 & & 60 & 5 & & & [258] \\
\hline & $\begin{array}{l}\text { PMMA-gft- } \\
\mathrm{Alg} / \mathrm{Fe}_{3} \mathrm{O}_{4}\end{array}$ & $\mathrm{~Pb}(\mathrm{II})$ & 62.5 & & & 5 & 50 & & [259] \\
\hline & $\begin{array}{l}\text { PMMA-gft- } \\
\mathrm{Alg} / \mathrm{Fe}_{3} \mathrm{O}_{4}\end{array}$ & $\mathrm{Cu}(\mathrm{II})$ & 35.71 & & & 5 & 50 & & [259] \\
\hline & SH-HMSMCS & $\mathrm{Hg}(\mathrm{II})$ & & 95 & & 6.2 & & 5 & [260] \\
\hline & $\mathrm{Ppy}-\mathrm{Fe}_{3} \mathrm{O}_{4} / \mathrm{rGO}$ & $\mathrm{Cr}(\mathrm{VI})$ & 293.3 & & & 3 & 45 & & [261] \\
\hline & $\mathrm{Ppy}-\mathrm{Fe}_{3} \mathrm{O}_{4} / \mathrm{rGO}$ & $\mathrm{Cr}(\mathrm{VI})$ & 226.8 & & & 3 & 30 & & [261] \\
\hline & $\mathrm{Ppy}-\mathrm{Fe}_{3} \mathrm{O}_{4} / \mathrm{rGO}$ & $\mathrm{Cr}(\mathrm{VI})$ & 180.8 & & & 3 & 20 & & [261] \\
\hline & $\mathrm{Co}_{0 \cdot 6} \mathrm{Fe}_{2 \cdot 4} \mathrm{O}_{4}$ & $\mathrm{~Pb}(\mathrm{II})$ & 80.32 & & 30 & & 45.18 & & [262] \\
\hline & $\mathrm{Co}_{0 \cdot 6} \mathrm{Fe}_{2 \cdot 4} \mathrm{O}_{4}$ & $\mathrm{~Pb}(\mathrm{II})$ & 70.22 & & & & 35.15 & & [262] \\
\hline & $\mathrm{Co}_{0 \cdot 6} \mathrm{Fe}_{2 \cdot 4} \mathrm{O}_{4}$ & $\mathrm{~Pb}(\mathrm{II})$ & 44.58 & & & & 25.15 & & [262] \\
\hline & $\mathrm{Fe}_{3} \mathrm{O}_{4} / \mathrm{Mg}-\mathrm{Al}-\mathrm{CO}_{3}$ & $\mathrm{Cd}(\mathrm{II})$ & 54.7 & & & & 50 & & [263] \\
\hline & $\mathrm{Fe}_{3} \mathrm{O}_{4} / \mathrm{Mg}-\mathrm{Al}-\mathrm{CO}_{3}$ & $\mathrm{Cd}(\mathrm{II})$ & 50.5 & & & & 40 & & [263] \\
\hline & $\mathrm{Fe}_{3} \mathrm{O}_{4} / \mathrm{Mg}-\mathrm{Al}-\mathrm{CO}_{3}$ & $\mathrm{Cd}(\mathrm{II})$ & 45.6 & & & & 30 & & [263] \\
\hline \multirow{10}{*}{2016} & NMag-CS & $\mathrm{Cu}(\mathrm{II})$ & 123.4 & & 120 & 5.5 & & & [264] \\
\hline & NMag-CS & $\mathrm{Pb}(\mathrm{II})$ & 114.9 & & 120 & 5.5 & & & [264] \\
\hline & NMag-CS & $\mathrm{Cr}(\mathrm{VI})$ & 116.2 & & 120 & 5.5 & & & [264] \\
\hline & NMag-CS & $\mathrm{Cd}(\mathrm{II})$ & 112.3 & & 120 & 5.5 & & & [264] \\
\hline & NMag-CS & $\mathrm{Ni}(\mathrm{II})$ & 109.8 & & 120 & 5.5 & & & [264] \\
\hline & $\begin{array}{c}\text { Amino- } \\
\text { functionalized } \\
\mathrm{Fe}_{3} \mathrm{O}_{4} @ \mathrm{SiO}_{2}\end{array}$ & $\mathrm{Zn}(\mathrm{II})$ & 169.5 & & 120 & $\begin{array}{c}5 \pm \\
0.1\end{array}$ & 25 & & [265] \\
\hline & $\mathrm{Fe}_{3} \mathrm{O}_{4}$ & $\mathrm{Cu}(\mathrm{II})$ & 37.04 & & & $4-6$ & & & [266] \\
\hline & $\mathrm{Fe}_{3} \mathrm{O}_{4}$ & $\mathrm{~Pb}(\mathrm{II})$ & 166.67 & & & $4-6$ & & & [266] \\
\hline & $\mathrm{Fe}_{2} \mathrm{O}_{3}$ & $\mathrm{Cu}(\mathrm{II})$ & 19.61 & & & $5-6$ & & 4 & [266] \\
\hline & $\mathrm{Fe}_{2} \mathrm{O}_{3}$ & $\mathrm{~Pb}(\mathrm{II})$ & 47.62 & & & $3-4$ & & 4 & [266] \\
\hline
\end{tabular}


Table 5. Cont.

\begin{tabular}{|c|c|c|c|c|c|c|c|c|c|}
\hline $\begin{array}{l}\text { Year of } \\
\text { Publish }\end{array}$ & $\begin{array}{c}\text { Magnetic } \\
\text { Nanoparticle }\end{array}$ & $\begin{array}{l}\text { Heavy } \\
\text { Metal } \\
\text { Ion }\end{array}$ & $\begin{array}{l}\text { Adsorption } \\
\text { Capacity } \\
(\mathrm{mg} / \mathrm{g})^{1}\end{array}$ & $\begin{array}{l}\text { Removal } \\
\text { Efficiency } \\
\quad(\%)\end{array}$ & $\begin{array}{l}\text { Time } \\
(\mathrm{min})\end{array}$ & $\mathrm{pH}$ & $\begin{array}{l}\text { Tempe- } \\
\text { rature } \\
\left({ }^{\circ} \mathrm{C}\right)\end{array}$ & $\begin{array}{l}\text { Number } \\
\text { of } \\
\text { Cycles }\end{array}$ & Ref. \\
\hline \multirow{31}{*}{2016} & $\mathrm{Fe}_{2} \mathrm{O}_{3} @ \mathrm{SiO}_{2}$ & As(III) & 77.7 & & 180 & 7 & 30 & & [267] \\
\hline & $\mathrm{Fe}_{2} \mathrm{O}_{3} @ \mathrm{TiO}_{2}$ & $\mathrm{As}(\mathrm{V})$ & 99.5 & & 180 & 6 & 30 & & [267] \\
\hline & $\mathrm{Fe}_{3} \mathrm{O}_{4} @ \mathrm{SiO}_{2}-\mathrm{SH}$ & $\mathrm{Hg}(\mathrm{II})$ & 132 & & & 6 & 25 & 5 & [268] \\
\hline & $\mathrm{Fe}_{3} \mathrm{O}_{4-} \mathrm{Aspa}$ & $\mathrm{Ni}(\mathrm{II})$ & 87.183 & & 70 & 6 & 40 & & [269] \\
\hline & $\mathrm{Fe}_{3} \mathrm{O}_{4}$-Aspa & $\mathrm{Ni}(\mathrm{II})$ & 58.582 & & 70 & 6 & 35 & & [269] \\
\hline & $\mathrm{Fe}_{3} \mathrm{O}_{4}$-Aspa & $\mathrm{Ni}(\mathrm{II})$ & 34.602 & & 70 & 6 & 30 & 6 & [269] \\
\hline & Henna- $\mathrm{Fe}_{3} \mathrm{O}_{4}$ & $\mathrm{Cu}(\mathrm{II})$ & 28.90 & & 20 & 5.2 & & & [270] \\
\hline & $\mathrm{Fe}_{3} \mathrm{O}_{4} /$ Bent-2.0 & $\mathrm{Pb}(\mathrm{II})$ & 81.5 & & 30 & & & & [271] \\
\hline & $\mathrm{Fe}_{3} \mathrm{O}_{4} /$ Bent-2.0 & $\mathrm{Cd}(\mathrm{II})$ & 21.7 & & 30 & & & & [271] \\
\hline & $\mathrm{Fe}_{3} \mathrm{O}_{4} /$ Bent-2.0 & $\mathrm{Cu}(\mathrm{II})$ & 19.6 & & 30 & & & & [271] \\
\hline & $\mathrm{Fe}_{3} \mathrm{O}_{4}$ & $\mathrm{~Pb}(\mathrm{II})$ & & 100 & 30 & 5 & 25 & & [272] \\
\hline & $\mathrm{Fe}_{3} \mathrm{O}_{4}$ & $\mathrm{Cr}(\mathrm{VI})$ & 34.9 & & & 2 & 45 & & [273] \\
\hline & $\mathrm{Fe}_{3} \mathrm{O}_{4}$ & $\mathrm{~Pb}(\mathrm{II})$ & 53.1 & & & 5 & 45 & & [273] \\
\hline & $\mathrm{Fe}_{3} \mathrm{O}_{4}$ & $\mathrm{Cr}(\mathrm{VI})$ & 26.8 & & & 2 & 35 & & [273] \\
\hline & $\mathrm{Fe}_{3} \mathrm{O}_{4}$ & $\mathrm{~Pb}(\mathrm{II})$ & 52.8 & & & 5 & 35 & & [273] \\
\hline & $\mathrm{Fe}_{3} \mathrm{O}_{4}$ & $\mathrm{Cr}(\mathrm{VI})$ & 20.2 & & & 2 & 25 & 2 & [273] \\
\hline & $\mathrm{Fe}_{3} \mathrm{O}_{4}$ & $\mathrm{~Pb}(\mathrm{II})$ & 52.9 & & & 5 & 25 & 2 & [273] \\
\hline & $\mathrm{a}-\mathrm{Fe}_{2} \mathrm{O}_{3}$ & $\mathrm{Cd}(\mathrm{II})$ & 127.23 & & & 6 & 20 & & [274] \\
\hline & $\mathrm{a}-\mathrm{Fe}_{2} \mathrm{O}_{3}$ & $\mathrm{Cd}(\mathrm{II})$ & 146.41 & & & 6 & 30 & & [274] \\
\hline & $\mathrm{a}-\mathrm{Fe}_{2} \mathrm{O}_{3}$ & $\mathrm{Cd}(\mathrm{II})$ & 158.48 & & & 6 & 40 & & [274] \\
\hline & $\mathrm{GO} / \mathrm{Fe}_{3} \mathrm{O}_{4}$ & $\mathrm{~Pb}(\mathrm{II})$ & 65.96 & & 180 & 3 & 20 & & [275] \\
\hline & $\mathrm{GO} / \mathrm{Fe}_{3} \mathrm{O}_{4} / \mathrm{LA}$ & $\mathrm{Pb}(\mathrm{II})$ & 53.06 & & 180 & 3 & 20 & & [275] \\
\hline & $\mathrm{GO} / \mathrm{Fe}_{3} \mathrm{O}_{4} / \mathrm{LA} / \mathrm{EDTA}$ & $\mathrm{Pb}(\mathrm{II})$ & 161.80 & & 180 & 3 & 20 & & [275] \\
\hline & $\begin{array}{c}\text { Amine- } \\
\text { functionalized } \\
\mathrm{Fe}_{3} \mathrm{O}_{4} \\
\end{array}$ & $\mathrm{Cu}(\mathrm{II})$ & & 85 & 30 & 7 & & & [276] \\
\hline & $\mathrm{Fe}_{3} \mathrm{O}_{4} @ \mathrm{SiO}_{2} @ \mathrm{TiO}_{2}$ & $\mathrm{Cu}(\mathrm{II})$ & 125 & & 30 & & & & [277] \\
\hline & $\mathrm{Fe}_{3} \mathrm{O}_{4} @ \mathrm{SiO}_{2} @ \mathrm{TiO}_{2}$ & $\mathrm{Zn}(\mathrm{II})$ & 137 & & 30 & & & & [277] \\
\hline & $\mathrm{Fe}_{3} \mathrm{O}_{4} @ \mathrm{SiO}_{2} @ \mathrm{TiO}_{2}$ & $\mathrm{Cd}(\mathrm{II})$ & 148 & & 30 & & & & [277] \\
\hline & $\mathrm{Fe}_{3} \mathrm{O}_{4} @ \mathrm{SiO}_{2} @ \mathrm{TiO}_{2}$ & $\mathrm{~Pb}(\mathrm{II})$ & 160 & & 30 & & & & [277] \\
\hline & $\begin{array}{c}\text { Rice straw } / \mathrm{Fe}_{3} \mathrm{O}_{4} \\
\text { NCs }\end{array}$ & $\mathrm{Pb}(\mathrm{II})$ & & 91.18 & & & & & [278] \\
\hline & $\begin{array}{c}\text { Rice straw } / \mathrm{Fe}_{3} \mathrm{O}_{4} \\
\text { NCs }\end{array}$ & $\mathrm{Cu}(\mathrm{II})$ & & 75.54 & & & & & [278] \\
\hline & $\mathrm{Cys}-\mathrm{Fe}_{3} \mathrm{O}_{4}$ & $\mathrm{~Pb}(\mathrm{II})$ & 183.5 & & 120 & 6 & & 5 & [279] \\
\hline
\end{tabular}


Table 5. Cont.

\begin{tabular}{|c|c|c|c|c|c|c|c|c|c|}
\hline $\begin{array}{l}\text { Year of } \\
\text { Publish }\end{array}$ & $\begin{array}{c}\text { Magnetic } \\
\text { Nanoparticle }\end{array}$ & $\begin{array}{c}\text { Heavy } \\
\text { Metal } \\
\text { Ion }\end{array}$ & $\begin{array}{l}\text { Adsorption } \\
\text { Capacity } \\
(\mathrm{mg} / \mathrm{g})^{1}\end{array}$ & $\begin{array}{l}\text { Removal } \\
\text { Efficiency } \\
(\%)\end{array}$ & $\begin{array}{l}\text { Time } \\
(\min )\end{array}$ & $\mathrm{pH}$ & $\begin{array}{l}\text { Tempe- } \\
\text { rature } \\
\left({ }^{\circ} \mathrm{C}\right)\end{array}$ & $\begin{array}{c}\text { Number } \\
\text { of } \\
\text { Cycles }\end{array}$ & Ref. \\
\hline \multirow{22}{*}{2016} & Cys- $\mathrm{Fe}_{3} \mathrm{O}_{4}$ & $\mathrm{Cd}(\mathrm{II})$ & 64.35 & & 120 & 6 & & 5 & [279] \\
\hline & $\begin{array}{c}\text { Nano-Fe }{ }_{3} \mathrm{O}_{4} @ \text { Nano- } \\
\mathrm{SiO}_{2}\end{array}$ & $\mathrm{~Pb}(\mathrm{II})$ & $\begin{array}{c}1100 \\
\mu \mathrm{mol} / \mathrm{g}\end{array}$ & & & & & & [280] \\
\hline & $\begin{array}{c}\text { Nano- } \mathrm{Fe}_{3} \mathrm{O}_{4} @ \text { Nano- } \\
\mathrm{SiO}_{2}\end{array}$ & $\mathrm{Cu}(\mathrm{II})$ & $300 \mu \mathrm{mol} / \mathrm{g}$ & & & & & & [280] \\
\hline & $\begin{array}{c}\text { Nano- } \mathrm{Fe}_{3} \mathrm{O}_{4} @ \text { Nano- } \\
\mathrm{SiO}_{2}\end{array}$ & $\mathrm{Cd}(\mathrm{II})$ & $150 \mu \mathrm{mol} / \mathrm{g}$ & & & & & & [280] \\
\hline & $\begin{array}{c}\text { Nano- } \mathrm{Fe}_{3} \mathrm{O}_{4} @ \text { Nano- } \\
\mathrm{SiO}_{2}\end{array}$ & $\mathrm{Hg}(\mathrm{II})$ & $100 \mu \mathrm{mol} / \mathrm{g}$ & & & & & & [280] \\
\hline & $\mathrm{a}-\mathrm{Fe}_{2} \mathrm{O}_{3}$ & $\mathrm{As}(\mathrm{V})$ & 38.48 & & & & 20 & & [281] \\
\hline & $\mathrm{Fe}_{3} \mathrm{O}_{4}-\mathrm{MnO}_{2}$ & $\mathrm{~Pb}(\mathrm{II})$ & 208.17 & & & & 25 & & [282] \\
\hline & $\mathrm{Fe}_{3} \mathrm{O}_{4}-\mathrm{MnO}_{2}$ & $\mathrm{Cu}(\mathrm{II})$ & 111.90 & & & & 25 & & [282] \\
\hline & $\mathrm{Fe}_{3} \mathrm{O}_{4}-\mathrm{MnO}_{2}$ & $\mathrm{Cd}(\mathrm{II})$ & 169.90 & & & & 25 & & [282] \\
\hline & $\mathrm{Fe}_{3} \mathrm{O}_{4}-\mathrm{MnO}_{2}$ & $\mathrm{Zn}(\mathrm{II})$ & 100.24 & & & & 25 & & [282] \\
\hline & $\mathrm{Fe}_{3} \mathrm{O}_{4}-\mathrm{MnO}_{2}$ & $\mathrm{Ni}(\mathrm{II})$ & 55.63 & & & & 25 & & [282] \\
\hline & $\mathrm{Fe}_{3} \mathrm{O}_{4} @ \mathrm{SiO}_{2}$-EDTA & $\mathrm{Cu}(\mathrm{II})$ & 37.59 & & 10 & 5.3 & 30 & & [283] \\
\hline & $\mathrm{Fe}_{3} \mathrm{O}_{4} @ \mathrm{SiO}_{2}$-EDTA & $\mathrm{Pb}(\mathrm{II})$ & 114.94 & & 10 & 5.3 & 30 & & [283] \\
\hline & $\mathrm{Fe}_{3} \mathrm{O}_{4} @ \mathrm{SiO}_{2}$-EDTA & $\mathrm{Ni}(\mathrm{II})$ & 32.15 & & 10 & 5.3 & 30 & & [283] \\
\hline & $\mathrm{Fe}_{3} \mathrm{O}_{4} @ \mathrm{SiO}_{2}$-EDTA & $\mathrm{Cd}(\mathrm{II})$ & 50.25 & & 10 & 5.3 & 30 & & [283] \\
\hline & $\mathrm{Fe}_{3} \mathrm{O}_{4}-\mathrm{FeB}$ & $\mathrm{Cr}(\mathrm{VI})$ & 38.9 & & & 6.3 & & & [284] \\
\hline & $\mathrm{Fe}_{3} \mathrm{O}_{4}-\mathrm{TiO}_{2}$ & $\mathrm{As}(\mathrm{V})$ & $11.434 \mu \mathrm{g} / \mathrm{g}$ & & 40 & 6.3 & 25 & & [285] \\
\hline & $\mathrm{Fe}_{3} \mathrm{O}_{4}-\mathrm{TiO}_{2}$ & As(III) & & 93 & & 11 & 25 & & [285] \\
\hline & $\begin{array}{c}\text { Chitosan } \\
\text { MWCNT/ } / \mathrm{Fe}_{3} \mathrm{O}_{4}\end{array}$ & $\mathrm{Cr}(\mathrm{VI})$ & 360.1 & & 30 & 2 & 45 & & [286] \\
\hline & $\begin{array}{c}\text { Chitosan } \\
\text { MWCNT } / \mathrm{Fe}_{3} \mathrm{O}_{4}\end{array}$ & $\mathrm{Cr}(\mathrm{VI})$ & 348.2 & & & & 35 & & [286] \\
\hline & $\begin{array}{c}\text { Chitosan } \\
\text { MWCNT } / \mathrm{Fe}_{3} \mathrm{O}_{4}\end{array}$ & $\mathrm{Cr}(\mathrm{VI})$ & 335.6 & & & & 25 & & [286] \\
\hline & AMGO & $\mathrm{Cr}(\mathrm{VI})$ & 123.4 & & & 2 & & 5 & [287] \\
\hline \multirow{5}{*}{2017} & $\mathrm{Fe}_{3} \mathrm{O}_{4} @ \mathrm{MnO}_{2}$ & $\mathrm{~Pb}(\mathrm{II})$ & 666.67 & & 120 & & 25 & & [148] \\
\hline & $\mathrm{Fe}_{3} \mathrm{O}_{4}-\mathrm{SO}_{3} \mathrm{H}$ & $\mathrm{Pb}(\mathrm{II})$ & 108.93 & & & 7 & 25 & & [288] \\
\hline & $\mathrm{Fe}_{3} \mathrm{O}_{4}-\mathrm{SO}_{3} \mathrm{H}$ & $\mathrm{Cd}(\mathrm{II})$ & 80.9 & & & 7 & 25 & & [288] \\
\hline & $\begin{array}{c}\text { PGMA-MAn } \\
\text { Copolymer@ } \mathrm{Fe}_{3} \mathrm{O}_{4}\end{array}$ & $\mathrm{~Pb}(\mathrm{II})$ & 53.33 & & 20 & 5 & 25 & & [289] \\
\hline & $\begin{array}{c}\text { PGMA-MAn } \\
\text { Copolymer@Fe } \mathrm{O}_{4}\end{array}$ & $\mathrm{Cu}(\mathrm{II})$ & 48.53 & & & 5 & & & [289] \\
\hline
\end{tabular}


Table 5. Cont.

\begin{tabular}{|c|c|c|c|c|c|c|c|c|c|}
\hline $\begin{array}{l}\text { Year of } \\
\text { Publish }\end{array}$ & $\begin{array}{c}\text { Magnetic } \\
\text { Nanoparticle }\end{array}$ & $\begin{array}{l}\text { Heavy } \\
\text { Metal } \\
\text { Ion }\end{array}$ & $\begin{array}{l}\text { Adsorption } \\
\text { Capacity } \\
(\mathrm{mg} / \mathrm{g})^{1}\end{array}$ & $\begin{array}{l}\text { Removal } \\
\text { Efficiency } \\
(\%)\end{array}$ & $\begin{array}{l}\text { Time } \\
\text { (min) }\end{array}$ & $\mathrm{pH}$ & $\begin{array}{l}\text { Tempe- } \\
\text { rature } \\
\left({ }^{\circ} \mathrm{C}\right)\end{array}$ & $\begin{array}{c}\text { Number } \\
\text { of } \\
\text { Cycles }\end{array}$ & Ref. \\
\hline \multirow{26}{*}{2017} & $\mathrm{Fe}_{3} \mathrm{O}_{4} / \mathrm{CTAB}$ & $\mathrm{Cr}(\mathrm{VI})$ & & 95.77 & 720 & 4 & $25 \pm 0.1$ & & [290] \\
\hline & $\mathrm{Fe}_{3} \mathrm{O}_{4} @ \mathrm{Cu}_{3}(\mathrm{btc})_{2}$ & $\mathrm{~Pb}(\mathrm{II})$ & 215.05 & & & & & 4 & [291] \\
\hline & $\mathrm{Fe}_{3} \mathrm{O}_{4} @ \mathrm{Cu}_{3}(\mathrm{btc})_{2}$ & $\mathrm{Hg}(\mathrm{II})$ & 348.43 & & & & & 4 & [291] \\
\hline & $\mathrm{Fe}_{3} \mathrm{O}_{4}$-CS-L & $\mathrm{Zn}(\mathrm{II})$ & 256.41 & & 45 & 6 & 25 & 5 & [292] \\
\hline & $\mathrm{Fe}_{3} \mathrm{O}_{4}-\mathrm{CS}-\mathrm{L}$ & $\mathrm{Cd}(\mathrm{II})$ & 156.99 & & 45 & 6 & 25 & 5 & [292] \\
\hline & $\mathrm{Fe}_{3} \mathrm{O}_{4}-\mathrm{CS}-\mathrm{L}$ & $\mathrm{Pb}(\mathrm{II})$ & 128.63 & & 45 & 6 & 25 & 5 & [292] \\
\hline & $\mathrm{Fe}_{3} \mathrm{O}_{4}-\mathrm{mSiO}_{2}$ & $\mathrm{Cu}(\mathrm{II})$ & 84.4 & & & & & & [293] \\
\hline & $\mathrm{Fe}_{3} \mathrm{O}_{4}-\mathrm{mSiO}_{2}$ & $\mathrm{Cd}(\mathrm{II})$ & 80.5 & & & & & & [293] \\
\hline & $\mathrm{Fe}_{3} \mathrm{O}_{4}-\mathrm{mSiO}_{2}$ & $\mathrm{Zn}$ (II) & 72.6 & & & & & & [293] \\
\hline & $\gamma-\mathrm{Fe}_{2} \mathrm{O}_{3} @ \mathrm{CS}$ & $\mathrm{Cd}(\mathrm{II})$ & 15.2 & & 60 & 5 & $20 \pm 0.1$ & 5 & [294] \\
\hline & $\mathrm{L}-\mathrm{Cyst}-\mathrm{Fe}_{3} \mathrm{O}_{4}$ & $\mathrm{~Pb}(\mathrm{II})$ & 18.8 & & & 2 & 45 & 5 & [295] \\
\hline & $\mathrm{L}-\mathrm{Cyst}-\mathrm{Fe}_{3} \mathrm{O}_{4}$ & $\mathrm{Cr}(\mathrm{VI})$ & 34.5 & & & 6 & 45 & 5 & [295] \\
\hline & $\mathrm{MDA}-\mathrm{Fe}_{3} \mathrm{O}_{4}$ & $\mathrm{~Pb}(\mathrm{II})$ & 333.3 & & & 5 & 30 & & [296] \\
\hline & $\mathrm{ZrO}_{2}-\mathrm{Fe}_{3} \mathrm{O}_{4}$ & As(III) & 113.48 & & & 7 & & 5 & [297] \\
\hline & $\begin{array}{c}\mathrm{Fe}_{3} \mathrm{O}_{4} @ \mathrm{SiO}_{2} @ \mathrm{CS}- \\
\text { TETA-GO }\end{array}$ & $\mathrm{Cu}(\mathrm{II})$ & 324.7 & & 16 & 6 & 30 & 6 & [298] \\
\hline & $\mathrm{CoFe}_{2} \mathrm{O}_{4} @ \mathrm{SiO}_{2}-\mathrm{SH}$ & $\mathrm{Hg}(\mathrm{II})$ & 641.0 & & & 8 & 25 & 5 & [299] \\
\hline & $\mathrm{CoFe}_{2} \mathrm{O}_{4} @ \mathrm{SiO}_{2}-\mathrm{SH}$ & $\mathrm{Hg}(\mathrm{II})$ & 628.9 & & & 8 & 35 & & [299] \\
\hline & $\mathrm{CoFe}_{2} \mathrm{O}_{4} @ \mathrm{SiO}_{2}-\mathrm{SH}$ & $\mathrm{Hg}(\mathrm{II})$ & 591.7 & & & 8 & 45 & & [299] \\
\hline & $\begin{array}{c}\text { EDTA- } \\
\text { functionalized } \\
\mathrm{CoFe}_{2} \mathrm{O}_{4} \\
\text { (EDTA-MNP) }\end{array}$ & $\mathrm{Cu}(\mathrm{II})$ & 73.26 & & & 6 & 50 & & [300] \\
\hline & IMSA & $\mathrm{Pb}(\mathrm{II})$ & 133.73 & & & & & & [301] \\
\hline & IMSA & $\mathrm{As}(\mathrm{V})$ & 21.61 & & & & & & [301] \\
\hline & $\mathrm{CoFe}_{2} \mathrm{O}_{4} @ \mathrm{SiO}_{2}$ & $\mathrm{Cd}(\mathrm{II})$ & 199.9 & & & 7 & & 5 & [302] \\
\hline & $\mathrm{CoFe}_{2} \mathrm{O}_{4} @ \mathrm{SiO}_{2}$ & $\mathrm{Cu}(\mathrm{II})$ & 177.8 & & & 7 & & 5 & [302] \\
\hline & $\mathrm{CoFe}_{2} \mathrm{O}_{4} @ \mathrm{SiO}_{2}$ & $\mathrm{~Pb}(\mathrm{II})$ & 181.6 & & 30 & 7 & & 5 & [302] \\
\hline & $\begin{array}{c}\text { TEPA } \\
\text { chitosan } / \mathrm{CoFe}_{2} \mathrm{O}_{4}\end{array}$ & $\mathrm{Cu}(\mathrm{II})$ & 168.067 & & 50 & 5 & 30 & & [105] \\
\hline & $\begin{array}{c}\text { TEPA } \\
\text { chitosan } / \mathrm{CoFe}_{2} \mathrm{O}_{4}\end{array}$ & $\mathrm{~Pb}(\mathrm{II})$ & 228.311 & & 50 & 5 & 30 & & [105] \\
\hline \multirow[t]{2}{*}{2018} & $\begin{array}{c}\text { Lantana camara } \\
\text { capped iron } \\
\text { nanoparticles }\end{array}$ & $\mathrm{Ni}(\mathrm{II})$ & 227.2 & & & 6 & 60 & & [303] \\
\hline & $\mathrm{NA}-\mathrm{FeO}_{\mathrm{x}}$ & $\mathrm{Cd}(\mathrm{II})$ & 11.3 & & 60 & & & & [304] \\
\hline
\end{tabular}


Table 5. Cont.

\begin{tabular}{|c|c|c|c|c|c|c|c|c|c|}
\hline $\begin{array}{l}\text { Year of } \\
\text { Publish }\end{array}$ & $\begin{array}{c}\text { Magnetic } \\
\text { Nanoparticle }\end{array}$ & $\begin{array}{c}\text { Heavy } \\
\text { Metal } \\
\text { Ion }\end{array}$ & $\begin{array}{c}\text { Adsorption } \\
\text { Capacity } \\
\text { (mg/g) } 1\end{array}$ & $\begin{array}{l}\text { Removal } \\
\text { Efficiency } \\
(\%)\end{array}$ & $\begin{array}{l}\text { Time } \\
\text { (min) }\end{array}$ & $\mathrm{pH}$ & $\begin{array}{l}\text { Tempe- } \\
\text { rature } \\
\left({ }^{\circ} \mathrm{C}\right)\end{array}$ & $\begin{array}{c}\text { Number } \\
\text { of } \\
\text { Cycles }\end{array}$ & Ref. \\
\hline \multirow{24}{*}{2018} & $\mathrm{NA}-\mathrm{FeO}_{x}$ & $\mathrm{Cu}(\mathrm{II})$ & 12.3 & & 60 & & & & [304] \\
\hline & $\begin{array}{c}\text { Graphene } \\
\text { oxide- } \mathrm{Fe}_{3} \mathrm{O}_{4}\end{array}$ & $\mathrm{~Pb}(\mathrm{II})$ & 373.14 & & 10 & 6 & & & [305] \\
\hline & PAE-AAm-g-MNPs & $\mathrm{Cu}(\mathrm{II})$ & 30.34 & & & 6 & & 7 & [306] \\
\hline & $\mathrm{Fe}_{3} \mathrm{O}_{4} / \mathrm{LDH}-\mathrm{AM}$ & $\mathrm{Cu}(\mathrm{II})$ & 64.66 & & 240 & & & 5 & [307] \\
\hline & $\mathrm{Fe}_{3} \mathrm{O}_{4} / \mathrm{LDH}-\mathrm{AM}$ & $\mathrm{Cd}(\mathrm{II})$ & 74.06 & & 240 & & & 5 & [307] \\
\hline & $\mathrm{Fe}_{3} \mathrm{O}_{4} / \mathrm{LDH}-\mathrm{AM}$ & $\mathrm{Pb}(\mathrm{II})$ & 266.6 & & 180 & & & 5 & [307] \\
\hline & TEPA-GO/ $\mathrm{MnFe}_{2} \mathrm{O}_{4}$ & $\mathrm{~Pb}(\mathrm{II})$ & 263.2 & & & 5.5 & 30 & 4 & [308] \\
\hline & $\mathrm{GO} / \mathrm{MnFe}_{2} \mathrm{O}_{4}$ & $\mathrm{~Pb}(\mathrm{II})$ & 133.3 & & & & & & [308] \\
\hline & $\mathrm{MnFe}_{2} \mathrm{O}_{4}-\mathrm{BC}$ & $\mathrm{Cd}(\mathrm{II})$ & 181.49 & & & 7 & 25 & 5 & [309] \\
\hline & $\mathrm{CoFe}_{2} \mathrm{O}_{4} @ \mathrm{SiO}_{2}$ & $\mathrm{Hg}(\mathrm{II})$ & 149.3 & & & 7 & 25 & 5 & [310] \\
\hline & $\mathrm{CoFe}_{2} \mathrm{O}_{4} @ \mathrm{SiO}_{2}$ & $\mathrm{Hg}(\mathrm{II})$ & 144.9 & & & 7 & 35 & & [310] \\
\hline & $\mathrm{CoFe}_{2} \mathrm{O}_{4} @ \mathrm{SiO}_{2}$ & $\mathrm{Hg}(\mathrm{II})$ & 131.6 & & & 7 & 45 & & [310] \\
\hline & p-BNMR@ $\mathrm{Fe}_{3} \mathrm{O}_{4}$ & $\mathrm{~Pb}(\mathrm{II})$ & 249.5 & & & 5.5 & 25 & & [311] \\
\hline & Aminated- $\mathrm{Fe}_{3} \mathrm{O}_{4}$ & $\mathrm{Cr}(\mathrm{VI})$ & 19.5 & & & 3.5 & & 3 & [312] \\
\hline & Aminated- $\mathrm{Fe}_{3} \mathrm{O}_{4}$ & $\mathrm{~Pb}(\mathrm{II})$ & 21.2 & & & 3.5 & & 3 & [312] \\
\hline & $\mathrm{Fe}_{3} \mathrm{O}_{4} / \operatorname{poly}\left(\mathrm{C}_{3} \mathrm{~N}_{3} \mathrm{~S}_{3}\right)$ & $\mathrm{Pb}(\mathrm{II})$ & 232.6 & & & 6 & 25 & 7 & [313] \\
\hline & $\mathrm{Fe}_{3} \mathrm{O}_{4} / \operatorname{poly}\left(\mathrm{C}_{3} \mathrm{~N}_{3} \mathrm{~S}_{3}\right)$ & $\mathrm{Hg}(\mathrm{II})$ & 344.8 & & & 6 & 25 & 7 & [313] \\
\hline & $\mathrm{rGO}-\mathrm{PDTC} / \mathrm{Fe}_{3} \mathrm{O}_{4}$ & $\mathrm{Cu}(\mathrm{II})$ & 113.64 & & & 5 & 25 & 5 & [314] \\
\hline & $\mathrm{rGO}-\mathrm{PDTC} / \mathrm{Fe}_{3} \mathrm{O}_{4}$ & $\mathrm{Cd}(\mathrm{II})$ & 116.28 & & & 6 & 25 & 5 & [314] \\
\hline & rGO-PDTC $/ \mathrm{Fe}_{3} \mathrm{O}_{4}$ & $\mathrm{~Pb}(\mathrm{II})$ & 147.06 & & & 6 & 25 & 5 & [314] \\
\hline & $\mathrm{rGO}-\mathrm{PDTC} / \mathrm{Fe}_{3} \mathrm{O}_{4}$ & $\mathrm{Hg}(\mathrm{II})$ & 181.82 & & & 6 & 25 & 5 & [314] \\
\hline & $\mathrm{d}-\mathrm{MoS} 2 / \mathrm{Fe}_{3} \mathrm{O}_{4}$ & $\mathrm{Hg}(\mathrm{II})$ & 425.5 & & 20 & & & 5 & [315] \\
\hline & $\begin{array}{c}\mathrm{CHT} / \mathrm{ALG} / \mathrm{Fe}_{3} \mathrm{O}_{4} \\
@ \mathrm{SiO}_{2} \\
\text { (8 beads) }\end{array}$ & $\mathrm{Pb}(\mathrm{II})$ & 243.77 & & & 4.2 & 20 & & [316] \\
\hline & $\begin{array}{c}\mathrm{CHT} / \mathrm{ALG} / \mathrm{Fe}_{3} \mathrm{O}_{4} \\
@ \mathrm{SiO}_{2} \\
(1 \text { bead })\end{array}$ & $\mathrm{Pb}(\mathrm{II})$ & 228.73 & & & 4.2 & 20 & & [316] \\
\hline \multirow{6}{*}{2019} & $\mathrm{Fe}_{3} \mathrm{O}_{4} / \mathrm{BC} / \mathrm{AC}$ & $\mathrm{Pb}(\mathrm{II})$ & 169.78 & & & 5 & 25 & 5 & [317] \\
\hline & $\mathrm{Fe}_{3} \mathrm{O}_{4} @ \mathrm{FePO}_{4}$ & $\mathrm{Cd}(\mathrm{II})$ & 13.51 & & 15 & 7 & & & [318] \\
\hline & $\begin{array}{c}\mathrm{Fe}_{3} \mathrm{O}_{4} @ \mathrm{SiO}_{2}-\mathrm{NH}- \\
\mathrm{MFL}\end{array}$ & $\mathrm{Pb}(\mathrm{II})$ & 150.33 & & 0.5 & 5 & & & [156] \\
\hline & $\begin{array}{c}\mathrm{Fe}_{3} \mathrm{O}_{4} @ \mathrm{SiO}_{2}-\mathrm{NH}- \\
\mathrm{MFL}\end{array}$ & $\mathrm{Cu}(\mathrm{II})$ & 70.7 & & 0.5 & 5 & & & [156] \\
\hline & DTT-Fe $\mathrm{O}_{4} @ \mathrm{Au}$ & As(III) & & 68.8 & & 5 & & & [319] \\
\hline & $\begin{array}{c}\mathrm{rGO}- \\
\operatorname{poly}\left(\mathrm{C}_{3} \mathrm{~N}_{3} \mathrm{~S}_{3}\right) / \mathrm{Fe}_{3} \mathrm{O}_{4}\end{array}$ & $\mathrm{~Pb}(\mathrm{II})$ & 270.3 & & 60 & 6 & 25 & 15 & [320] \\
\hline
\end{tabular}


Table 5. Cont.

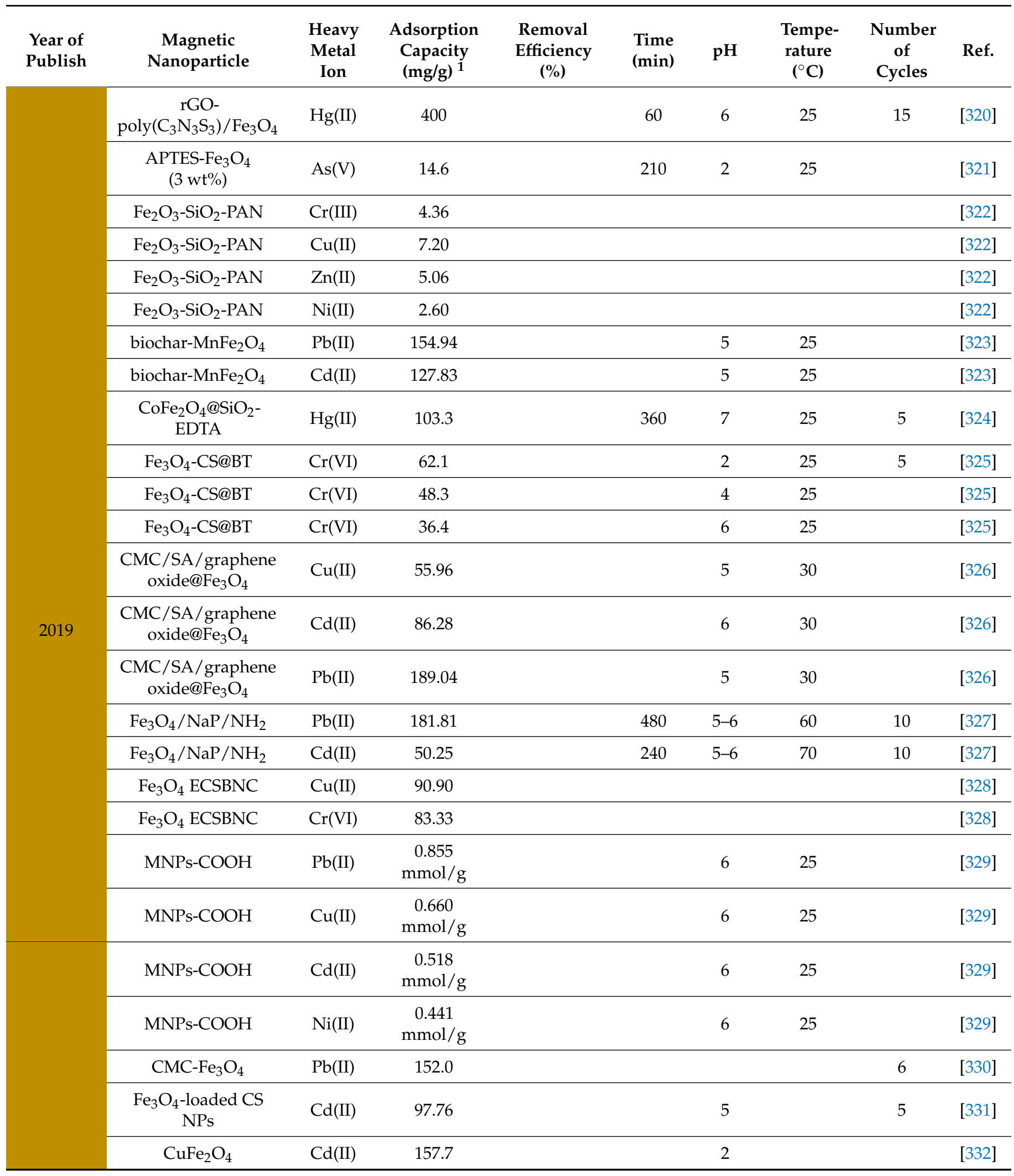


Table 5. Cont.

\begin{tabular}{|c|c|c|c|c|c|c|c|c|c|}
\hline $\begin{array}{l}\text { Year of } \\
\text { Publish }\end{array}$ & $\begin{array}{c}\text { Magnetic } \\
\text { Nanoparticle }\end{array}$ & $\begin{array}{c}\text { Heavy } \\
\text { Metal } \\
\text { Ion }\end{array}$ & $\begin{array}{l}\text { Adsorption } \\
\text { Capacity } \\
(\mathrm{mg} / \mathrm{g})^{1}\end{array}$ & $\begin{array}{c}\text { Removal } \\
\text { Efficiency } \\
(\%)\end{array}$ & $\begin{array}{l}\text { Time } \\
\text { (min) }\end{array}$ & $\mathrm{pH}$ & $\begin{array}{l}\text { Tempe- } \\
\text { rature } \\
\left({ }^{\circ} \mathrm{C}\right)\end{array}$ & $\begin{array}{c}\text { Number } \\
\text { of } \\
\text { Cycles }\end{array}$ & Ref. \\
\hline \multirow{4}{*}{2019} & $\mathrm{CoFe}_{2} \mathrm{O}_{4}$ & $\mathrm{~Pb}(\mathrm{II})$ & 63.1 & & & 12 & & & [332] \\
\hline & $\begin{array}{l}\mathrm{HP}-\beta-\mathrm{CD}- \\
\mathrm{GO} / \mathrm{Fe}_{3} \mathrm{O}_{4}\end{array}$ & $\mathrm{Cu}(\mathrm{II})$ & 17.91 & & & 6 & & 5 & [333] \\
\hline & $\begin{array}{l}\mathrm{HP}-\beta-\mathrm{CD}- \\
\mathrm{GO} / \mathrm{Fe}_{3} \mathrm{O}_{4}\end{array}$ & $\mathrm{~Pb}(\mathrm{II})$ & 50.39 & & & 5 & & 5 & [333] \\
\hline & $\begin{array}{c}\text { Bentonite } / \mathrm{CoFe}_{2} \mathrm{O}_{4} \\
@ \mathrm{MnO}_{2}-\mathrm{NH}_{2}\end{array}$ & $\mathrm{Cd}(\mathrm{II})$ & 115.79 & 98.88 & & & & & [334] \\
\hline \multirow{24}{*}{2020} & $\begin{array}{l}\mathrm{Fe}_{3} \mathrm{O}_{4} @ \mathrm{SiO}_{2} \\
@ \mathrm{GLYMO}(\mathrm{S})\end{array}$ & $\mathrm{Cd}(\mathrm{II})$ & 80.64 & & 55 & 7 & & 5 & [335] \\
\hline & $\begin{array}{l}\mathrm{Fe}_{3} \mathrm{O}_{4} @ \mathrm{SiO}_{2} \\
@ \mathrm{GLYMO}(\mathrm{S})\end{array}$ & $\mathrm{Pb}(\mathrm{II})$ & 93.5 & & 55 & 7 & & 5 & [335] \\
\hline & MGO & $\mathrm{Pb}(\mathrm{II})$ & 200 & & 30 & 5 & & & [167] \\
\hline & MGO & $\mathrm{Cr}(\mathrm{III})$ & 24.33 & & 30 & 6 & & & [167] \\
\hline & MGO & $\mathrm{Cu}(\mathrm{II})$ & 62.89 & & 30 & 6 & & & [167] \\
\hline & MGO & $\mathrm{Zn}(\mathrm{II})$ & 63.69 & & 30 & 7 & & & [167] \\
\hline & MGO & $\mathrm{Ni}(\mathrm{II})$ & 51.02 & & 30 & 8 & & & [167] \\
\hline & $\begin{array}{l}\text { Proanthocyanidin- } \\
\text { functionalized } \mathrm{Fe}_{3} \mathrm{O}_{4}\end{array}$ & $\mathrm{Cu}(\mathrm{II})$ & 18.8 & & 30 & 8 & & 5 & [336] \\
\hline & $\begin{array}{l}\text { Proanthocyanidin- } \\
\text { functionalized } \mathrm{Fe}_{3} \mathrm{O}_{4}\end{array}$ & $\mathrm{Cd}(\mathrm{II})$ & 20.9 & & 30 & 8 & & & [336] \\
\hline & $\begin{array}{l}\text { Proanthocyanidin- } \\
\text { functionalized } \\
\mathrm{Fe}_{3} \mathrm{O}_{4}\end{array}$ & $\mathrm{~Pb}(\mathrm{II})$ & 21.5 & & 30 & 8 & & & [336] \\
\hline & Ggh-g-PAcM $/ \mathrm{Fe}_{3} \mathrm{O}_{4}$ & $\mathrm{Cu}(\mathrm{II})$ & 224.8 & & & & 30 & & [337] \\
\hline & Ggh-g-PAcM/ $\mathrm{Fe}_{3} \mathrm{O}_{4}$ & $\mathrm{Hg}(\mathrm{II})$ & 213.8 & & & & 30 & & [337] \\
\hline & $\begin{array}{c}\mathrm{Fe}_{3} \mathrm{O}_{4}-\mathrm{GO} \text { hybrid } \\
(9: 1)\end{array}$ & $\mathrm{Pb}(\mathrm{II})$ & 107.56 & & & 6 & & & [338] \\
\hline & $\begin{array}{c}\mathrm{Fe}_{3} \mathrm{O}_{4} \text {-GO hybrid } \\
(5: 1)\end{array}$ & $\mathrm{Pb}(\mathrm{II})$ & 151.22 & & & 6 & & & [338] \\
\hline & $\mathrm{M}-45 \mathrm{OA}$ & $\mathrm{Pb}(\mathrm{II})$ & 42.553 & & & 6 & & & [338] \\
\hline & $\mathrm{M}-45 \mathrm{OA}$ & $\mathrm{Zn}(\mathrm{II})$ & 42.919 & & & 6 & & & [339] \\
\hline & $\mathrm{M}-45 \mathrm{OA}$ & $\mathrm{Cd}(\mathrm{II})$ & 42.373 & & & 7 & & & [339] \\
\hline & M-55 OA & $\mathrm{Pb}(\mathrm{II})$ & 41.841 & & & 6 & & & [339] \\
\hline & M-55 OA & $\mathrm{Zn}(\mathrm{II})$ & 42.735 & & & 6 & & & [339] \\
\hline & M-55 OA & $\mathrm{Cd}(\mathrm{II})$ & 42.017 & & & 7 & & & [339] \\
\hline & M-55+ & $\mathrm{Pb}(\mathrm{II})$ & 40.816 & & & 6 & & & [339] \\
\hline & M-55+ & $\mathrm{Zn}(\mathrm{II})$ & 40.816 & & & 6 & & & [339] \\
\hline & M-55+ & $\mathrm{Cd}(\mathrm{II})$ & 39.216 & & & 7 & & & [339] \\
\hline & $\mathrm{BNNF} @ \mathrm{Fe}_{3} \mathrm{O}_{4}$ & $\mathrm{~Pb}(\mathrm{II})$ & 203.75 & & 50 & & 25 & & [340] \\
\hline
\end{tabular}


Table 5. Cont.

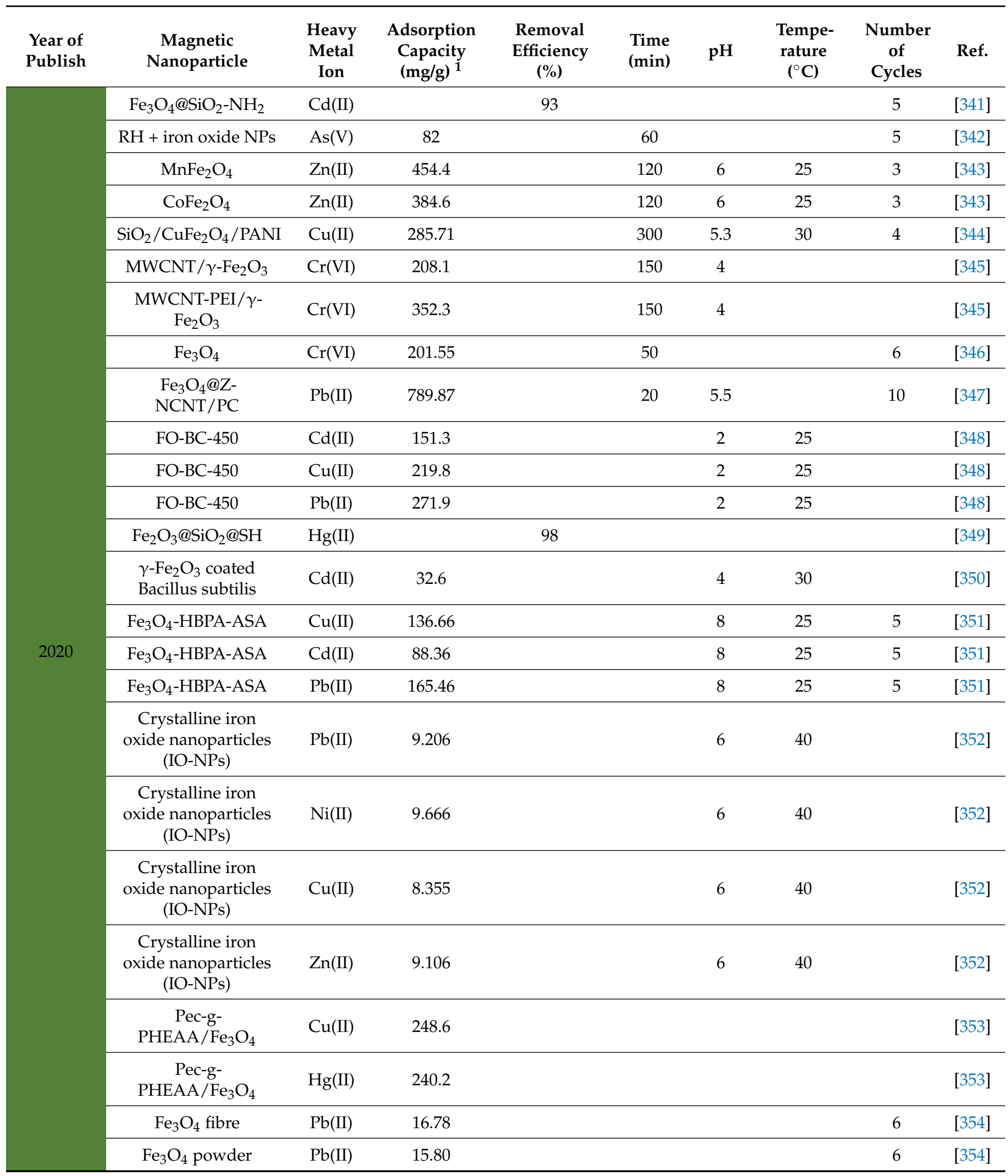


Table 5. Cont.

\begin{tabular}{|c|c|c|c|c|c|c|c|c|c|}
\hline $\begin{array}{l}\text { Year of } \\
\text { Publish }\end{array}$ & $\begin{array}{c}\text { Magnetic } \\
\text { Nanoparticle }\end{array}$ & $\begin{array}{c}\text { Heavy } \\
\text { Metal } \\
\text { Ion }\end{array}$ & $\begin{array}{l}\text { Adsorption } \\
\text { Capacity } \\
(\mathrm{mg} / \mathrm{g})^{1}\end{array}$ & $\begin{array}{c}\text { Removal } \\
\text { Efficiency } \\
(\%)\end{array}$ & $\begin{array}{l}\text { Time } \\
(\mathrm{min})\end{array}$ & $\mathrm{pH}$ & $\begin{array}{l}\text { Tempe- } \\
\text { rature } \\
\left({ }^{\circ} \mathrm{C}\right)\end{array}$ & $\begin{array}{l}\text { Number } \\
\text { of } \\
\text { Cycles }\end{array}$ & Ref. \\
\hline \multirow{11}{*}{2020} & $\begin{array}{l}\text { Iron oxide (MNPs) } \\
\text { grafted (HPG) }\end{array}$ & $\mathrm{Cu}(\mathrm{II})$ & $\begin{array}{c}0.700 \\
\mathrm{mg} / \mathrm{mg}\end{array}$ & & 120 & 9 & 20 & & [355] \\
\hline & $\begin{array}{l}\text { Iron oxide (MNPs) } \\
\text { grafted (HPG) }\end{array}$ & $\mathrm{Ni(II)}$ & $\begin{array}{c}0.451 \\
\mathrm{mg} / \mathrm{mg}\end{array}$ & & 120 & 9 & 20 & & [355] \\
\hline & Nano-CI & $\mathrm{Pb}(\mathrm{II})$ & $\begin{array}{c}1900 \\
\mu \mathrm{mol} / \mathrm{g}\end{array}$ & & 30 & 6 & & & [356] \\
\hline & Nano-CI & $\mathrm{Cu}(\mathrm{II})$ & $\begin{array}{c}2250 \\
\mu \mathrm{mol} / \mathrm{g}\end{array}$ & & 30 & 7 & & & [356] \\
\hline & Nano-CI & $\mathrm{Cd}(\mathrm{II})$ & $850 \mu \mathrm{mol} / \mathrm{g}$ & & 30 & 7 & & & [356] \\
\hline & Nano-CIC & $\mathrm{Pb}(\mathrm{II})$ & $\begin{array}{c}2700 \\
\mu \mathrm{mol} / \mathrm{g}\end{array}$ & & 30 & 4 & & & [356] \\
\hline & Nano-CIC & $\mathrm{Cu}(\mathrm{II})$ & $\begin{array}{c}4250 \\
\mu \mathrm{mol} / \mathrm{g}\end{array}$ & & 30 & 6 & & & [356] \\
\hline & Nano-CIC & $\mathrm{Cd}(\mathrm{II})$ & $\begin{array}{c}1800 \\
\mu \mathrm{mol} / \mathrm{g}\end{array}$ & & 30 & 6 & & & [356] \\
\hline & Nano-CIS & $\mathrm{Pb}(\mathrm{II})$ & $\begin{array}{c}2600 \\
\mu \mathrm{mol} / \mathrm{g}\end{array}$ & & 10 & 4 & & & [356] \\
\hline & Nano-CIS & $\mathrm{Cu}(\mathrm{II})$ & $\begin{array}{c}4700 \\
\mu \mathrm{mol} / \mathrm{g}\end{array}$ & & 10 & 6 & & & [356] \\
\hline & Nano-CIS & $\mathrm{Cd}(\mathrm{II})$ & $\begin{array}{c}1900 \\
\mu \mathrm{mol} / \mathrm{g}\end{array}$ & & 10 & 6 & & & [356] \\
\hline
\end{tabular}

${ }^{1}$ Cases with unit of adsorption capacity that is different from $\mathrm{mg} / \mathrm{g}$ are listed next to the respective value.

A crucial stage in the evaluation of the applicability of the experimental methods is the possibility of reducing the production cost and time. This reduction could be achieved by reusing the existing nanoparticles; thus the stages of synthesis and characterization could be avoided. Desorption processes may occur either by thermal treatment or through suitable desorbing agents and are necessary for recycling [171], so the nanoparticles could be used again. Among the selected articles, all have employed desorbing agents during the desorption process. The performance of the desorption process for magnetic nanoparticles is directly related to the size, coating, magnetic behaviour [159] and $\mathrm{pH}$ of the solution [357], while other critical factors are the kind of regenerative solutions (i.e., $\mathrm{NaOH}, \mathrm{HCl}$ ) but also their concentration. For example, $2 \mathrm{M}$ of $\mathrm{HNO}_{3}$ has a desorption efficiency of $\mathrm{Cr}$ (VI) equal to $73 \%$, but the efficiency drops to $20 \%$ when $0.1 \mathrm{M}$ of $\mathrm{HNO}_{3}$ is employed [231]. Additional benefits of the desorption process are the limited cost of desorbing agents and the time of the process, which could be achieved in less than $1 \mathrm{~h}$. The usage of the desorption process is enhanced by the ease of collection, which comes from the selectivity of the paramagnetic nanoparticles assisting the technique, because they could be readily separated from the solution when a magnetic field is applied; thus iron oxide nanoparticles are more preferable to other nanoparticles with no magnetic cores. Additionally, computational fluid dynamics could be employed at this stage, reducing the cost of the materials. The major advantage of computational water treatment methods compared with an experimental method is that the steps of synthesis and characterization of magnetic nanoparticles are not time-consuming since they do not exist. The aims of microfluidic mixing and driving simulations for water purification from heavy metal ions are to achieve rapid mixing and desired guidance of nanoparticles $[48,51,59,358]$. 
Nonetheless, the effectiveness of the adsorption-desorption process is evaluated by the efficiency of heavy metal adsorption after each recycling. An ideal adsorbent is considered to be one that simultaneously possesses high adsorption capability and high desorption efficiency [359]. Of critical importance is the effectiveness of the process in several studies, in which up to five cycles of adsorption without a significant decrease in efficiency have been achieved [360]. In addition, high adsorption and desorption efficiencies equal to $98.4 \%$ after seven cycles [306] and 98\% (constant) for over 15 cycles have been reported [171]. The deterioration of active binding sites on the surface absorbent during recycling results in a decrease in efficiency. It should be noted, however, that in some cases after the desorption process, the adsorption efficiency did not decrease, but instead, it increased in the next cycle [361]; this phenomenon is based on the increase in the positively charged surface of nanoparticles, which leads to increased electrostatic attraction forces between the iron oxide nanoparticles and the pollutant. Recycling of the adsorbent is important to obtain the process that enhances the viability of the adsorption method.

The recycling efficiency and adsorption capacity for each cycle are presented in Tables 6 and 7, respectively. We must mention here that there are very few articles that have investigated the adsorption capacity. This fact has a negative impact on the applicability of the method. During the literature review were recorded cases where the recycling effectiveness was measured with adsorption capacity instead of efficiency. Additionally, both adsorption and desorption efficiency decreased through the regeneration cycles and due to the difficulty in reversing adsorption [362].

Table 6. Recycling efficiency (\%).

\begin{tabular}{|c|c|c|c|c|c|c|}
\hline Pollutant & 1st Cycle & 2nd Cycle & 3rd Cycle & 4th Cycle & 5th Cycle & Ref. \\
\hline $\mathrm{Pb}(\mathrm{II})$ & 90.12 & 88.05 & 85.65 & 81.35 & & [360] \\
\hline $\mathrm{Cu}(\mathrm{II})$ & 93.70 & & & & 58.66 & [196] \\
\hline $\mathrm{Pb}(\mathrm{II})$ & & & & & 90 & [197] \\
\hline $\mathrm{Cr}(\mathrm{VI})$ & 55 & 88 & & 90 & & [361] \\
\hline $\mathrm{Pb}(\mathrm{II})$ & 97.34 & & & & 90 & [175] \\
\hline $\mathrm{Hg}(\mathrm{II})$ & & & & & $\geq 96$ & [157] \\
\hline $\mathrm{Pb}$ (II) & & & & & 90 & [201] \\
\hline $\mathrm{Pb}(\mathrm{II})$ & 93.5 & & & & 89.3 & [335] \\
\hline $\mathrm{Cu}(\mathrm{II})$ & 80.64 & & & & 73.3 & [335] \\
\hline $\operatorname{As}(\mathrm{V})$ & 95 & & & & 56 & [342] \\
\hline $\mathrm{Cd}(\mathrm{II})$ & & & & 76.4 & & [348] \\
\hline $\mathrm{Cu}(\mathrm{II})$ & & & & 80.4 & & [348] \\
\hline $\mathrm{Pb}(\mathrm{II})$ & & & & 70.2 & & [348] \\
\hline $\mathrm{Hg}(\mathrm{II})$ & $>90$ & & & & $\sim 75.5$ & [324] \\
\hline $\mathrm{Cd}(\mathrm{II})$ & 98.8 & 95.1 & 91.7 & 84.6 & 78.3 & [294] \\
\hline $\mathrm{Cd}(\mathrm{II})$ & 99.96 & & & 97.25 & & [302] \\
\hline $\mathrm{Cu}(\mathrm{II})$ & 88.05 & & & 84.15 & & [302] \\
\hline $\mathrm{Pb}(\mathrm{II})$ & 90.79 & & & 87.12 & & [302] \\
\hline $\mathrm{Pb}(\mathrm{II})$ & 96.2 & & & & 86.4 & [257] \\
\hline $\mathrm{Hg}(\mathrm{II})$ & 95.1 & & & & 85.9 & [257] \\
\hline $\mathrm{Cu}(\mathrm{II})$ & 96.5 & & & & 87.6 & [257] \\
\hline
\end{tabular}


Table 7. Recycling adsorption capacity (mg/g).

\begin{tabular}{cccccccc}
\hline \multirow{2}{*}{ Pollutant } & \multirow{2}{*}{ 1st Cycle } & $\begin{array}{c}\text { 2nd } \\
\text { Cycle }\end{array}$ & $\begin{array}{c}\text { 3rd } \\
\text { Cycle }\end{array}$ & $\begin{array}{c}\text { 4th } \\
\text { Cycle }\end{array}$ & $\begin{array}{c}\text { 5th } \\
\text { Cycle }\end{array}$ & $\begin{array}{c}\text { 6th } \\
\text { Cycle }\end{array}$ & Ref. \\
\hline $\mathrm{Cr}(\mathrm{VI})$ & 132.56 & 130.62 & 127.52 & 125.97 & 124.42 & 121.71 & {$[346]$} \\
\hline $\mathrm{Cu}(\mathrm{II})$ & 197.5 & 196.5 & 195 & 194.4 & & & {$[344]$} \\
\hline
\end{tabular}

\section{Conclusions}

During the last decade, nanotechnology has led to great progress in several fields, including water purification and heavy metal removal, with a large number of published articles. Therefore, a need arises for a frame of water purification from heavy metals using the adsorption method, which includes not only the main findings during the last decade but also all the phases of the technique. The success of the method is based not only on adsorption efficiency or capacity but also on applicability.

We must consider that a specific bare or modified nanoparticle that can act as a panacea for water purification from all heavy metals does not exist. This is proved by the experimental results, which show that adsorption capacity differs between pollutants, while all the other parameters remain constant. However, according to the literature review, researchers seem to have investigated more capping agents, such as $\mathrm{SiO}_{2}$, amino groups, and graphene oxide. Additionally, the removal efficiency is different for various water sources (lake, river, groundwater, tap water, and sea) under the same heavy metal ion and adsorbent [187]. Moreover, from the context that is delimited above, it is clear that for each parameter exists a critical point where the adsorption efficiency is reaching a maximum, resulting in difficulty in scaling up. It is clear from the equilibrium time that the scale-up could be more realistic in reservoirs rather than in the case of continuous flow inside pipes. The ideal range of $\mathrm{pH}$ for the maximum adsorption without dependence on the pollutant is between 5 and 7 . However, the main findings that are presented in this review almost reach the optimum efficiency. An encouraging fact is that the majority of experiments take place at room temperature with high adsorption capacity. The ability to reuse nanoparticles after desorption constitutes a significant parameter of applicability. Therefore, the preparation of each adsorbent must be targeted for the removal of a specific pollutant by the adsorption method.

Author Contributions: Conceptualization, C.L. and E.K.; methodology, A.P.; formal analysis, C.L.; investigation, C.L., A.P. and E.K.; writing-original draft preparation, C.L.; writing-review and editing, T.E.K. and I.E.S.; supervision, I.E.S.; funding acquisition, T.E.K. All authors have read and agreed to the published version of the manuscript.

Funding: This research is co-financed by Greece and the European Union (European Social FundESF) through the Operational Programme «Human Resources Development, Education and Lifelong Learning 2014-2020» in the context of the project "Magnetic driving of nanoparticles in human artery networks" (MIS: 5048923).

Institutional Review Board Statement: Not applicable.

Informed Consent Statement: Not applicable.

Data Availability Statement: Data is contained within the article and references therein.

Conflicts of Interest: The authors declare no conflict of interest.

\section{References}

1. Olvera, R.C.; Silva, S.L.; Robles-Belmont, E.; Lau, E.Z. Review of Nanotechnology Value Chain for Water Treatment Applications in Mexico. Resour. Technol. 2017, 3, 1-11. [CrossRef]

2. Jiang, L.; Jiapaer, G.; Bao, A.; Yuan, Y.; Zheng, G.; Guo, H.; Yu, T.; De Maeyer, P. The Effects of Water Stress on Croplands in the Aral Sea Basin. J. Clean. Prod. 2020, 254, 120114. [CrossRef]

3. Lee, U.; Xu, H.; Daystar, J.; Elgowainy, A.; Wang, M. AWARE-US: Quantifying Water Stress Impacts of Energy Systems in the United States. Sci. Total Environ. 2019, 648, 1313-1322. [CrossRef] [PubMed] 
4. Ward, F.A.; Mayer, A.S.; Garnica, L.A.; Townsend, N.T.; Gutzler, D.S. The Economics of Aquifer Protection Plans under Climate Water Stress: New Insights from Hydroeconomic Modeling. J. Hydrol. 2019, 576, 667-684. [CrossRef]

5. Dinar, A.; Tieu, A.; Huynh, H. Water Scarcity Impacts on Global Food Production. Glob. Food Sec. 2019, 23, 212-226. [CrossRef]

6. Zhao, H.; Qu, S.; Guo, S.; Zhao, H.; Liang, S.; Xu, M. Virtual Water Scarcity Risk to Global Trade under Climate Change. J. Clean. Prod. 2019, 230, 1013-1026. [CrossRef]

7. Jia, X.; Klemeš, J.J.; Alwi, S.R.W.; Varbanov, P.S. Regional Water Resources Assessment Using Water Scarcity Pinch Analysis. Resour. Conserv. Recycl. 2020, 157, 104749. [CrossRef]

8. Moursi, H.; Kim, D.; Kaluarachchi, J.J. A Probabilistic Assessment of Agricultural Water Scarcity in a Semi-Arid and SnowmeltDominated River Basin under Climate Change. Agric. Water Manag. 2017, 193, 142-152. [CrossRef]

9. Defining Water Scarcity, Water Stress, and Water Risk. Available online: https://pacinst.org/water-definitions/ (accessed on 14 January 2019).

10. McNabb, D. The Population Growth Barrier. In Global Pathways to Water Sustainability, 1st ed.; Springer International Publishing: Berlin/Heidelberg, Germany, 2019; pp. 67-81. [CrossRef]

11. McNabb, D. Alternative Sources of Water Supply. In Global Pathways to Water Sustainability, 1st ed.; Springer International Publishing: Berlin/Heidelberg, Germany, 2019; pp. 251-262. [CrossRef]

12. Omer, A.; Elagib, N.A.; Zhuguo, M.; Saleem, F.; Mohammed, A. Water Scarcity in the Yellow River Basin under Future Climate Change and Human Activities. Sci. Total Environ. 2020, 749, 141446. [CrossRef] [PubMed]

13. Sharma, S.; Sanghi, R.; Mudhoo, A. Green Practices to Save Our Precious "Water Resource." In Advances in Water Treatment and Pollution Prevention; Sharma, S., Ed.; Springer Netherlands: Dordrecht, The Netherlands, 2012; pp. 1-36. [CrossRef]

14. Liu, J.; Liu, Q.; Yang, H. Assessing Water Scarcity by Simultaneously Considering Environmental Flow Requirements, Water Quantity, and Water Quality. Ecol. Indic. 2016, 60, 434-441. [CrossRef]

15. Boholm, Å.; Prutzer, M. Experts' Understandings of Drinking Water Risk Management in a Climate Change Scenario. Clim. Risk Manag. 2017, 16, 133-144. [CrossRef]

16. Joseph, L.; Jun, B.-M.; Flora, J.R.V.; Park, C.M.; Yoon, Y. Removal of Heavy Metals from Water Sources in the Developing World Using Low-Cost Materials: A Review. Chemosphere 2019, 229, 142-159. [CrossRef]

17. Hao, X.; Chen, G.; Yuan, Z. Water in China. Water Res. 2020, 169, 115256. [CrossRef] [PubMed]

18. Mo, W.; Zhang, Q. Energy-Nutrients-Water Nexus: Integrated Resource Recovery in Municipal Wastewater Treatment Plants. J. Environ. Manag. 2013, 127, 255-267. [CrossRef]

19. Xia, J.; Duan, Q.-Y.; Luo, Y.; Xie, Z.-H.; Liu, Z.-Y.; Mo, X.-G. Climate Change and Water Resources: Case Study of Eastern Monsoon Region of China. Adv. Clim. Chang. Res. 2017, 8, 63-67. [CrossRef]

20. Peirce, J.J.; Weiner, R.F.; Vesilind, P.A.; Peirce, J.J.; Weiner, R.F.; Vesilind, P.A. Water Pollution. Environ. Pollut. Control. 1998, 31-55. [CrossRef]

21. Wasewar, K.L.; Singh, S.; Kansal, S.K. Process intensification of treatment of inorganic water pollutants. In Inorganic Pollutants in Water; Devi, P., Singh, P., Kansal, S.K., Eds.; Elsevier: Amsterdam, The Netherlands, 2020; pp. 245-271. [CrossRef]

22. Orubu, C.O.; Omotor, D.G. Environmental Quality and Economic Growth: Searching for Environmental Kuznets Curves for Air and Water Pollutants in Africa. Energy Policy 2011, 39, 4178-4188. [CrossRef]

23. Liu, H.; Feng, S.; Du, X.; Zhang, N.; Liu, Y. Comparison of Three Sorbents for Organic Pollutant Removal in Drinking Water. Energy Procedia 2011, 5, 985-990. [CrossRef]

24. Ahmed, M.J.; Hameed, B.H.; Hummadi, E.H. Review on Recent Progress in Chitosan/Chitin-Carbonaceous Material Composites for the Adsorption of Water Pollutants. Carbohydr. Polym. 2020, 247, 116690. [CrossRef]

25. Kim, K.H.; Keller, A.A.; Yang, J.K. Removal of Heavy Metals from Aqueous Solution Using a Novel Composite of Recycled Materials. Colloids Surfaces A Physicochem. Eng. Asp. 2013, 425, 6-14. [CrossRef]

26. Hu, G.; Bakhtavar, E.; Hewage, K.; Mohseni, M.; Sadiq, R. Heavy Metals Risk Assessment in Drinking Water: An Integrated Probabilistic-Fuzzy Approach. J. Environ. Manag. 2019, 250, 109514. [CrossRef]

27. Khan, M.U.; Muhammad, S.; Malik, R.N.; Khan, S.A.; Tariq, M. Heavy Metals Potential Health Risk Assessment through Consumption of Wastewater Irrigated Wild Plants: A Case Study. Hum. Ecol. Risk Assess. 2016, 22, 141-152. [CrossRef]

28. Fu, F.; Wang, Q. Removal of Heavy Metal Ions from Wastewaters: A Review. J. Environ. Manag. 2011, 92, 407-418. [CrossRef]

29. Sarkar, A.; Paul, B. The Global Menace of Arsenic and Its Conventional Remediation-A Critical Review. Chemosphere 2016, 158, 37-49. [CrossRef]

30. Barakat, M.A. New Trends in Removing Heavy Metals from Industrial Wastewater. Arab. J. Chem. 2011, 4, 361-377. [CrossRef]

31. Babel, S.; Kurniawan, T.A. Low-Cost Adsorbents for Heavy Metals Uptake from Contaminated Water: A Review. J. Hazard. Mater. 2003, 97, 219-243. [CrossRef]

32. Stohs, S.J.; Bagchi, D. Oxidative Mechanisms in the Toxicity of Metal Ions. Free. Radic. Biol. Med. 1995, 18, 321-336. [CrossRef]

33. Parmar, M.; Thakur, L.S. Heavy Metal Cu, Ni and Zn: Toxicity, Health Hazards and Their Removal. Int. J. Plant Sci. 2013, 3, 143-157.

34. Vardhan, K.H.; Kumar, P.S.; Panda, R.C. A Review on Heavy Metal Pollution, Toxicity and Remedial Measures: Current Trends and Future Perspectives. J. Mol. Liq. 2019, 290, 111197. [CrossRef]

35. Kim, J.-J.; Kim, Y.-S.; Kumar, V. Heavy Metal Toxicity: An Update of Chelating Therapeutic Strategies. J. Trace Elem. Med. Biol. 2019, 54, 226-231. [CrossRef] [PubMed] 
36. Rahimi, M.; Jafari, O.; Mohammdifar, A. Intensification of Liquid-Liquid Mass Transfer in Micromixer Assisted by Ultrasound Irradiation and $\mathrm{Fe}_{3} \mathrm{O}_{4}$ Nanoparticles. Chem. Eng. Process. Process Intensif. 2017, 111, 79-88. [CrossRef]

37. Zhang, Z.; Chen, K.; Zhao, Q.; Huang, M.; Ouyang, X. Comparative Adsorption of Heavy Metal Ions in Wastewater on Monolayer Molybdenum Disulfide. Green Energy Env. 2020, 6, 751-758. [CrossRef]

38. Tchounwou, P.B.; Yedjou, C.G.; Patlolla, A.K.; Sutton, D.J. Molecular, Clinical and Environmental Toxicicology Volume 3: Environmental Toxicology. Mol. Clin. Environ. Toxicol. 2012, 101, 133-164. [CrossRef]

39. Qiao, Y.; Yang, Y.; Gu, J.; Zhao, J. Distribution and Geochemical Speciation of Heavy Metals in Sediments from Coastal Area Suffered Rapid Urbanization, a Case Study of Shantou Bay, China. Mar. Pollut. Bull. 2013, 68, 140-146. [CrossRef]

40. Singh, R.; Singh, S.; Parihar, P.; Singh, V.P.; Prasad, S.M. Arsenic Contamination, Consequences and Remediation Techniques: A Review. Ecotoxicol. Environ. Saf. 2015, 112, 247-270. [CrossRef] [PubMed]

41. Gautam, R.K.; Sharma, S.K.; Mahiya, S.; Chattopadhyaya, M.C. Contamination of Heavy Metals in Aquatic Media: Transport, Toxicity and Technologies for Remediation. In Heavy Metals In Water: Presence, Removal and Safety Heavy; Sharma, S.K., Ed.; Royal Society of Chemistry: London, UK, 2014; pp. 1-24. [CrossRef]

42. Valko, M.; Morris, H.; Cronin, T.D.M. Metals, Toxicity and Oxidative Stress Metals. Curr. Med. Chem. 2005, 12, 1161-1208. [CrossRef]

43. Schroeder, H.; Duester, L.; Fabricius, A.L.; Ecker, D.; Breitung, V.; Ternes, T. ASediment Water (Interface) Mobility of Metal(Loid)s and Nutrients under Undisturbed Conditions and during Resuspension. J. Hazard. Mater. 2020, 394, 122543. [CrossRef] [PubMed]

44. Bhateria, R.; Singh, R. A Review on Nanotechnological Application of Magnetic Iron Oxides for Heavy Metal Removal. J. Water Process Eng. 2019, 31, 100845. [CrossRef]

45. Sonone, S.S.; Jadhav, S.V.; Sankhla, M.S.; Kumar, R. Water Contamination by Heavy Metals and Their Toxic Effect on Aquaculture and Human Health through Food Chain. Lett. Appl. NanoBioScience. 2020, 10, 2148-2166. [CrossRef]

46. Bradl, H.B. Sources and Origins of Heavy Metals. In Interface Science and Technology; Bradl, H.B., Ed.; Elsevier: Amsterdam, The Netherlands, 2005; pp. 1-27. [CrossRef]

47. Kyzas, G.Z.; Bomis, G.; Kosheleva, R.I.; Efthimiadou, E.K.; Favvas, E.P.; Kostoglou, M.; Mitropoulos, A.C. Nanobubbles Effect on Heavy Metal Ions Adsorption by Activated Carbon. Chem. Eng. J. 2019, 356, 91-97. [CrossRef]

48. Liosis, C.; Karvelas, E.G.; Karakasidis, T.; Sarris, I.E. Numerical Study of Magnetic Particles Mixing in Waste Water under an External Magnetic Field. J. Water Supply Res. Technol. 2020, 69, 266-275. [CrossRef]

49. Parvin, F.; Rikta, S.Y.; Tareq, S.M. Application of Nanomaterials for the Removal of Heavy Metal From Wastewater. In Nanotechnology in Water and Wastewater Treatment Theory and Applications Micro and Nano Technologies; Ahsan, A., Ismail, A.F., Eds.; Elsevier: Amsterdam, The Netherlands, 2019; pp. 137-157. [CrossRef]

50. Karvelas, E.; Liosis, C.; Karakasidis, T.; Sarris, I. Mixing of Particles in Micromixers under Different Angles and Velocities of the Incoming Water. Proceedings 2018, 2, 577. [CrossRef]

51. Karvelas, E.; Liosis, C.; Benos, L.; Karakasidis, T.; Sarris, I. Micromixing Efficiency of Particles in Heavy Metal Removal Processes under Various Inlet Conditions. Water 2019, 11, 1135. [CrossRef]

52. Wu, Y.; Pang, H.; Liu, Y.; Wang, X.; Yu, S.; Fu, D.; Chen, J.; Wang, X. Environmental Remediation of Heavy Metal Ions by Novel-Nanomaterials: A Review. Environ. Pollut. 2019, 246, 608-620. [CrossRef] [PubMed]

53. Zhu, L.; Li, C.; Wang, J.; Zhang, H.; Zhang, J.; Shen, Y.; Li, C.; Wang, C.; Xie, A. A Simple Method to Synthesize Modified Fe ${ }_{3} \mathrm{O}_{4}$ for the Removal of Organic Pollutants on Water Surface. Appl. Surf. Sci. 2012, 258, 6326-6330. [CrossRef]

54. Turan, N.B.; Erkan, H.S.; Engin, G.O.; Bilgili, M.S. Nanoparticles in the Aquatic Environment: Usage, Properties, Transformation and Toxicity-A Review. Process. Saf. Environ. Prot. 2019, 130, 238-249. [CrossRef]

55. Nowack, B.; Bucheli, T.D. Occurrence, Behavior and Effects of Nanoparticles in the Environment. Environ. Pollut. 2007, 150, 5-22. [CrossRef]

56. Gatoo, M.A.; Naseem, S.; Arfat, M.Y.; Mahmood, D.A.; Qasim, K.; Zubair, S. Physicochemical Properties of Nanomaterials: Implication in Associated Toxic Manifestations. Biomed Res. Int. 2014, 2014. [CrossRef]

57. Zhang, J.; Wu, L.; Chan, H.K.; Watanabe, W. Formation, Characterization, and Fate of Inhaled Drug Nanoparticles. Adv. Drug Deliv. Rev. 2011, 63, 441-455. [CrossRef] [PubMed]

58. Wadhawan, S.; Jain, A.; Nayyar, J.; Mehta, S.K. Role of Nanomaterials as Adsorbents in Heavy Metal Ion Removal from Waste Water: A Review. J. Water Process Eng. 2020, 33, 101038. [CrossRef]

59. Karvelas, E.; Liosis, C.; Karakasidis, T.; Sarris, I. Micromixing Nanoparticles and Contaminated Water Under Different Velocities for Optimum Heavy Metal Ions Adsorption. Env. Sci. Proc. 2020, 2, 65. [CrossRef]

60. Du, X.; He, J.; Zhu, J.; Sun, L.; An, S. Ag-Deposited Silica-Coated $\mathrm{Fe}_{3} \mathrm{O}_{4}$ Magnetic Nanoparticles Catalyzed Reduction of p-Nitrophenol. Appl. Surf. Sci. 2012, 258, 2717-2723. [CrossRef]

61. Mohammed, L.; Gomaa, H.G.; Ragab, D.; Zhu, J. Magnetic Nanoparticles for Environmental and Biomedical Applications: A Review. Particuology. 2017, 30, 1-14. [CrossRef]

62. Faraji, M.; Yamini, Y.; Saleh, A.; Rezaee, M.; Ghambarian, M.; Hassani, R. A Nanoparticle-Based Solid-Phase Extraction Procedure Followed by Flow Injection Inductively Coupled Plasma-Optical Emission Spectrometry to Determine Some Heavy Metal Ions in Water Samples. Anal. Chim. Acta 2010, 659, 172-177. [CrossRef]

63. Al-Ghouti, M.A.; Da'ana, D.A. Guidelines for the Use and Interpretation of Adsorption Isotherm Models: A Review. J. Hazard. Mater. 2020, 393, 122383. [CrossRef] [PubMed] 
64. Kurniawan, T.A.; Chan, G.Y.S.; Lo, W.H.; Babel, S. Physico-Chemical Treatment Techniques for Wastewater Laden with Heavy Metals. Chem. Eng. J. 2006, 118, 83-98. [CrossRef]

65. Wang, J.; Zhuang, S. Removal of Various Pollutants from Water and Wastewater by Modified Chitosan Adsorbents. Crit. Rev. Environ. Sci. Technol. 2017, 47, 2331-2386. [CrossRef]

66. Qu, X.; Alvarez, P.J.J.; Li, Q. Applications of Nanotechnology in Water and Wastewater Treatment. Water Res. 2013, 47, 3931-3946. [CrossRef]

67. Hu, H.; Xu, K. Physicochemical Technologies for HRPs and Risk Control. In High-Risk Pollutants in Wastewater; Hongqiang, R., Zhang, X., Eds.; Elsevier: Amsterdam, The Netherlands, 2019; pp. 169-207. [CrossRef]

68. Zhang, K.; Yong, F.; McCarthy, D.T.; Deletic, A. Predicting Long Term Removal of Heavy Metals from Porous Pavements for Stormwater Treatment. Water Res. 2018, 142, 236-245. [CrossRef]

69. Laurent, S.; Forge, D.; Port, M.; Roch, A.; Robic, C.; Vander Elst, L.; Muller, R.N. Magnetic Iron Oxide Nanoparticles: Synthesis, Stabilization, Vectorization, Physicochemical Characterizations and Biological Applications. Chem. Rev. 2008, 108, 2064-2110. [CrossRef]

70. Zia, M.; Phull, A.R.; Ali, J.S. Challenges of Iron Oxide Nanoparticles. Powder Technol. 2016, 7, 49-67.

71. Drmota, A.; Drofenik, M.; Koselj, J.; Žnidarši, A. Microemulsion Method for Synthesis of Magnetic Oxide Nanoparticles. In Microemulsions-An Introduction to Properties and Applications; Najjar, R., Ed.; InTech: Rijeka, Croatia, 2012; pp. $192-214$.

72. Ling, X.; Wei, L.; Hong, G.; Qiong, M. Synthesis and Characterization of Magnetic and Luminescent Fe $\mathrm{O}_{4} / \mathrm{CdTe}_{\mathrm{Nanocomposites}}$ Using Aspartic Acid as Linker. Chinese Chem. Lett. 2011, 22, 233-236. [CrossRef]

73. Takai, Z.; Mustafa, M.K.; Asman, S. Preparation and Characterization of Magnetite $\left(\mathrm{Fe}_{3} \mathrm{O}_{4}\right)$ Nanoparticles By Sol- Gel Method Preparation and Characterization of Magnetite $\left(\mathrm{Fe}_{3} \mathrm{O}_{4}\right)$ Nanoparticles By Sol-Gel Method. Asian J. Chem. 2018, 30, 2625-2630. [CrossRef]

74. Wang, Y.; Nkurikiyimfura, I.; Pan, Z. Sonochemical Synthesis of Magnetic Nanoparticles. Chem. Eng. Commun. 2014, 202, 616-621. [CrossRef]

75. Ge, S.; Shi, X.; Sun, K.; Li, C.; Uher, C.; Baker, J.R.; Holl, M.M.B.; Orr, B.G. Facile Hydrothermal Synthesis of Iron Oxide Nanoparticles with Tunable Magnetic Properties. J. Phys. Chem. 2009, 113, 13593-13599. [CrossRef]

76. Oćwieja, M.; Wegrzynowicz, A.; Maciejewska-Prończuk, J.; Michorczyk, P.; Adamczyk, Z.; Roman, M.; Bielańska, E. Preparation of Iron Oxide Nanoparticles Doped by Chromium for Application in Water-Gas Shift Reaction. Colloids Surf. A Physicochem. Eng. Asp. 2017, 523, 71-80. [CrossRef]

77. Compeán-Jasso, M.E.; Ruiz, F.; Martínez, J.R.; Herrera-Gómez, A. Magnetic Properties of Magnetite Nanoparticles Synthesized by Forced Hydrolysis. Mater. Lett. 2008, 62, 4248-4250. [CrossRef]

78. Stefanescu, M.; Stefanescu, O.; Stoia, M.; Lazau, C. Thermal Decomposition of Some Metal-Organic Precursors. J. Anal Calorim. 2007, 88, 27-32. [CrossRef]

79. Salazar-Alvarez, G.; Muhammed, M.; Zagorodni, A.A. Novel Flow Injection Synthesis of Iron Oxide Nanoparticles with Narrow Size Distribution. Chem. Eng. Sci. 2006, 61, 4625-4633. [CrossRef]

80. El-amin, M.F.; Saad, A.M.; Salama, A.; Sun, S. Modeling and Analysis of Magnetic Nanoparticles Injection in Water-Oil Two-Phase Flow in Porous Media under Magnetic Field Effect. Geofluids 2017, 2017, 12. [CrossRef]

81. Agostini, P.; Meffre, A.; Damien, L.L.; Benjamin, R. Electrospray Deposition of Isolated Chemically Synthesized Magnetic Nanoparticles. J. Nanopart Res. 2016, 18, 11. [CrossRef]

82. Atabaev, T.S.; Kim, H.K.; Hwang, Y.H. Fabrication of Bifunctional Core-Shell $\mathrm{Fe}_{3} \mathrm{O}_{4}$ Particles Coated with Ultrathin Phosphor Layer. Nanoscale Res. Lett. 2013, 8, 1-6. [CrossRef] [PubMed]

83. Rivera-Chaverra, M.J.; Restrepo-Parra, E.; Acosta-Medina, C.D.; Mello, A.; Ospina, R. Synthesis of Oxide Iron Nanoparticles Using Laser Ablation for Possible Hyperthermia Applications. Nanomaterials 2020, 10, 2099. [CrossRef]

84. Park, J.Y.; Lee, Y.J.; Khanna, P.K.; Jun, K.W.; Bae, J.W.; Kim, Y.H. Alumina-Supported Iron Oxide Nanoparticles as Fischer-Tropsch Catalysts: Effect of Particle Size of Iron Oxide. J. Mol. Catal. A Chem. 2010, 323, 84-90. [CrossRef]

85. Bokuniaeva, A.O.; Vorokh, A.S. Estimation of Particle Size Using the Debye Equation and the Scherrer Formula for Polyphasic $\mathrm{TiO}_{2}$ Powder. J. Phys. Conf. Ser. 2019, 1410, 12057. [CrossRef]

86. Mahdavi, M.; Ahmad, M.B.; Haron, M.J.; Namvar, F.; Nadi, B.; Ab Rahman, M.Z.; Amin, J. Synthesis, Surface Modification and Characterisation of Biocompatible Magnetic Iron Oxide Nanoparticles for Biomedical Applications. Molecules 2013, 18, 7533-7548. [CrossRef]

87. Alexander, L.; Klug, H.P. Determination of Crystallite Size with the X-Ray Spectrometer. J. Appl. Phys. 1950, 21, 137-142. [CrossRef]

88. Holzwarth, U.; Gibson, N. The Scherrer Equation versus the "Debye-Scherrer Equation". Nat. Nanotechnol. 2011, 6, 534. [CrossRef]

89. Zhu, N.; Ji, H.; Yu, P.; Niu, J.; Farooq, M.U.; Akram, M.W.; Udego, I.O.; Li, H.; Niu, X. Surface Modification of Magnetic Iron Oxide Nanoparticles. Nanomaterials 2018, 8, 810. [CrossRef] [PubMed]

90. Abu-dief, A.M.; Abdel-fatah, S.M. Development and Functionalization of Magnetic Nanoparticles as Powerful and Green Catalysts for Organic Synthesis. Beni-Suef Univ. J. Basic Appl. Sci. 2018, 7, 55-67. [CrossRef]

91. Jadhav, S.A.; Brunella, V.; Scalarone, D. Polymerizable Ligands as Stabilizers for Nanoparticles. Part. Part. Syst. Charact. 2014, 32, 417-428. [CrossRef] 
92. Cîrcu, M.; Nan, A.; Borodi, G.; Liebscher, J.; Turcu, R. Refinement of Magnetite Nanoparticles by Coating with Organic Stabilizers. Nanomaterials 2016, 6, 228. [CrossRef]

93. Sanchez, L.M.; Martin, D.A.; Alvarez, V.A.; Gonzalez, J.S. Polyacrylic Acid-Coated Iron Oxide Magnetic Nanoparticles: The Polymer Molecular Weight in Fl Uence. Colloids Surf. A 2018, 543, 28-37. [CrossRef]

94. Gholami, A.; Moghadassi, A.R.; Hosseini, S.M.; Shabani, S.; Gholami, F. Preparation and Characterization of Polyvinyl Chloride Based Nanocomposite Nanofiltration-Membrane Modified by Iron Oxide Nanoparticles for Lead Removal from Water. J. Ind. Eng. Chem. 2014, 20, 1517-1522. [CrossRef]

95. de Dios, A.S.; Díaz-García, M.E. Multifunctional Nanoparticles: Analytical Prospects. Anal. Chim. Acta. 2010, 666, 1-22. [CrossRef]

96. Karatapanis, A.E.; Petrakis, D.E.; Stalikas, C.D. A Layered Magnetic Iron/Iron Oxide Nanoscavenger for the Analytical Enrichment of ng- $\mathrm{L}^{-1}$ Concentration Levels of Heavy Metals from Water. Anal. Chim. Acta 2012, 726, 22-27. [CrossRef]

97. Ji, Y. Ions Removal by Iron Nanoparticles: A Study on Solid-Water Interface with Zeta Potential. Colloids Surf. A Physicochem. Eng. Asp. 2014, 444, 1-8. [CrossRef]

98. Kosmulski, M. PH-Dependent Surface Charging and Points of Zero Charge. IV. Update and New Approach. J. Colloid Interface Sci. 2009, 337, 439-448. [CrossRef] [PubMed]

99. Chavda, V.P. Nanobased Nano Drug Delivery: A Comprehensive Review. In Micro and Nano Technologies, Applications of Targeted Nano Drugs and Delivery Systems; Mohapatra, S., Ranjan, S., Dasgupta, N., Mishra, R.K., Thomas, S., Eds.; Elsevier: Amsterdam, The Netherlands, 2019; pp. 69-92. [CrossRef]

100. Crawford, R.J.; Harding, I.H.; Mainwaring, D.E. The Zeta Potential of Iron and Chromium Hydrous Oxides during Adsorption and Coprecipitation of Aqueous Heavy Metals. J. Colloid Interface Sci. 1996, 181, 561-570. [CrossRef]

101. Jiuhui, Q. Research progress of novel adsorption processes in water purification: A review. J. Environ. Sci. 2008, $20,1-13$. [CrossRef]

102. Petracic, O. Superparamagnetic Nanoparticle Ensembles. Superlattices Microstruct. 2010, 47, 569-578. [CrossRef]

103. Wu, W.; He, Q.; Jiang, C. Magnetic Iron Oxide Nanoparticles: Synthesis and Surface Functionalization Strategies. Nanoscale Res. Lett. 2008, 3, 397-415. [CrossRef]

104. Zhang, F.; Lan, J.; Zhao, Z.; Yang, Y.; Tan, R.; Song, W. Removal of Heavy Metal Ions from Aqueous Solution $\mathrm{Using}^{\mathrm{Fe}} \mathrm{O}_{4}-\mathrm{SiO}_{2}-$ Poly(1,2-Diaminobenzene) Core-Shell Sub-Micron Particles. J. Colloid Interface Sci. 2012, 387, 205-212. [CrossRef]

105. Fan, C.; Li, K.; Li, J.; Ying, D.; Wang, Y.; Jia, J. Comparative and Competitive Adsorption of Pb (II) and Cu (II) Using Tetraethylenepentamine Modified Chitosan/ $\mathrm{CoFe}_{2} \mathrm{O}_{4}$ Particles. J. Hazard. Mater. 2017, 326, 211-220. [CrossRef] [PubMed]

106. Amin, M.M.; Khodabakhshi, A. Removal of Cr (VI) From Simulated Electroplating. Environ. Eng. Manag. J. 2010, 9, 921-927.

107. Zhao, Y.G.; Shen, H.Y.; Pan, S.D.; Hu, M.Q. Synthesis, Characterization and Properties of Ethylenediamine-Functionalized Fe $\mathrm{O}_{4}$ Magnetic Polymers for Removal of Cr (VI) in Wastewater. J. Hazard. Mater. 2010, 182, 295-302. [CrossRef] [PubMed]

108. Kargin, D.B.; Konyukhov, Y.V.; Biseken, A.B.; Lileev, A.S.; Karpenkov, D.Y. Structure, Morphology and Magnetic Properties of Hematite and Maghemite Nanopowders Produced from Rolling Mill Scale. Steel Transl. 2020, 50, 151-158. [CrossRef]

109. Liu, Y.; Jia, S.; Wu, Q.; Ran, J.; Zhang, W.; Wu, S. Studies of $\mathrm{Fe}_{3} \mathrm{O}_{4}$-Chitosan Nanoparticles Prepared by Co-Precipitation under the Magnetic Field for Lipase Immobilization. Catal. Commun. 2011, 12, 717-720. [CrossRef]

110. Qiang, C.; Xu, J.; Zhang, Z.; Tian, L.; Xiao, S.; Liu, Y.; Xu, P. Magnetic Properties and Microwave Absorption Properties of Carbon Fibers Coated by $\mathrm{Fe}_{3} \mathrm{O}_{4}$ Nanoparticles. J. Alloy. Compd. 2010, 506, 93-97. [CrossRef]

111. Aredes, S.; Klein, B.; Pawlik, M. The Removal of Arsenic from Water Using Natural Iron Oxide Minerals. J. Clean. Prod. 2012, 29-30, 208-213. [CrossRef]

112. Rahim, P.S.; Abdul, R.A.A.; Wan, D.W.M.A. Review on the Application of Modified Iron Oxides as Heterogeneous Catalysts in Fenton Reactions. J. Clean. Prod. 2014, 64, 24-35. [CrossRef]

113. Xue, X.Y.; Cheng, R.; Shi, L.; Ma, Z.; Zheng, X. Nanomaterials for Water Pollution Monitoring and Remediation. Env. Chem. Lett. 2017, 15, 23-27. [CrossRef]

114. Tang, S.C.N.; Lo, I.M.C. Magnetic Nanoparticles: Essential Factors for Sustainable Environmental Applications. Water Res. 2013, 47, 2613-2632. [CrossRef] [PubMed]

115. Chowdhury, S.R.; Yanful, E.K. Arsenic and Chromium Removal by Mixed Magnetite-Maghemite Nanoparticles and the Effect of Phosphate on Removal. J. Env. Manag. 2010, 91, 2238-2247. [CrossRef] [PubMed]

116. Teja, A.S.; Koh, P.-Y. Synthesis, Properties, and Applications of Magnetic Iron Oxide Nanoparticles. Prog. Cryst. Growth Charact. Mater. 2009, 55, 22-45. [CrossRef]

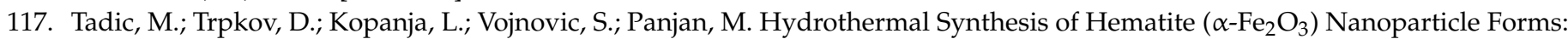
Synthesis Conditions, Structure, Particle Shape Analysis, Cytotoxicity and Magnetic Properties. J. Alloy. Compd. 2019, 792, 599-609. [CrossRef]

118. Gurmen, S.; Ebin, B. Production and Characterization of the Nanostructured Hollow Iron Oxide Spheres and Nanoparticles by Aerosol Route. J. Alloy. Compd. 2010, 492, 585-589. [CrossRef]

119. Tadic, M.; Kusigerski, V.; Markovic, D.; Milosevic, I.; Spasojevic, V. High Concentration of Hematite Nanoparticles in a Silica Matrix: Structural and Magnetic Properties. J. Magn. Magn. Mater. 2009, 321, 12-16. [CrossRef]

120. Estelrich, J.; Escribano, E.; Queralt, J.; Busquets, M.A. Iron Oxide Nanoparticles for Magnetically-Guided and MagneticallyResponsive Drug Delivery. Int. J. Mol. Sci. 2015, 16, 8070-8101. [CrossRef] 
121. Ling, D.; Lee, N.; Hyeon, T. Chemical Synthesis and Assembly of Uniformly Sized Iron Oxide Nanoparticles for Medical Applications. Acc. Chem. Res. 2015, 48, 1276-1285. [CrossRef] [PubMed]

122. Gholizadeh, A. A Comparative Study of Physical Properties in $\mathrm{Fe}_{3} \mathrm{O}_{4}$ Nanoparticles Prepared by Coprecipitation and Citrate Methods. J. Am. Ceram. Soc. 2017, 100, 3577-3588. [CrossRef]

123. Roto, R.; Yusran, Y.; Kuncaka, A. Magnetic Adsorbent of $\mathrm{Fe}_{3} \mathrm{O}_{4} @ \mathrm{SiO}_{2}$ Core-Shell Nanoparticles Modified with Thiol Group for Chloroauric Ion Adsorption. Appl. Surf. Sci. 2016, 377, 30-36. [CrossRef]

124. Wu, S.; Sun, A.; Zhai, F.; Wang, J.; Xu, W.; Zhang, Q.; Volinsky, A.A. $\mathrm{Fe}_{3} \mathrm{O}_{4}$ Magnetic Nanoparticles Synthesis from Tailings by Ultrasonic Chemical Co-Precipitation. Mater. Lett. 2011, 65, 1882-1884. [CrossRef]

125. Can, M.M.; Coşkun, M.; Firat, T. A Comparative Study of Nanosized Iron Oxide Particles; Magnetite $\left(\mathrm{Fe}_{3} \mathrm{O}_{4}\right)$, Maghemite $\left(\gamma-\mathrm{Fe}_{2} \mathrm{O}_{3}\right)$ and Hematite $\left(\alpha-\mathrm{Fe}_{2} \mathrm{O}_{3}\right)$, Using Ferromagnetic Resonance. J. Alloys Compd. 2012, 542, 241-247. [CrossRef]

126. Liang, X.; Xi, B.; Xiong, S.; Zhu, Y.; Xue, F.; Qian, Y. Porous Soft Magnetic Material: The Maghemite Microsphere with Hierarchical Nanoarchitecture and Its Application in Water Purification. Mater. Res. Bull. 2009, 44, 2233-2239. [CrossRef]

127. Mursalat, M.; Hastings, D.L.; Schoenitz, M.; Dreizin, E.L. Microspheres with Diverse Material Compositions Can Be Prepared by Mechanical Milling. Adv. Eng. Mater. 2020, 22, 1-4. [CrossRef]

128. Arbain, R.; Othman, M.; Palaniandy, S. Preparation of Iron Oxide Nanoparticles by Mechanical Milling. Miner. Eng. 2011, 24, 1-9. [CrossRef]

129. Nassar, N.N.; Hassan, A.; Carbognani, L.; Lopez-Linares, F.; Pereira-Almao, P. Iron Oxide Nanoparticles for Rapid Adsorption and Enhanced Catalytic Oxidation of Thermally Cracked Asphaltenes. Fuel 2012, 95, 257-262. [CrossRef]

130. Mohseni-Bandpi, A.; Al-Musawi, T.J.; Ghahramani, E.; Zarrabi, M.; Mohebi, S.; Vahed, S.A. Improvement of Zeolite Adsorption Capacity for Cephalexin by Coating with Magnetic $\mathrm{Fe}_{3} \mathrm{O}_{4}$ Nanoparticles. J. Mol. Liq. 2016, 218, 615-624. [CrossRef]

131. Wu, Y.W.; Zhang, J.; Liu, J.F.; Chen, L.; Deng, Z.L.; Han, M.X.; Wei, X.S.; Yu, A.M.; Zhang, H.L. Fe $\mathrm{O}_{4} @ \mathrm{ZrO}_{2} \mathrm{Nanoparticles}$ Magnetic Solid Phase Extraction Coupled with Flame Atomic Absorption Spectrometry for Chromium (III) Speciation in Environmental and Biological Samples. Appl. Surf. Sci. 2012, 258, 6772-6776. [CrossRef]

132. Mandel, K.; Hutter, F. The Magnetic Nanoparticle Separation Problem. Nano Today 2012, 7, 485-487. [CrossRef]

133. MacCuspie, R.I. Characterization of Nanomaterials for NanoEHS Studies. In Nanotechnology Environmental Health and Safety Risks, Regulation, and Management Micro and Nano Technologies, 3rd ed.; Hull, M.S., Bowman, D.M., Eds.; William Andrew Publishing: New York, NY, USA, 2018; pp. 59-82. [CrossRef]

134. Mourdikoudis, S.; Pallares, R.M.; Thanh, N.T.K. Characterization Techniques for Nanoparticles: Comparison and Complementarity upon Studying Nanoparticle Properties. Nanoscale 2018, 10, 12871-12934. [CrossRef] [PubMed]

135. Ivanova, E.P.; Bazaka, K.; Crawford, R.J. Metallic Biomaterials: Types and Advanced Applications. In New Functional Biomaterials for Medicine and Healthcare; Ivanova, E.P., Bazaka, K., Crawford, R.J., Eds.; Woodhead Publishing Limited: Sawston, UK, 2014; pp. 121-147. [CrossRef]

136. Fathy, M.M.; Fahmy, H.M.; Saad, O.A.; Elshemey, W.M. Silica-Coated Iron Oxide Nanoparticles as a Novel Nano-Radiosensitizer for Electron Therapy. Life Sci. 2019, 234, 116756. [CrossRef] [PubMed]

137. Koutsikou, T.S.; Krokidis, M.G.; Boukos, N.; Mitrikas, G.; Efthimiadou, E. Journal of Drug Delivery Science and Technology Synthesis, Characterization and Evaluation of Multi Sensitive Nanocarriers by Using the Layer by Layer Method. J. Drug Deliv. Sci. Technol. 2019, 53, 101142. [CrossRef]

138. Mitra, R.; Bhattacharya, S. Inhibition in Binding between Fullerene and a Bisporphyrin in Presence of Silver Nanoparticles in Solution: UV-Vis, DLS, SEM and TEM Studies. Spectrochim. Acta-Part A Mol. Biomol. Spectrosc. 2013, 114, 11-18. [CrossRef]

139. de la Calle, I.; Menta, M.; Klein, M.; Séby, F. Screening of $\mathrm{TiO}_{2}$ and Au Nanoparticles in Cosmetics and Determination of Elemental Impurities by Multiple Techniques (DLS, SP-ICP-MS, ICP-MS and ICP-OES). Talanta 2017, 171, 291-306. [CrossRef]

140. Yang, S.C.; Paik, S.Y.R.; Ryu, J.; Choi, K.O.; Kang, T.S.; Lee, J.K.; Song, C.W.; Ko, S. Dynamic Light Scattering-Based Method to Determine Primary Particle Size of Iron Oxide Nanoparticles in Simulated Gastrointestinal Fluid. Food Chem 2014, 161, 185-191. [CrossRef] [PubMed]

141. Zatsepin, A.F.; Kuznetsova, Y.A.; Sokolov, V.I. UV Absorption and Effects of Local Atomic Disordering in the Nickel Oxide Nanoparticles. J. Lumin. 2017, 183, 135-142. [CrossRef]

142. Tan, M.I.S.M.H.; Omar, A.F.; Rashid, M.; Hashim, U. VIS-NIR Spectral and Particles Distribution of Au, Ag, Cu, Al and Ni Nanoparticles Synthesized in Distilled Water Using Laser Ablation. Results Phys. 2019, 14, 102497. [CrossRef]

143. Kumar, A.; Dixit, C.K. Methods for Characterization of Nanoparticles. In Advances in Nanomedicine for the Delivery of Therapeutic Nucleic Acids; Nimesh, S., Chandra, R., Gupta, N., Eds.; Woodhead Publishing: Sawston, UK, 2017; pp. 43-48. [CrossRef]

144. Rouchota, M.; Loudos, G.; Efthimiadou, E.; Kordas, G.C.; Kagadis, G.C. Assessment of Low Energy X-Ray Imaging for Magnetic and Gold Nanoparticles. Phys. Medica 2016, 32, 257. [CrossRef]

145. Singh, A.K. Experimental Methodologies for the Characterization of Nanoparticles. In Engineered Nanoparticles; Hill-Parks, E., Ed.; Academic Press: San Diego, CA, USA, 2016; pp. 125-170. [CrossRef]

146. Li, X.; Li, Z.; Pan, D.; Xiang, Z.; Yoshimura, S.; Saito, H. Magnetization Behaviors of $\mathrm{Fe}_{3} \mathrm{O}_{4}$ Nanoparticles Studied by FrequencyModulated Magnetic Force Microscopy. Mater. Lett. 2014, 125, 36-39. [CrossRef]

147. Qiu, H.; Lv, L.; Pan, B.C.; Zhang, Q.J.; Zhang, W.M.; Zhang, Q.X. Critical Review in Adsorption Kinetic Models. J. Zhejiang Univ. Sci. A 2009, 10, 716-724. [CrossRef] 
148. Ashrafi, A.; Rahbar-Kelishami, A.; Shayesteh, H. Highly Efficient Simultaneous Ultrasonic Assisted Adsorption of Pb (II) by $\mathrm{Fe}_{3} \mathrm{O}_{4} @ \mathrm{MnO}_{2}$ Core-Shell Magnetic Nanoparticles: Synthesis and Characterization, Kinetic, Equilibrium, and Thermodynamic Studies. J. Mol. Struct. 2017, 1147, 40-47. [CrossRef]

149. Limousin, G.; Gaudet, J.P.; Charlet, L.; Szenknect, S.; Barthès, V.; Krimissa, M. Sorption Isotherms: A Review on Physical Bases, Modeling and Measurement. Appl. Geochem. 2007, 22, 249-275. [CrossRef]

150. Foo, K.Y.; Hameed, B.H. Insights into the Modeling of Adsorption Isotherm Systems. Chem. Eng. J. 2010, 156, 2-10. [CrossRef]

151. Xu, Q.; Wang, Y.; Jin, L.; Wang, Y.; Qin, M. Adsorption of Cu (II), Pb (II) and Cr (VI) from Aqueous Solutions Using Black Wattle Tannin-Immobilized Nanocellulose. J. Hazard. Mater. 2017, 339, 91-99. [CrossRef] [PubMed]

152. Sheet, I.; Kabbani, A.; Holail, H. Removal of Heavy Metals Using Nanostructured Graphite Oxide, Silica Nanoparticles and Silica/Graphite Oxide Composite. Energy Procedia 2014, 50, 130-138. [CrossRef]

153. Wang, X.; Cai, J.; Zhang, Y.; Li, L.; Jiang, L.; Wang, C. Heavy Metal Sorption Properties of Magnesium Titanate Mesoporous Nanorods. J. Mater. Chem. A 2015, 3, 11796-11800. [CrossRef]

154. Singh, A.K. Nanoparticle Ecotoxicology. In Engineered Nanoparticles; Hill-Parks, E., Ed.; Academic Press: San Diego, CA, USA, 2016; pp. 343-450. [CrossRef]

155. Altin, O.; Özbelge, H.Ö.; Doğu, T. Use of General Purpose Adsorption Isotherms for Heavy Metal-Clay Mineral Interactions. J. Colloid Interface Sci. 1998, 198, 130-140. [CrossRef]

156. Zhang, Y.; Ni, S.; Wang, X.; Zhang, W.; Lagerquist, L.; Qin, M.; Willför, S.; Xu, C.; Fatehi, P. Ultrafast Adsorption of Heavy Metal Ions onto Functionalized Lignin-Based Hybrid Magnetic Nanoparticles. Chem. Eng. J. 2019, 372, 82-91. [CrossRef]

157. Song, J.; Kong, H.; Jang, J. Adsorption of Heavy Metal Ions from Aqueous Solution by Polyrhodanine-Encapsulated Magnetic Nanoparticles. J. Colloid Interface Sci. 2011, 359, 505-511. [CrossRef] [PubMed]

158. Guo, X.; Wang, J. A General Kinetic Model for Adsorption: Theoretical Analysis and Modeling. J. Mol. Liq. 2019, 288, 111100. [CrossRef]

159. Dehghan, M.A.; Ghazanfari, M.H.; Jamialahmadi, M.; Helalizadeh, A. Adsorption of Silica Nanoparticles onto Calcite: Equilibrium, Kinetic, Thermodynamic and DLVO Analysis. Chem. Eng. J. 2015, 281, 334-344. [CrossRef]

160. Singh, D.; Verma, S.; Gautam, R.K.; Krishna, V. Copper Adsorption onto Synthesized Nitrilotriacetic Acid Functionalized Fe ${ }_{3} \mathrm{O}_{4}$ Nanoparticles: Kinetic, Equilibrium and Thermodynamic Studies. J. Environ. Chem. Eng. 2016, 3, 2161-2171. [CrossRef]

161. Shaker, M.A. Adsorption of Co (II), Ni (II) and Cu (II) Ions onto Chitosan-Modified Poly (Methacrylate) Nanoparticles: Dynamics, Equilibrium and Thermodynamics Studies. J. Taiwan Inst. Chem. Eng. 2015, 57, 111-122. [CrossRef]

162. Wang, J.; Guo, X. Adsorption Kinetic Models: Physical Meanings, Applications, and Solving Methods. J. Hazard. Mater. 2020, 390, 122156. [CrossRef]

163. Tanhaei, B.; Ayati, A.; Lahtinen, M.; Sillanpää, M. Preparation and Characterization of a Novel Chitosan $/ \mathrm{Al}_{2} \mathrm{O}_{3} / \mathrm{Magnetite}$ Nanoparticles Composite Adsorbent for Kinetic, Thermodynamic and Isotherm Studies of Methyl Orange Adsorption. Chem. Eng. J. 2015, 259, 1-10. [CrossRef]

164. Atar, N.; Olgun, A. Removal of Basic and Acid Dyes from Aqueous Solutions by a Waste Containing Boron Impurity. Desalination 2009, 249, 109-115. [CrossRef]

165. Mostafa, M.G.; Chen, Y.H.; Jean, J.S.; Liu, C.C.; Lee, Y.C. Kinetics and Mechanism of Arsenate Removal by Nanosized Iron Oxide-Coated Perlite. J. Hazard. Mater. 2011, 187, 89-95. [CrossRef]

166. Shaikh, M.N.; Helal, A.; Kalanthoden, A.N.; Najjar, B.; Abdul Aziz, M.; Mohamed, H.D. Sub-Nanometric Rh Decorated Magnetic Nanoparticles as Reusable Catalysts for Nitroarene Reduction in Water. Catal. Commun. 2019, 119, 134-138. [CrossRef]

167. Ain, Q.-U.; Farooq, M.U.; Jalees, M.I. Application of Magnetic Graphene Oxide for Water Purification: Heavy Metals Removal and Disinfection. J. Water Process Eng. 2020, 33, 101044. [CrossRef]

168. Nassar, N.N. Rapid Removal and Recovery of Pb (II) from Wastewater by Magnetic Nanoadsorbents. J. Hazard. Mater. 2010, 184, 538-546. [CrossRef] [PubMed]

169. Zhang, S.; Niu, H.; Cai, Y.; Zhao, X.; Shi, Y. Arsenite and Arsenate Adsorption on Coprecipitated Bimetal Oxide Magnetic Nanomaterials: $\mathrm{MnFe}_{2} \mathrm{O}_{4}$ and $\mathrm{CoFe}_{2} \mathrm{O}_{4}$. Chem. Eng. J. 2010, 158, 599-607. [CrossRef]

170. Kalantari, K.; Ahmad, M.B.; Fard Masoumi, H.R.; Shameli, K.; Basri, M.; Khandanlou, R. Rapid and High Capacity Adsorption of Heavy Metals by $\mathrm{Fe}_{3} \mathrm{O}_{4}$ /Montmorillonite Nanocomposite Using Response Surface Methodology: Preparation, Characterization, Optimization, Equilibrium Isotherms, and Adsorption Kinetics Study. J. Taiwan Inst. Chem. Eng. 2015, 49, 192-198. [CrossRef]

171. Hao, Y.M.; Man, C.; Hu, Z.B. Effective Removal of Cu (II) Ions from Aqueous Solution by Amino-Functionalized Magnetic Nanoparticles. J. Hazard. Mater. 2010, 184, 392-399. [CrossRef]

172. Wang, J.; Zheng, S.; Shao, Y.; Liu, J.; Xu, Z.; Zhu, D. Amino-Functionalized $\mathrm{Fe}_{3} \mathrm{O}_{4} @ \mathrm{SiO}_{2}$ Core-Shell Magnetic Nanomaterial as a Novel Adsorbent for Aqueous Heavy Metals Removal. J. Colloid Interface Sci. 2010, 349, 293-299. [CrossRef]

173. Ozmen, M.; Can, K.; Arslan, G.; Tor, A.; Cengeloglu, Y.; Ersoz, M. Adsorption of Cu (II) from Aqueous Solution by Using Modified $\mathrm{Fe}_{3} \mathrm{O}_{4}$ Magnetic Nanoparticles. Desalination 2010, 254, 162-169. [CrossRef]

174. Tran, H.V.; Tran, L.D.; Nguyen, T.N. Preparation of Chitosan/Magnetite Composite Beads and Their Application for Removal of $\mathrm{Pb}(\mathrm{II})$ and $\mathrm{Ni}(\mathrm{II})$ from Aqueous Solution. Mater. Sci. Eng. C 2010, 30, 304-310. [CrossRef]

175. Hu, H.; Wang, Z.; Pan, L. Synthesis of Monodisperse $\mathrm{Fe}_{3} \mathrm{O}_{4} @$ silica Core-Shell Microspheres and Their Application for Removal of Heavy Metal Ions from Water. J. Alloys Compd. 2010, 492, 656-661. [CrossRef] 
176. Sharma, Y.C.; Srivastava, V. Separation of Ni (II) Ions from Aqueous Solutions by Magnetic Nanoparticles. J. Chem. Eng. Data 2010, 55, 1441-1442. [CrossRef]

177. Zhao, Y.G.; Shen, H.Y.; Pan, S.D.; Hu, M.Q.; Xia, Q.H. Preparation and Characterization of Amino-Functionalized Nano-Fe ${ }_{3} \mathrm{O}_{4}$ Magnetic Polymer Adsorbents for Removal of Chromium (VI) Ions. J. Mater. Sci. 2010, 45, 5291-5301. [CrossRef]

178. Chowdhury, S.R.; Yanful, E.K.; Pratt, A.R. Arsenic Removal from Aqueous Solutions by Mixed Magnetite-Maghemite Nanoparticles. Environ. Earth Sci. 2011, 64, 411-423. [CrossRef]

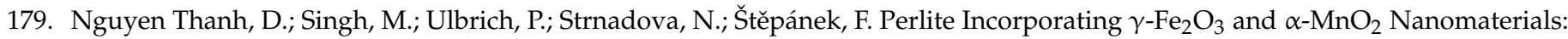
Preparation and Evaluation of a New Adsorbent for As (V) Removal. Sep. Purif. Technol. 2011, 82, 93-101. [CrossRef]

180. Hou, X.; Feng, J.; Liu, X.; Ren, Y.; Fan, Z.; Wei, T.; Meng, J.; Zhang, M. Synthesis of 3D Porous Ferromagnetic $\mathrm{NiFe}_{2} \mathrm{O}_{4}$ and Using as Novel Adsorbent to Treat Wastewater. J. Colloid Interface Sci. 2011, 362, 477-485. [CrossRef] [PubMed]

181. Xu, M.; Zhang, Y.; Zhang, Z.; Shen, Y.; Zhao, M.; Pan, G. Study on the Adsorption of $\mathrm{Ca}^{2+}, \mathrm{Cd}^{2+}$ and $\mathrm{Pb}^{2+}$ by $\mathrm{Magnetic} \mathrm{Fe}_{3} \mathrm{O}_{4}$ Yeast Treated with EDTA Dianhydride. Chem. Eng. J. 2011, 168, 737-745. [CrossRef]

182. Luo, X.; Luo, S.; Zhan, Y.; Shu, H.; Huang, Y.; Tu, X. Novel Cu (II) Magnetic Ion Imprinted Materials Prepared by Surface Imprinted Technique Combined with a Sol-Gel Process. J. Hazard. Mater. 2011, 192, 949-955. [CrossRef] [PubMed]

183. Panneerselvam, P.; Morad, N.; Tan, K.A. Magnetic Nanoparticle $\left(\mathrm{Fe}_{3} \mathrm{O}_{4}\right)$ Impregnated onto Tea Waste for the Removal of Nickel (II) from Aqueous Solution. J. Hazard. Mater. 2011, 186, 160-168. [CrossRef]

184. Pang, Y.; Zeng, G.; Tang, L.; Zhang, Y.; Liu, Y.; Lei, X.; Li, Z.; Zhang, J.; Liu, Z.; Xiong, Y. Preparation and Application of Stability Enhanced Magnetic Nanoparticles for Rapid Removal of Cr (VI). Chem. Eng. J. 2011, 175, 222-227. [CrossRef]

185. Bhaumik, M.; Maity, A.; Srinivasu, V.V.; Onyango, M.S. Enhanced Removal of Cr (VI) from Aqueous Solution Using Polypyrrole $/ \mathrm{Fe}_{3} \mathrm{O}_{4}$ Magnetic Nanocomposite. J. Hazard. Mater. 2011, 190, 381-390. [CrossRef]

186. Phuengprasop, T.; Sittiwong, J.; Unob, F. Removal of Heavy Metal Ions by Iron Oxide Coated Sewage Sludge. J. Hazard. Mater. 2011, 186, 502-507. [CrossRef]

187. Li, G.; Zhao, Z.; Liu, J.; Jiang, G. Effective Heavy Metal Removal from Aqueous Systems by Thiol Functionalized Magnetic Mesoporous Silica. J. Hazard. Mater. 2011, 192, 277-283. [CrossRef]

188. Sun, L.; Li, Y.; Sun, M.; Wang, H.; Xu, S.; Zhang, C.; Yang, Q. Porphyrin-Functionalized $\mathrm{Fe}_{3} \mathrm{O}_{4} @ \mathrm{SiO}_{2} \mathrm{Core}_{\mathrm{Shell} \mathrm{Magnetic}}$ Colorimetric Material for Detection, Adsorption and Removal of $\mathrm{Hg}^{2+}$ in Aqueous Solution. New J. Chem. 2011, 35, $2697-2704$. [CrossRef]

189. Hu, J.; Yang, S.; Wang, X. Adsorption of Cu (II) on $\beta$-Cyclodextrin Modified Multiwall Carbon Nanotube/Iron Oxides in the Absence/Presence of Fulvic Acid. J. Chem. Technol. Biotechnol. 2012, 87, 673-681. [CrossRef]

190. Paulino, A.T.; Belfiore, L.A.; Kubota, L.T.; Muniz, E.C.; Almeida, V.C.; Tambourgi, E.B. Effect of Magnetite on the Adsorption Behavior of $\mathrm{Pb}$ (II), Cd (II), and Cu (II) in Chitosan-Based Hydrogels. Desalination 2011, 275, 187-196. [CrossRef]

191. Gupta, V.K.; Agarwal, S.; Saleh, T.A. Chromium Removal by Combining the Magnetic Properties of Iron Oxide with Adsorption Properties of Carbon Nanotubes. Water Res. 2011, 45, 2207-2212. [CrossRef]

192. Wang, X.S.; Zhu, L.; Lu, H.J. Surface Chemical Properties and Adsorption of Cu (II) on Nanoscale Magnetite in Aqueous Solutions. Desalination 2011, 276, 154-160. [CrossRef]

193. Pang, Y.; Zeng, G.; Tang, L.; Zhang, Y.; Liu, Y.; Lei, X.; Li, Z.; Zhang, J.; Xie, G. PEI-Grafted Magnetic Porous Powder for Highly Effective Adsorption of Heavy Metal Ions. Desalination 2011, 281, 278-284. [CrossRef]

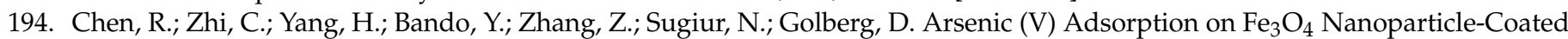
Boron Nitride Nanotubes. J. Colloid Interface Sci. 2011, 359, 261-268. [CrossRef]

195. Gong, J.; Wang, X.; Shao, X.; Yuan, S.; Yang, C.; Hu, X. Adsorption of Heavy Metal Ions by Hierarchically Structured MagnetiteCarbonaceous Spheres. Talanta 2012, 101, 45-52. [CrossRef] [PubMed]

196. Gong, J.L.; Wang, X.Y.; Zeng, G.M.; Chen, L.; Deng, J.H.; Zhang, X.R.; Niu, Q.Y. Copper (II) Removal by Pectin-Iron Oxide Magnetic Nanocomposite Adsorbent. Chem. Eng. J. 2012, 185-186, 100-107. [CrossRef]

197. Xu, P.; Zeng, G.M.; Huang, D.L.; Lai, C.; Zhao, M.H.; Wei, Z.; Li, N.J.; Huang, C.; Xie, G.X. Adsorption of Pb (II) by Iron Oxide Nanoparticles Immobilized Phanerochaete Chrysosporium: Equilibrium, Kinetic, Thermodynamic and Mechanisms Analysis. Chem. Eng. J. 2012, 203, 423-431. [CrossRef]

198. Xin, X.; Wei, Q.; Yang, J.; Yan, L.; Feng, R.; Chen, G.; Du, B.; Li, H. Highly Efficient Removal of Heavy Metal Ions by AmineFunctionalized Mesoporous $\mathrm{Fe}_{3} \mathrm{O}_{4}$ Nanoparticles. Chem. Eng. J. 2012, 184, 132-140. [CrossRef]

199. Ge, F.; Li, M.M.; Ye, H.; Zhao, B.X. Effective Removal of Heavy Metal Ions $\mathrm{Cd}^{2+}, \mathrm{Zn}^{2+}, \mathrm{Pb}^{2+}, \mathrm{Cu}^{2+}$ from Aqueous Solution by Polymer-Modified Magnetic Nanoparticles. J. Hazard. Mater. 2012, 211-212, 366-372. [CrossRef]

200. Feng, L.; Cao, M.; Ma, X.; Zhu, Y.; Hu, C. Superparamagnetic High-Surface-Area $\mathrm{Fe}_{3} \mathrm{O}_{4}$ Nanoparticles as Adsorbents for Arsenic Removal. J. Hazard. Mater. 2012, 217-218, 439-446. [CrossRef]

201. Ngomsik, A.F.; Bee, A.; Talbot, D.; Cote, G. Magnetic Solid-Liquid Extraction of Eu (III), La (III), Ni (II) and Co (II) with Maghemite Nanoparticles. Sep. Purif. Technol. 2012, 86, 1-8. [CrossRef]

202. Jin, Y.; Liu, F.; Tong, M.; Hou, Y. Removal of Arsenate by Cetyltrimethylammonium Bromide Modified Magnetic Nanoparticles. J. Hazard. Mater. 2012, 227-228, 461-468. [CrossRef] [PubMed]

203. Larraza, I.; López-Gónzalez, M.; Corrales, T.; Marcelo, G. Hybrid Materials: Magnetite-Polyethylenimine-Montmorillonite, as Magnetic Adsorbents for Cr (VI) Water Treatment. J. Colloid Interface Sci. 2012, 385, 24-33. [CrossRef] 
204. Zhang, C.; Sui, J.; Li, J.; Tang, Y.; Cai, W. Efficient Removal of Heavy Metal Ions by Thiol-Functionalized Superparamagnetic Carbon Nanotubes. Chem. Eng. J. 2012, 210, 45-52. [CrossRef]

205. Bhunia, P.; Kim, G.; Baik, C.; Lee, H. A Strategically Designed Porous Iron-Iron Oxide Matrix on Graphene for Heavy Metal Adsorption. Chem. Commun. 2012, 48, 9888-9890. [CrossRef] [PubMed]

206. Cho, D.W.; Jeon, B.H.; Chon, C.M.; Kim, Y.; Schwartz, F.W.; Lee, E.S.; Song, H. A Novel Chitosan/Clay/Magnetite Composite for Adsorption of Cu (II) and As (V). Chem. Eng. J. 2012, 200-202, 654-662. [CrossRef]

207. Wang, L.; Li, J.; Jiang, Q.; Zhao, L. Water-Soluble $\mathrm{Fe}_{3} \mathrm{O}_{4}$ Nanoparticles with High Solubility for Removal of Heavy-Metal Ions from Waste Water. Dalt. Trans. 2012, 41, 4544-4551. [CrossRef]

208. Hakami, O.; Zhang, Y.; Banks, C.J. Thiol-Functionalised Mesoporous Silica-Coated Magnetite Nanoparticles for High Efficiency Removal and Recovery of Hg from Water. Water Res. 2012, 46, 3913-3922. [CrossRef]

209. Parham, H.; Zargar, B.; Shiralipour, R. Fast and Efficient Removal of Mercury from Water Samples Using Magnetic Iron Oxide Nanoparticles Modified with 2-Mercaptobenzothiazole. J. Hazard. Mater. 2012, 205-206, 94-100. [CrossRef]

210. Luo, X.; Wang, C.; Luo, S.; Dong, R.; Tu, X.; Zeng, G. Adsorption of As(III) and As (V) from Water Using Magnetite Fe $\mathrm{O}_{4}$-Reduced Graphite Oxide- $\mathrm{MnO}_{2}$ Nanocomposites. Chem. Eng. J. 2012, 187, 45-52. [CrossRef]

211. Ji, L.; Zhou, L.; Bai, X.; Shao, Y.; Zhao, G.; Qu, Y.; Wang, C.; Li, Y. Facile Synthesis of Multiwall Carbon Nanotubes/Iron Oxides for Removal of Tetrabromobisphenol A and $\mathrm{Pb}$ (II). J. Mater. Chem. 2012, 22, 15853-15862. [CrossRef]

212. Roy, A.; Bhattacharya, J. Removal of $\mathrm{Cu}$ (II), Zn (II) and Pb (II) from Water Using Microwave-Assisted Synthesized Maghemite Nanotubes. Chem. Eng. J. 2012, 211-212, 493-500. [CrossRef]

213. Zhang, S.; Zhou, Y.F.; Nie, W.Y.; Song, L.Y. Preparation of $\mathrm{Fe}_{3} \mathrm{O}_{4} /$ Chitosan/Poly(Acrylic Acid) Composite Particles and Its Application in Adsorbing Copper Ion (II). Cellulose 2012, 19, 2081-2091. [CrossRef]

214. Mahdavi, M.; Ahmad, M.B.; Haron, M.J.; Gharayebi, Y.; Shameli, K.; Nadi, B. Fabrication and $\mathrm{Characterization} \mathrm{of} \mathrm{SiO}_{2} /(3-$ Aminopropyl)Triethoxysilane-Coated Magnetite Nanoparticles for Lead(II) Removal from Aqueous Solution. J. Inorg. Organomet. Polym. Mater. 2013, 23, 599-607. [CrossRef]

215. Mahapatra, A.; Mishra, B.G.; Hota, G. Electrospun $\mathrm{Fe}_{2} \mathrm{O}_{3}-\mathrm{Al}_{2} \mathrm{O}_{3}$ Nanocomposite Fibers as Efficient Adsorbent for Removal of Heavy Metal Ions from Aqueous Solution. J. Hazard. Mater. 2013, 258-259, 116-123. [CrossRef]

216. Ahmed, M.A.; Ali, S.M.; El-Dek, S.I.; Galal, A. Magnetite-Hematite Nanoparticles Prepared by Green Methods for Heavy Metal Ions Removal from Water. Mater. Sci. Eng. B 2013, 178, 744-751. [CrossRef]

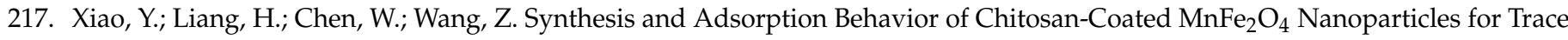
Heavy Metal Ions Removal. Appl. Surf. Sci. 2013, 285, 498-504. [CrossRef]

218. Chang, J.; Zhong, Z.; Xu, H.; Yao, Z.; Chen, R. Fabrication of Poly ( $\gamma$-Glutamic Acid)-Coated $\mathrm{Fe}_{3} \mathrm{O}_{4}$ Magnetic Nanoparticles and Their Application in Heavy Metal Removal. Chinese J. Chem. Eng. 2013, 21, 1244-1250. [CrossRef]

219. Badruddoza, A.Z.M.; Shawon, Z.B.Z.; Tay, W.J.D.; Hidajat, K.; Uddin, M.S. $\mathrm{Fe}_{3} \mathrm{O}_{4}$ /Cyclodextrin Polymer Nanocomposites for Selective Heavy Metals Removal from Industrial Wastewater. Carbohydr. Polym. 2013, 91, 322-332. [CrossRef]

220. Liang, H.; Xu, B.; Wang, Z. Self-Assembled 3D Flower-like $\alpha-\mathrm{Fe}_{2} \mathrm{O}_{3}$ Microstructures and Their Superior Capability for Heavy Metal Ion Removal. Mater. Chem. Phys. 2013, 141, 727-734. [CrossRef]

221. Karami, H. Heavy Metal Removal from Water by Magnetite Nanorods. Chem. Eng. J. 2013, 219, 209-216. [CrossRef]

222. Ren, Y.; Abbood, H.A.; He, F.; Peng, H.; Huang, K. Magnetic EDTA-Modified Chitosan $/ \mathrm{SiO}_{2} / \mathrm{Fe}_{3} \mathrm{O}_{4}$ Adsorbent: Preparation, Characterization, and Application in Heavy Metal Adsorption. Chem. Eng. J. 2013, 226, 300-311. [CrossRef]

223. Tang, Y.; Liang, S.; Wang, J.; Yu, S.; Wang, Y. Amino-Functionalized Core-Shell Magnetic Mesoporous Composite Microspheres for $\mathrm{Pb}$ (II) and Cd (II) Removal. J. Environ. Sci. 2013, 25, 830-837. [CrossRef]

224. Liu, M.; Wen, T.; Wu, X.; Chen, C.; Hu, J.; Li, J.; Wang, X. Synthesis of Porous $\mathrm{Fe}_{3} \mathrm{O}_{4}$ Hollow Microspheres/ Graphene Oxide Composite for Cr(vi) Removal. Dalt. Trans. 2013, 42, 14710-14717. [CrossRef]

225. Wei, Z.; Xing, R.; Zhang, X.; Liu, S.; Yu, H.; Li, P. Facile Template-Free Fabrication of Hollow Nestlike $\alpha$-Fe $\mathrm{O}_{3} \mathrm{O}_{3} \mathrm{Nanostructures}_{\text {for }}$ Water Treatment. ACS Appl. Mater. Interfaces 2013, 5, 598-604. [CrossRef]

226. Ren, T.; He, P.; Niu, W.; Wu, Y.; Ai, L.; Gou, X. Synthesis of $\alpha-\mathrm{Fe}_{2} \mathrm{O}_{3}$ Nanofibers for Applications in Removal and Recovery of Cr (VI) from Wastewater. Env. Sci. Pollut. Res. 2013, 20, 155-162. [CrossRef]

227. Zhang, J.; Zhai, S.; Li, S.; Xiao, Z.; Song, Y.; An, Q.; Tian, G. Pb (II) Removal of $\mathrm{Fe}_{3} \mathrm{O}_{4} @ \mathrm{SiO}_{2}-\mathrm{NH}_{2}$ Core-Shell Nanomaterials Prepared via a Controllable Sol-Gel Process. Chem. Eng. J. 2013, 215-216, 461-471. [CrossRef]

228. Burks, T.; Uheida, A.; Saleemi, M.; Eita, M.; Toprak, M.S.; Muhammed, M. Removal of Chromium (VI) Using Surface Modified Superparamagnetic Iron Oxide Nanoparticles. Sep. Sci. Technol. 2013, 48, 1243-1251. [CrossRef]

229. Zhao, L.; Chang, X.L.; Liao, R.; Zhang, X.; Xie, J.; Yu, B.; Wu, R.; Wang, R.; Yang, S.T. Facile Hydrothermal Preparation of S-Doped $\mathrm{Fe}_{3} \mathrm{O}_{4} @ \mathrm{C}$ Nanoparticles for $\mathrm{Cu}^{2+}$ Removal. Mater. Lett. 2014, 135, 154-157. [CrossRef]

230. Xu, Y.; Zhou, Y.; Li, R. Simultaneous Fluorescence Response and Adsorption of Functionalized $\mathrm{Fe}_{3} \mathrm{O}_{4} @ \mathrm{SiO}_{2} \mathrm{Nanoparticles} \mathrm{to}$ $\mathrm{Cd}^{2+}, \mathrm{Zn}^{2+}$ and $\mathrm{Cu}^{2+}$. Colloids Surf. A Physicochem. Eng. Asp. 2014, 459, 240-246. [CrossRef]

231. Feitoza, N.C.; Gonçalves, T.D.; Mesquita, J.J.; Menegucci, J.S.; Santos, M.K.M.S.; Chaker, J.A.; Cunha, R.B.; Medeiros, A.M.M.; Rubim, J.C.; Sousa, M.H. Fabrication of Glycine-Functionalized Maghemite Nanoparticles for Magnetic Removal of Copper from Wastewater. J. Hazard. Mater. 2014, 264, 153-160. [CrossRef] 
232. Ahmadi, A.; Heidarzadeh, S.; Mokhtari, A.R.; Darezereshki, E.; Harouni, H.A. Optimization of Heavy Metal Removal from Aqueous Solutions by Maghemite $\left(\gamma-\mathrm{Fe}_{2} \mathrm{O}_{3}\right)$ Nanoparticles Using Response Surface Methodology. J. Geochem. Explor. 2014, 147, 151-158. [CrossRef]

233. Hao, T.; Yang, C.; Rao, X.; Wang, J.; Niu, C.; Su, X. Facile Additive-Free Synthesis of Iron Oxide Nanoparticles for Efficient Adsorptive Removal of Congo Red and Cr (VI). Appl. Surf. Sci. 2014, 292, 174-180. [CrossRef]

234. Burks, T.; Avila, M.; Akhtar, F.; Göthelid, M.; Lansåker, P.C.; Toprak, M.S.; Muhammed, M.; Uheida, A. Studies on the Adsorption of Chromium (VI) onto 3-Mercaptopropionic Acid Coated Superparamagnetic Iron Oxide Nanoparticles. J. Colloid Interface Sci. 2014, 425, 36-43. [CrossRef]

235. Fang, X.B.; Fang, Z.Q.; Tsang, P.K.E.; Cheng, W.; Yan, X.M.; Zheng, L.C. Selective Adsorption of Cr (VI) from Aqueous Solution by EDA- $\mathrm{Fe}_{3} \mathrm{O}_{4}$ Nanoparticles Prepared from Steel Pickling Waste Liquor. Appl. Surf. Sci. 2014, 314, 655-662. [CrossRef]

236. Türk, T.; Alp, I. Arsenic Removal from Aqueous Solutions with Fe-Hydrotalcite Supported Magnetite Nanoparticle. J. Ind. Eng. Chem. 2014, 20, 732-738. [CrossRef]

237. Guo, X.; Du, B.; Wei, Q.; Yang, J.; Hu, L.; Yan, L.; Xu, W. Synthesis of Amino Functionalized Magnetic Graphenes Composite Material and Its Application to Remove $\mathrm{Cr}$ (VI), $\mathrm{Pb}$ (II), $\mathrm{Hg}$ (II), Cd (II) and Ni (II) from Contaminated Water. J. Hazard. Mater. 2014, 278, 211-220. [CrossRef] [PubMed]

238. Lan, S.; Leng, Z.; Guo, N.; Wu, X.; Gan, S. Sesbania Gum-Based Magnetic Carbonaceous Nanocomposites: Facile Fabrication and Adsorption Behavior. Colloids Surf. A Physicochem. Eng. Asp. 2014, 446, 163-171. [CrossRef]

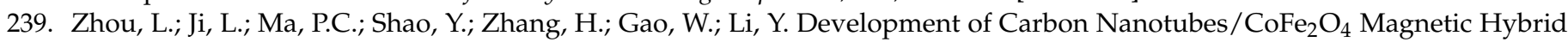
Material for Removal of Tetrabromobisphenol A and Pb (II). J. Hazard. Mater. 2014, 265, 104-114. [CrossRef]

240. Kalantari, K.; Ahmad, M.B.; Masoumi, H.R.F.; Shameli, K.; Basri, M.; Khandanlou, R. Rapid Adsorption of Heavy Metals by $\mathrm{Fe}_{3} \mathrm{O}_{4}$ /Talc Nanocomposite and Optimization Study Using Response Surface Methodology. Int. J. Mol. Sci. 2014, 15, 12913-12927. [CrossRef]

241. Lasheen, M.R.; El-Sherif, I.Y.; Sabry, D.Y.; El-Wakeel, S.T.; El-Shahat, M.F. Removal and Recovery of Cr (VI) by Magnetite Nanoparticles. Desalin. Water Treat. 2014, 52, 6464-6473. [CrossRef]

242. Zhang, Y.; Yan, L.; Xu, W.; Guo, X.; Cui, L.; Gao, L.; Wei, Q.; Du, B. Adsorption of Pb (II) and Hg (II) from Aqueous Solution Using Magnetic $\mathrm{CoFe}_{2} \mathrm{O}_{4}$-Reduced Graphene Oxide. J. Mol. Liq. 2014, 191, 177-182. [CrossRef]

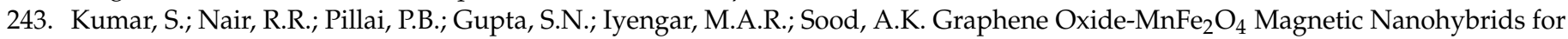
Efficient Removal of Lead and Arsenic from Water. ACS Appl. Mater. Interfaces 2014, 6, 17426-17436. [CrossRef]

244. Qi, X.; Li, N.; Xu, Q.; Chen, D.; Li, H.; Lu, J. Water-Soluble $\mathrm{Fe}_{3} \mathrm{O}_{4}$ Superparamagnetic Nanocomposites for the Removal of Low Concentration Mercury (II) Ions from Water. RSC Adv. 2014, 4, 47643-47648. [CrossRef]

245. Chen, Z.; Geng, Z.; Zhang, Z.; Ren, L.; Tao, T.; Yang, R.; Guo, Z. Synthesis of Magnetic $\mathrm{Fe}_{3} \mathrm{O}_{4} @ \mathrm{C}$ Nanoparticles Modified with $-\mathrm{SO}_{3} \mathrm{H}$ and $-\mathrm{COOH}$ Groups for Fast Removal of $\mathrm{Pb}^{2+}, \mathrm{Hg}^{2+}$, and $\mathrm{Cd}^{2+}$ Ions. Eur. J. Inorg. Chem. 2015, 2014, 3172-3177. [CrossRef]

246. Peng, X.; Xu, F.; Zhang, W.; Wang, J.; Zeng, C.; Niu, M.; Chmielewská, E. Magnetic $\mathrm{Fe}_{3} \mathrm{O}_{4} @$ @ Silica-Xanthan Gum Composites for Aqueous Removal and Recovery of $\mathrm{Pb}^{2+}$. Colloids Surf. A Physicochem. Eng. Asp. 2014, 443, 27-36. [CrossRef]

247. Luo, X.; Zeng, J.; Liu, S.; Zhang, L. An Effective and Recyclable Adsorbent for the Removal of Heavy Metal Ions from Aqueous System: Magnetic Chitosan/Cellulose Microspheres. Bioresour. Technol. 2015, 194, 403-406. [CrossRef] [PubMed]

248. Culita, D.C.; Simonescu, C.M.; Dragne, M.; Stanica, N.; Munteanu, C.; Preda, S.; Oprea, O. Effect of Surfactant Concentration on Textural, Morphological and Magnetic Properties of $\mathrm{CoFe}_{2} \mathrm{O}_{4}$ Nanoparticles and Evaluation of Their Adsorptive Capacity for Pb (II) Ions. Ceram. Int. 2015, 41, 13553-13560. [CrossRef]

249. Madrakian, T.; Afkhami, A.; Zadpour, B.; Ahmadi, M. New Synthetic Mercaptoethylamino Homopolymer-Modified Maghemite Nanoparticles for Effective Removal of Some Heavy Metal Ions from Aqueous Solution. J. Ind. Eng. Chem. 2015, 21, 1160-1166. [CrossRef]

250. Tadjarodi, A.; Abbaszadeh, A.; Taghizadeh, M.; Shekari, N.; Asgharinezhad, A.A. Solid Phase Extraction of Cd (II) and Pb (II) Ions Based on a Novel Functionalized $\mathrm{Fe}_{3} \mathrm{O}_{4} @ \mathrm{SiO}_{2}$ Core-Shell Nanoparticles with the Aid of Multivariate Optimization Methodology. Mater. Sci. Eng. C. 2015, 49, 416-421. [CrossRef] [PubMed]

251. Gautam, R.K.; Gautam, P.K.; Banerjee, S.; Soni, S.; Singh, S.K.; Chattopadhyaya, M.C. Removal of Ni (II) by Magnetic Nanoparticles. J. Mol. Liq. 2015, 204, 60-69. [CrossRef]

252. Souda, P.; Sreejith, L. Magnetic Hydrogel for Better Adsorption of Heavy Metals from Aqueous Solutions. J. Environ. Chem. Eng. 2015, 3, 1882-1891. [CrossRef]

253. Kumari, M.; Pittman, C.U.; Mohan, D. Heavy Metals [Chromium (VI) and Lead (II)] Removal from Water Using Mesoporous Magnetite $\left(\mathrm{Fe}_{3} \mathrm{O}_{4}\right)$ Nanospheres. J. Colloid Interface Sci. 2015, 442, 120-132. [CrossRef]

254. Morillo, D.; Uheida, A.; Pérez, G.; Muhammed, M.; Valiente, M. Arsenate Removal with 3-Mercaptopropanoic Acid-Coated Superparamagnetic Iron Oxide Nanoparticles. J. Colloid Interface Sci. 2015, 438, 227-234. [CrossRef]

255. Shan, C.; Ma, Z.; Tong, M. Efficient Removal of Free and Nitrilotriacetic Acid Complexed Cd (II) from Water by Poly(1Vinylimidazole)-Grafted $\mathrm{Fe}_{3} \mathrm{O}_{4} @ \mathrm{SiO}_{2}$ Magnetic Nanoparticles. J. Hazard. Mater. 2015, 299, 479-485. [CrossRef]

256. Chávez-Guajardo, A.E.; Medina-Llamas, J.C.; Maqueira, L.; Andrade, C.A.S.; Alves, K.G.B.; de Melo, C.P. Efficient Removal of $\mathrm{Cr}$ (VI) and $\mathrm{Cu}$ (II) Ions from Aqueous Media by Use of Polypyrrole/Maghemite and Polyaniline/Maghemite Magnetic Nanocomposites. Chem. Eng. J. 2015, 281, 826-836. [CrossRef] 
257. Cui, L.; Wang, Y.; Gao, L.; Hu, L.; Yan, L.; Wei, Q.; Du, B. EDTA Functionalized Magnetic Graphene Oxide for Removal of Pb (II), $\mathrm{Hg}$ (II) and Cu (II) in Water Treatment: Adsorption Mechanism and Separation Property. Chem. Eng. J. 2015, 281, 1-10. [CrossRef]

258. Moradinasab, S.; Behzad, M. Removal of Heavy Metals from Aqueous Solution Using $\mathrm{Fe}_{3} \mathrm{O}_{4}$ Nanoparticles Coated with Schiff Base Ligand. Desalin. Water Treat. 2016, 57, 4028-4036. [CrossRef]

259. Mittal, A.; Ahmad, R.; Hasan, I. Poly (Methyl Methacrylate)-Grafted Alginate $/ \mathrm{Fe}_{3} \mathrm{O}_{4}$ Nanocomposite: Synthesis and Its Application for the Removal of Heavy Metal Ions. Desalin. Water Treat. 2016, 57, 19820-19833. [CrossRef]

260. Zhang, X.; Wu, T.; Zhang, Y.; Ng, D.H.L.; Zhao, H.; Wang, G. Adsorption of $\mathrm{Hg}^{2+}$ by Thiol Functionalized Hollow Mesoporous Silica Microspheres with Magnetic Cores. RSC Adv. 2015, 5, 51446-51453. [CrossRef]

261. Wang, H.; Yuan, X.; Wu, Y.; Chen, X.; Leng, L.; Wang, H.; Li, H.; Zeng, G. Facile Synthesis of Polypyrrole Decorated Reduced Graphene Oxide- $\mathrm{Fe}_{3} \mathrm{O}_{4}$ Magnetic Composites and Its Application for the Cr (VI) Removal. Chem. Eng. J. 2015, 262, 597-606. [CrossRef]

262. Duan, S.; Tang, R.; Xue, Z.; Zhang, X.; Zhao, Y.; Zhang, W.; Zhang, J.; Wang, B.; Zeng, S.; Sun, D. Effective Removal of Pb (II) Using Magnetic $\mathrm{Co}_{0.6} \mathrm{Fe}_{2.4} \mathrm{O}_{4}$ Micro-Particles as the Adsorbent: Synthesis and Study on the Kinetic and Thermodynamic Behaviors for Its Adsorption. Colloids Surf. A Physicochem. Eng. Asp. 2015, 469, 211-223. [CrossRef]

263. Shan, R.R.; Yan, L.G.; Yang, K.; Hao, Y.F.; Du, B. Adsorption of Cd (II) by Mg-Al- $\mathrm{CO}_{3}$ - and Magnetic $\mathrm{Fe}_{3} \mathrm{O}_{4} / \mathrm{Mg}-\mathrm{Al}-\mathrm{CO}$-Layered Double Hydroxides: Kinetic, Isothermal, Thermodynamic and Mechanistic Studies. J. Hazard. Mater 2015, 299, 42-49. [CrossRef]

264. Lasheen, M.R.; El-Sherif, I.Y.; Tawfik, M.E.; El-Wakeel, S.T.; El-Shahat, M.F. Preparation and Adsorption Properties of Nano Magnetite Chitosan Films for Heavy Metal Ions from Aqueous Solution. Mater. Res. Bull. 2016, 80, 344-350. [CrossRef]

265. Bao, S.; Tang, L.; Li, K.; Ning, P.; Peng, J.; Guo, H.; Zhu, T.; Liu, Y. Highly Selective Removal of Zn (II) Ion from Hot-Dip Galvanizing Pickling Waste with Amino-Functionalized $\mathrm{Fe}_{3} \mathrm{O}_{4} @ \mathrm{SiO}_{2}$ Magnetic Nano-Adsorbent. J. Colloid Interface Sci. 2016, 462, 235-242. [CrossRef] [PubMed]

266. Tamez, C.; Hernandez, R.; Parsons, J.G. Removal of Cu (II) and Pb (II) from Aqueous Solution Using Engineered Iron Oxide Nanoparticles. Microchem. J. 2016, 125, 97-104. [CrossRef]

267. Babu, C.M.; Vinodh, R.; Sundaravel, B.; Abidov, A.; Peng, M.M.; Cha, W.S.; Jang, H.T. Characterization of Reduced Graphene Oxide Supported Mesoporous $\mathrm{Fe}_{2} \mathrm{O}_{3} / \mathrm{TiO}_{2}$ Nanoparticles and Adsorption of As (III) and As (V) from Potable Water. J. Taiwan Inst. Chem. Eng. 2016, 62, 199-208. [CrossRef]

268. Wang, Z.; Xu, J.; Hu, Y.; Zhao, H.; Zhou, J.; Liu, Y.; Lou, Z.; Xu, X. Functional Nanomaterials: Study on Aqueous Hg (II) Adsorption by Magnetic $\mathrm{Fe}_{3} \mathrm{O}_{4} @ \mathrm{SiO}_{2}-\mathrm{SH}$ Nanoparticles. J. Taiwan Inst. Chem. Eng. 2016, 60, 394-402. [CrossRef]

269. Singh, D.; Singh, S.K.; Atar, N.; Krishna, V. Amino Acid Functionalized Magnetic Nanoparticles for Removal of Ni (II) from Aqueous Solution. J. Taiwan Inst. Chem. Eng. 2016, 67, 148-160. [CrossRef]

270. Davarnejad, R.; Panahi, P. Cu (II) Removal from Aqueous Wastewaters by Adsorption on the Modified $\mathrm{Henna}$ with $\mathrm{Fe}_{3} \mathrm{O}_{4}$ Nanoparticles Using Response Surface Methodology. Sep. Purif. Technol. 2016, 158, 286-292. [CrossRef]

271. Yan, L.; Li, S.; Yu, H.; Shan, R.; Du, B.; Liu, T. Facile Solvothermal Synthesis of $\mathrm{Fe}_{3} \mathrm{O}_{4}$ / Bentonite for Efficient Removal of Heavy Metals from Aqueous Solution. Powder Technol. 2016, 301, 632-640. [CrossRef]

272. Bagbi, Y.; Sarswat, A.; Mohan, D.; Pandey, A.; Solanki, P.R. Lead ( $\left.\mathrm{Pb}^{2+}\right)$ Adsorption by Monodispersed Magnetite Nanoparticles: Surface Analysis and Effects of Solution Chemistry. J. Environ. Chem. Eng. 2016, 4, 4237-4247. [CrossRef]

273. Rajput, S.; Pittman, C.U.; Mohan, D. Magnetic Magnetite $\left(\mathrm{Fe}_{3} \mathrm{O}_{4}\right)$ Nanoparticle Synthesis and Applications for Lead $\left(\mathrm{Pb}^{2+}\right)$ and Chromium $\left(\mathrm{Cr}^{6+}\right)$ Removal from Water. J. Colloid Interface Sci. 2016, 468, 334-346. [CrossRef] [PubMed]

274. Zhu, X.; Song, T.; Lv, Z.; Ji, G. High-Efficiency and Low-Cost $\alpha-\mathrm{Fe}_{2} \mathrm{O}_{3}$ Nanoparticles-Coated Volcanic Rock for Cd (II) Removal from Wastewater. Process. Saf. Environ. Prot. 2016, 104, 373-381. [CrossRef]

275. Danesh, N.; Hosseini, M.; Ghorbani, M.; Marjani, A. Fabrication, Characterization and Physical Properties of a Novel Magnetite Graphene Oxide/Lauric Acid Nanoparticles Modified by Ethylenediaminetetraacetic Acid and Its Applications as an Adsorbent for the Removal of $\mathrm{Pb}$ (II) Ions. Synth. Met. 2016, 220, 508-523. [CrossRef]

276. Gao, J.; He, Y.; Zhao, X.; Ran, X.; Wu, Y.; Su, Y.; Dai, J. Single Step Synthesis of Amine-Functionalized Mesoporous Magnetite Nanoparticles and Their Application for Copper Ions Removal from Aqueous Solution. J. Colloid Interface Sci. 2016, 481, 220-228. [CrossRef]

277. Habila, M.A.; Alothman, Z.A.; El-Toni, A.M.; Labis, J.P.; Soylak, M. Synthesis and Application of $\mathrm{Fe}_{3} \mathrm{O}_{4} @ S i \mathrm{~S}_{2} @ \mathrm{TiO} \mathrm{O}_{2}$ for Photocatalytic Decomposition of Organic Matrix Simultaneously with Magnetic Solid Phase Extraction of Heavy Metals Prior to ICP-MS Analysis. Talanta 2016, 154, 539-547. [CrossRef]

278. Khandanlou, R.; Fard Masoumi, H.R.; Ahmad, M.B.; Shameli, K.; Basri, M.; Kalantari, K. Enhancement of Heavy Metals Sorption via Nanocomposites of Rice Straw and $\mathrm{Fe}_{3} \mathrm{O}_{4}$ Nanoparticles Using Artificial Neural Network (ANN). Ecol. Eng. 2016, 91, 249-256. [CrossRef]

279. Fan, H.L.; Li, L.; Zhou, S.F.; Liu, Y.Z. Continuous Preparation of $\mathrm{Fe}_{3} \mathrm{O}_{4}$ Nanoparticles Combined with Surface Modification by L-Cysteine and Their Application in Heavy Metal Adsorption. Ceram. Int. 2016, 42, 4228-4237. [CrossRef]

280. Mahmoud, M.E.; Amira, M.F.; Zaghloul, A.A.; Ibrahim, G.A.A. High Performance Microwave-Enforced Solid Phase Extraction of Heavy Metals from Aqueous Solutions Using Magnetic Iron Oxide Nanoparticles-Protected-Nanosilica. Sep. Purif. Technol. 2016, 163, 169-172. [CrossRef]

281. Mukherjee, D.; Ghosh, S.; Majumdar, S.; Annapurna, K. Green Synthesis of $\alpha-\mathrm{Fe}_{2} \mathrm{O}_{3}$ Nanoparticles for Arsenic (V) Remediation with a Novel Aspect for Sludge Management. J. Environ. Chem. Eng. 2016, 4, 639-650. [CrossRef] 
282. Zhao, J.; Liu, J.; Li, N.; Wang, W.; Nan, J.; Zhao, Z.; Cui, F. Highly Efficient Removal of Bivalent Heavy Metals from Aqueous Systems by Magnetic Porous $\mathrm{Fe}_{3} \mathrm{O}_{4}-\mathrm{MnO}_{2}$ : Adsorption Behavior and Process Study. Chem. Eng. J. 2016, 304, 737-746. [CrossRef]

283. Liu, Y.; Fu, R.; Sun, Y.; Zhou, X.; Baig, S.A.; Xu, X. Multifunctional Nanocomposites $\mathrm{Fe}_{3} \mathrm{O}_{4} @ S i \mathrm{O}_{2}$-EDTA for Pb (II) and Cu (II) Removal from Aqueous Solutions. Appl. Surf. Sci. 2016, 369, 267-276. [CrossRef]

284. Shen, W.; $\mathrm{Mu}, \mathrm{Y}$.; Xiao, T.; Ai, Z. Magnetic $\mathrm{Fe}_{3} \mathrm{O}_{4}-\mathrm{FeB}$ Nanocomposites with Promoted $\mathrm{Cr}$ (VI) Removal Performance. Chem. Eng. J. 2016, 285, 57-68. [CrossRef]

285. Beduk, F. Superparamagnetic Nanomaterial $\mathrm{Fe}_{3} \mathrm{O}_{4}-\mathrm{TiO}_{2}$ for the Removal of As (V) and As (III) from Aqueous Solutions. Env. Technol. 2016, 37, 1790-1801. [CrossRef] [PubMed]

286. Beheshti, H.; Irani, M.; Hosseini, L.; Rahimi, A.; Aliabadi, M. Removal of Cr (VI) from Aqueous Solutions Using Chitosan/MWCNT/ $\mathrm{Fe}_{3} \mathrm{O}_{4}$ Composite Nanofibers-Batch and Column Studies. Chem. Eng. J. 2016, 284, 557-564. [CrossRef]

287. Zhao, D.; Gao, X.; Wu, C.; Xie, R.; Feng, S.; Chen, C. Facile Preparation of Amino Functionalized Graphene Oxide Decorated with $\mathrm{Fe}_{3} \mathrm{O}_{4}$ Nanoparticles for the Adsorption of Cr (VI). Appl. Surf. Sci. 2016, 384, 1-9. [CrossRef]

288. Chen, K.; He, J.; Li, Y.; Cai, X.; Zhang, K.; Liu, T.; Hu, Y.; Lin, D.; Kong, L.; Liu, J. Removal of Cadmium and Lead Ions from Water by Sulfonated Magnetic Nanoparticle Adsorbents. J. Colloid Interface Sci. 2017, 494, 307-316. [CrossRef] [PubMed]

289. Hasanzadeh, R.; Moghadam, P.N.; Bahri-Laleh, N.; Sillanpää, M. Effective Removal of Toxic Metal Ions from Aqueous Solutions:

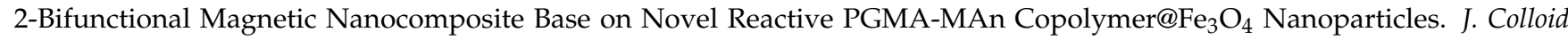
Interface Sci. 2017, 490, 727-746. [CrossRef] [PubMed]

290. Elfeky, S.A.; Mahmoud, S.E.; Youssef, A.F. Applications of CTAB Modified Magnetic Nanoparticles for Removal of Chromium (VI) from Contaminated Water. J. Adv. Res. 2017, 8, 435-443. [CrossRef] [PubMed]

291. Ke, F.; Jiang, J.; Li, Y.; Liang, J.; Wan, X.; Ko, S. Highly Selective Removal of $\mathrm{Hg}^{2+}$ and $\mathrm{Pb}^{2+}$ by Thiol-Functionalized Fe $3 \mathrm{O}_{4} @$ metalOrganic Framework Core-Shell Magnetic Microspheres. Appl. Surf. Sci. 2017, 413, 266-274. [CrossRef]

292. Guo, S.; Jiao, P.; Dan, Z.; Duan, N.; Zhang, J.; Chen, G.; Gao, W. Synthesis of Magnetic Bioadsorbent for Adsorption of Zn (II), Cd (II) and $\mathrm{Pb}$ (II) Ions from Aqueous Solution. Chem. Eng. Res. Des. 2017, 126, 217-231. [CrossRef]

293. Jin, S.; Park, B.C.; Ham, W.S.; Pan, L.; Kim, Y.K. Effect of the Magnetic Core Size of Amino-Functionalized $\mathrm{Fe}_{3} \mathrm{O}_{4}-\mathrm{Mesoporous}^{-}$ $\mathrm{SiO}_{2}$ Core-Shell Nanoparticles on the Removal of Heavy Metal Ions. Colloids Surfaces A Physicochem. Eng. Asp. 2017, 531, 133-140. [CrossRef]

294. Ahmadi, M.; Hazrati Niari, M.; Kakavandi, B. Development of Maghemite Nanoparticles Supported on Cross-Linked Chitosan ( $\left.\gamma-\mathrm{Fe}_{2} \mathrm{O}_{3} @ \mathrm{CS}\right)$ as a Recoverable Mesoporous Magnetic Composite for Effective Heavy Metals Removal. J. Mol. Liq. 2017, 248, 184-196. [CrossRef]

295. Bagbi, Y.; Sarswat, A.; Mohan, D.; Pandey, A.; Solanki, P.R. Lead and Chromium Adsorption from Water Using L-Cysteine Functionalized Magnetite $\left(\mathrm{Fe}_{3} \mathrm{O}_{4}\right)$ Nanoparticles. Sci. Rep. 2017, 7, 1-15. [CrossRef]

296. Jiryaei Sharahi, F.; Shahbazi, A. Melamine-Based Dendrimer Amine-Modified Magnetic Nanoparticles as an Efficient Pb (II) Adsorbent for Wastewater Treatment: Adsorption Optimization by Response Surface Methodology. Chemosphere 2017, 189, 291-300. [CrossRef]

297. Sun, T.; Zhao, Z.; Liang, Z.; Liu, J.; Shi, W.; Cui, F. Efficient Removal of Arsenite through Photocatalytic Oxidation and Adsorption by $\mathrm{ZrO}_{2}-\mathrm{Fe}_{3} \mathrm{O}_{4}$ Magnetic Nanoparticles. Appl. Surf. Sci. 2017, 416, 656-665. [CrossRef]

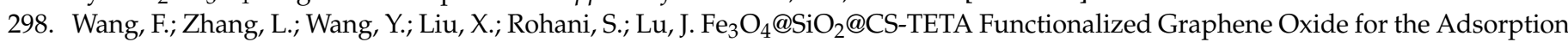
of Methylene Blue (MB) and Cu (II). Appl. Surf. Sci. 2017, 420, 970-981. [CrossRef]

299. Zhu, H.; Shen, Y.; Wang, Q.; Chen, K.; Wang, X.; Zhang, G.; Yang, J.; Guo, Y.; Bai, R. Highly Promoted Removal of Hg (II) with Magnetic $\mathrm{CoFe}_{2} \mathrm{O}_{4} @ \mathrm{SiO}_{2}$ Core-Shell Nanoparticles Modified by Thiol Groups. RSC Adv. 2017, 7, 39204-39215. [CrossRef]

300. Zou, Z.; Shi, Z.; Deng, L. Highly Efficient Removal of Cu (II) from Aqueous Solution Using a Novel Magnetic EDTA Functionalized $\mathrm{CoFe}_{2} \mathrm{O}_{4}$. RSC Adv. 2017, 7, 5195-5205. [CrossRef]

301. Lalhmunsiama; Pawar, R.R.; Hong, S.M.; Jin, K.J.; Lee, S.M. Iron-Oxide Modified Sericite Alginate Beads: A Sustainable Adsorbent for the Removal of As (V) and $\mathrm{Pb}$ (II) from Aqueous Solutions. J. Mol. Liq. 2017, 240, 497-503. [CrossRef]

302. Ren, C.; Ding, X.; Fu, H.; Li, W.; Wu, H.; Yang, H. Core-Shell Superparamagnetic Monodisperse Nanospheres Based on Amino-Functionalized $\mathrm{CoFe}_{2} \mathrm{O}_{4} @ \mathrm{SiO}_{2}$ for Removal of Heavy Metals from Aqueous Solutions. RSC Adv. 2017, 7, 6911-6921. [CrossRef]

303. Nithya, K.; Sathish, A.; Senthil Kumar, P.; Ramachandran, T. Fast Kinetics and High Adsorption Capacity of Green Extract Capped Superparamagnetic Iron Oxide Nanoparticles for the Adsorption of Ni (II) Ions. J. Ind. Eng. Chem. 2018, 59, 230-241. [CrossRef]

304. Silva-Yumi, J.; Escudey, M.; Gacitua, M.; Pizarro, C. Kinetics, Adsorption and Desorption of Cd (II) and Cu (II) on Natural Allophane: Effect of Iron Oxide Coating. Geoderma 2018, 319, 70-79. [CrossRef]

305. Guo, T.; Bulin, C.; Li, B.; Zhao, Z.; Yu, H.; Sun, H.; Ge, X.; Xing, R.; Zhang, B. Efficient Removal of Aqueous Pb (II) Using Partially Reduced Graphene Oxide-Fe ${ }_{3} \mathrm{O}_{4}$. Adsorpt. Sci. Technol. 2018, 36, 1031-1048. [CrossRef]

306. Mirzaeinejad, M.; Mansoori, Y.; Amiri, M. Amino Functionalized ATRP-Prepared Polyacrylamide-g-Magnetite Nanoparticles for the Effective Removal of Cu (II) Ions: Kinetics Investigations. Mater. Chem. Phys. 2018, 205, 195-205. [CrossRef]

307. Sun, J.; Chen, Y.; Yu, H.; Yan, L.; Du, B.; Pei, Z. Removal of $\mathrm{Cu}^{2+}, \mathrm{Cd}^{2+}$ and $\mathrm{Pb}^{2+}$ from Aqueous Solutions by Magnetic Alginate Microsphere Based on $\mathrm{Fe}_{3} \mathrm{O}_{4} / \mathrm{MgAl}$-Layered Double Hydroxide. J. Colloid Interface Sci. 2018, 532, 474-484. [CrossRef] 
308. Xu, W.; Song, Y.; Dai, K.; Sun, S.; Liu, G.; Yao, J. Novel Ternary Nanohybrids of Tetraethylenepentamine and Graphene Oxide Decorated with $\mathrm{MnFe}_{2} \mathrm{O}_{4}$ Magnetic Nanoparticles for the Adsorption of Pb (II). J. Hazard. Mater. 2018, 358, 337-345. [CrossRef] [PubMed]

309. Wang, Y.Y.; Ji, H.Y.; Lu, H.H.; Liu, Y.X.; Yang, R.Q.; He, L.L.; Yang, S.M. Simultaneous Removal of Sb (III) and Cd (II) in Water by Adsorption onto a $\mathrm{MnFe}_{2} \mathrm{O}_{4}$-Biochar Nanocomposite. RSC Adv. 2018, 8, 3264-3273. [CrossRef]

310. Wang, X.; Zhang, Z.; Zhao, Y.; Xia, K.; Guo, Y.; Qu, Z.; Bai, R. A Mild and Facile Synthesis of Amino Functionalized $\mathrm{CoFe}_{2} \mathrm{O}_{4} @ S i \mathrm{O}_{2}$ for $\mathrm{Hg}$ (II) Removal. Nanomaterials 2018, 8, 673. [CrossRef] [PubMed]

311. Song, T.; Yu, C.; He, X.; Lin, J.; Liu, Z.; Yang, X.; Zhang, Y.; Huang, Y.; Tang, C. Synthesis of Magnetically Separable Porous BN Microrods@ $\mathrm{Fe}_{3} \mathrm{O}_{4}$ Nanocomposites for Pb (II) Adsorption. Colloids Surfaces A Physicochem. Eng. Asp. 2018, 537, 508-515. [CrossRef]

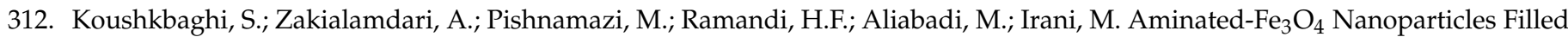
Chitosan/PVA/PES Dual Layers Nanofibrous Membrane for the Removal of $\mathrm{Cr}$ (VI) and Pb (II) Ions from Aqueous Solutions in Adsorption and Membrane Processes. Chem. Eng. J. 2018, 337, 169-182. [CrossRef]

313. Fu, W.; Huang, Z. One-Pot Synthesis of a Two-Dimensional Porous $\mathrm{Fe}_{3} \mathrm{O}_{4} /$ Poly $\left(\mathrm{C}_{3} \mathrm{~N}_{3} \mathrm{~S}_{3}\right)$ Network Nanocomposite for the Selective Removal of $\mathrm{Pb}$ (II) and $\mathrm{Hg}$ (II) from Synthetic Wastewater. ACS Sustain. Chem. Eng. 2018, 6, 14785-14794. [CrossRef]

314. Fu, W.; Huang, Z. Magnetic Dithiocarbamate Functionalized Reduced Graphene Oxide for the Removal of Cu (II), Cd (II), Pb (II), and $\mathrm{Hg}$ (II) Ions from Aqueous Solution: Synthesis, Adsorption, and Regeneration. Chemosphere 2018, 209, 449-456. [CrossRef]

315. Song, Y.; Lu, M.; Huang, B.; Wang, D.; Wang, G.; Zhou, L. Decoration of Defective MoS2 Nanosheets with Fe $\mathrm{O}_{4} \mathrm{Nanoparticles}$ as Superior Magnetic Adsorbent for Highly Selective and Efficient Mercury Ions $\left(\mathrm{Hg}^{2+}\right)$ Removal. J. Alloys Compd. 2018, 737, 113-121. [CrossRef]

316. Facchi, D.P.; Cazetta, A.L.; Canesin, E.A.; Almeida, V.C.; Bonafé, E.G.; Kipper, M.J.; Martins, A.F. New Magnetic Chitosan/Alginate $/ \mathrm{Fe}_{3} \mathrm{O}_{4} @ \mathrm{SiO}_{2}$ Hydrogel Composites Applied for Removal of $\mathrm{Pb}$ (II) Ions from Aqueous Systems. Chem. Eng. J. 2018, 337, 595-608. [CrossRef]

317. Huang, X.; Zhan, X.; Wen, C.; Xu, F.; Luo, L. Amino-Functionalized Magnetic Bacterial Cellulose/Activated Carbon Composite for $\mathrm{Pb}^{2+}$ and Methyl Orange Sorption from Aqueous Solution. J. Mater. Sci. Technol. 2018, 34, 855-863. [CrossRef]

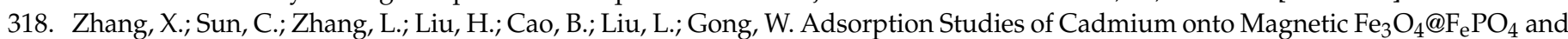
Its Preconcentration with Detection by Electrothermal Atomic Absorption Spectrometry. Talanta 2018, 181, 352-358. [CrossRef] [PubMed]

319. Banerjee, S.; Kumar, N.P.; Srinivas, A.; Roy, S. Core-Shell $\mathrm{Fe}_{3} \mathrm{O}_{4} @ \mathrm{Au}$ Nanocomposite as Dual-Functional Optical Probe and Potential Removal System for Arsenic (III) from Water. J. Hazard. Mater. 2019, 375, 216-223. [CrossRef]

320. Fu, W.; Wang, X.; Huang, Z. Remarkable Reusability of Magnetic $\mathrm{Fe}_{3} \mathrm{O}_{4}$-Encapsulated $\mathrm{C}_{3} \mathrm{~N}_{3} \mathrm{~S}_{3}$ Polymer/Reduced Graphene Oxide Composite: A Highly Effective Adsorbent for Pb and Hg Ions. Sci. Total Environ. 2019, 659, 895-904. [CrossRef]

321. Rowley, J.; Abu-Zahra, N.H. Synthesis and Characterization of Polyethersulfone Membranes Impregnated with (3Aminopropyltriethoxysilane) APTES- $\mathrm{Fe}_{3} \mathrm{O}_{4}$ Nanoparticles for As (V) Removal from Water. J. Environ. Chem. Eng. 2019, 7, 102875. [CrossRef]

322. Ramutshatsha-Makhwedzha, D.; Ngila, J.C.; Ndungu, P.G.; Nomngongo, P.N. Ultrasound Assisted Adsorptive Removal of Cr, $\mathrm{Cu}, \mathrm{Al}, \mathrm{Ba}, \mathrm{Zn}, \mathrm{Ni}, \mathrm{Mn}, \mathrm{Co}$ and Ti from Seawater Using $\mathrm{Fe}_{2} \mathrm{O}_{3}-\mathrm{SiO}_{2}-\mathrm{PAN}$ Nanocomposite: Equilibrium Kinetics. J. Mar. Sci. Eng. 2019, 7, 133. [CrossRef]

323. Zhang, L.; Guo, J.; Huang, X.; Wang, W.; Sun, P.; Li, Y.; Han, J. Functionalized Biochar-Supported Magnetic $\mathrm{MnFe}_{2} \mathrm{O}_{4} \mathrm{Nanocom}^{-}$ posite for the Removal of $\mathrm{Pb}$ (II) and Cd (II). RSC Adv. 2019, 9, 365-376. [CrossRef]

324. Xia, K.; Guo, Y.; Shao, Q.; Zan, Q.; Bai, R. Removal of Mercury (II) by EDTA-Functionalized Magnetic $\mathrm{CoFe}_{2} \mathrm{O}_{4} @ \mathrm{SiO}_{2} \mathrm{Nanomaterial}$ with Core-Shell Structure. Nanomaterials 2019, 9, 1532. [CrossRef]

325. Feng, G.; Ma, J.; Zhang, X.; Zhang, Q.; Xiao, Y.; Ma, Q.; Wang, S. Magnetic Natural Composite $\mathrm{Fe}_{3} \mathrm{O}_{4}$-Chitosan@bentonite for Removal of Heavy Metals from Acid Mine Drainage. J. Colloid Interface Sci. 2019, 538, 132-141. [CrossRef]

326. Wu, Z.; Deng, W.; Zhou, W.; Luo, J. Novel Magnetic Polysaccharide/Graphene Oxide @ $\mathrm{Fe}_{3} \mathrm{O}_{4}$ Gel Beads for Adsorbing Heavy Metal Ions. Carbohydr. Polym. 2019, 216, 119-128. [CrossRef]

327. Zendehdel, M.; Ramezani, M.; Shoshtari-yeganeh, B.; Salmani, A. Simultaneous Removal of Pb (II), Cd (II) and Bacteria from Aqueous Solution Using Amino-Functionalized $\mathrm{Fe}_{3} \mathrm{O}_{4}$ / NaP Zeolite Nanocomposite. Environ. Technol. 2019, 40, 3689-3704. [CrossRef] [PubMed]

328. Anush, S.M.; Vishalakshi, B. Modified Chitosan Gel Incorporated with Magnetic Nanoparticle for Removal of Cu (II) and Cr (VI) from Aqueous Solution. Int. J. Biol. Macromol. 2019, 133, 1051-1062. [CrossRef] [PubMed]

329. Xu, H.; Yuan, H.; Yu, J.; Lin, S. Study on the Competitive Adsorption and Correlational Mechanism for Heavy Metal Ions Using the Carboxylated Magnetic Iron Oxide Nanoparticles (MNPs-COOH) as Efficient Adsorbents. Appl. Surf. Sci. 2019, 473, 960-966. [CrossRef]

330. Fan, H.; Ma, X.; Zhou, S.; Huang, J.; Liu, Y.; Liu, Y. Highly Efficient Removal of Heavy Metal Ions by Carboxymethyl CelluloseImmobilized $\mathrm{Fe}_{3} \mathrm{O}_{4}$ Nanoparticles Prepared via High-Gravity Technology. Carbohydr. Polym. 2019, 213, 39-49. [CrossRef] [PubMed]

331. Zhang, H.; Tan, X.; Qiu, T.; Zhou, L.; Li, R.; Deng, Z. A Novel and Biocompatible $\mathrm{Fe}_{3} \mathrm{O}_{4}$ Loaded Chitosan Polyelectrolyte Nanoparticles for the Removal of $\mathrm{Cd}^{2+}$ Ion. Int. J. Biol. Macromol. 2019, 141, 1165-1174. [CrossRef] 
332. Al Yaqoob, K.; Bououdina, M.; Akhter, M.S.; Al Najar, B.; Vijaya, J.J. Selectivity and Efficient Pb and Cd Ions Removal by Magnetic $\mathrm{MFe}_{2} \mathrm{O}_{4}(\mathrm{M}=\mathrm{Co}, \mathrm{Ni}, \mathrm{Cu}$ and $\mathrm{Zn})$ Nanoparticles. Mater. Chem. Phys. 2019, 232, 254-264. [CrossRef]

333. Tahir, M.U.; Su, X.; Zhao, M.; Liao, Y.; Wu, R.; Chen, D. Preparation of Hydroxypropyl-Cyclodextrin-Graphene/Fe $\mathrm{O}_{4}$ and Its Adsorption Properties for Heavy Metals. Surf. Interfaces 2019, 16, 43-49. [CrossRef]

334. Zhou, G.; Wang, Y.; Zhou, R.; Wang, C.; Jin, Y.; Qiu, J.; Hua, C.; Cao, Y. Synthesis of Amino-Functionalized Bentonite/CoFe $\mathrm{O}_{4} @ \mathrm{MnO}_{2}$ Magnetic Recoverable Nanoparticles for Aqueous $\mathrm{Cd}^{2+}$ Removal. Sci. Total Environ. 2019, 682, 505-513. [CrossRef]

335. Masjedi, A.; Askarizadeh, E.; Baniyaghoob, S. Magnetic Nanoparticles Surface-Modified with Tridentate Ligands for Removal of Heavy Metal Ions from Water. Mater. Chem. Phys. 2020, 249, 122917. [CrossRef]

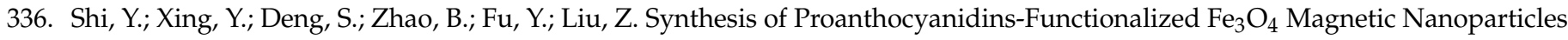
with High Solubility for Removal of Heavy-Metal Ions. Chem. Phys. Lett. 2020, 753, 137600. [CrossRef]

337. Kulal, P.; Badalamoole, V. Efficient Removal of Dyes and Heavy Metal Ions from Wastewater Using Gum Ghatti-Graft-Poly(4Acryloylmorpholine) Hydrogel Incorporated with Magnetite Nanoparticles. J. Environ. Chem. Eng. 2020, 8, 104207. [CrossRef]

338. Yoo, J.; Kim, H.S.; Park, S.Y.; Kwon, S.; Lee, J.; Koo, J.; Seo, Y.S. Instantaneous Integration of Magnetite Nanoparticles on Graphene Oxide Assisted by Ultrasound for Efficient Heavy Metal Ion Retrieval. Ultrason. Sonochem. 2020, 64, 104962. [CrossRef] [PubMed]

339. El-Dib, F.I.; Mohamed, D.E.; El-Shamy, O.A.A.; Mishrif, M.R. Study the Adsorption Properties of Magnetite Nanoparticles in the Presence of Different Synthesized Surfactants for Heavy Metal Ions Removal. Egypt. J. Pet. 2020, 29, 1-7. [CrossRef]

340. Zhang, X.; Huang, Y.; He, X.; Lin, J.; Yang, X.; Li, D.; Yu, M.; Yu, C.; Tang, C. Synergistic Adsorption of Pb (II) Ions by Fe ${ }_{3} \mathrm{O}_{4}$ Nanoparticles-Decorated Porous BN Nanofibers. Colloids Surf. A Physicochem. Eng. Asp. 2020, 589, 124400. [CrossRef]

341. Kamari, S.; Shahbazi, A. Biocompatible $\mathrm{Fe}_{3} \mathrm{O}_{4} @ \mathrm{SiO}_{2}-\mathrm{NH}_{2}$ Nanocomposite as a Green Nanofiller Embedded in PES-Nanofiltration Membrane Matrix for Salts, Heavy Metal Ion and Dye Removal: Long-Term Operation and Reusability Tests. Chemosphere 2020, 243, 125282. [CrossRef] [PubMed]

342. Pillai, P.; Kakadiya, N.; Timaniya, Z.; Dharaskar, S.; Sillanpaa, M. Removal of Arsenic Using Iron Oxide Amended with Rice Husk Nanoparticles from Aqueous Solution. Mater. Today Proc. 2020, 28, 830-835. [CrossRef]

343. Asadi, R.; Abdollahi, H.; Gharabaghi, M.; Boroumand, Z. Effective Removal of Zn (II) Ions from Aqueous Solution by the Magnetic $\mathrm{MnFe}_{2} \mathrm{O}_{4}$ and $\mathrm{CoFe}_{2} \mathrm{O}_{4}$ Spinel Ferrite Nanoparticles with Focuses on Synthesis, Characterization, Adsorption, and Desorption. Adv. Powder Technol. 2020, 31, 1480-1489. [CrossRef]

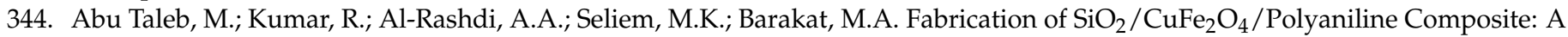
Highly Efficient Adsorbent for Heavy Metals Removal from Aquatic Environment. Arab. J. Chem. 2020, 13, 7533-7543. [CrossRef]

345. You, J.; Zhao, Y.; Wang, L.; Bao, W.; He, Y. Atomic Layer Deposition of $\gamma-\mathrm{Fe}_{2} \mathrm{O}_{3}$ Nanoparticles on Modified MWCNT for Efficient Adsorption of Cr (VI) Ions from Aqueous Solution. J. Phys. Chem. Solids 2020, 142, 109441. [CrossRef]

346. Zhou, Z.; Liu, X.; Zhang, M.; Jiao, J.; Zhang, H.; Du, J.; Zhang, B.; Ren, Z. Preparation of Highly Efficient Ion-Imprinted Polymers with $\mathrm{Fe}_{3} \mathrm{O}_{4}$ Nanoparticles as Carrier for Removal of $\mathrm{Cr}$ (VI) from Aqueous Solution. Sci. Total Environ. 2020, 699, 134334. [CrossRef]

347. Jafari, Z.; Avargani, V.M.; Rahimi, M.R.; Mosleh, S. Magnetic Nanoparticles-Embedded Nitrogen-Doped Carbon Nanotube/Porous Carbon Hybrid Derived from a Metal-Organic Framework as a Highly Efficient Adsorbent for Selective Removal of $\mathrm{Pb}$ (II) Ions from Aqueous Solution. J. Mol. Liq. 2020, 318, 113987. [CrossRef]

348. Xiao, J.; Hu, R.; Chen, G.; Xing, B. Facile Synthesis of Multifunctional Bone Biochar Composites Decorated with Fe/Mn Oxide Micro-Nanoparticles: Physicochemical Properties, Heavy Metals Sorption Behavior and Mechanism. J. Hazard. Mater. 2020, 399, 123067. [CrossRef] [PubMed]

349. Subana, P.S.; Manjunatha, C.; Manmadha, R.B.; Venkateswarlu, B.; Nagaraju, G.; Suresh, R. Surface Functionalized Magnetic $\alpha-\mathrm{Fe}_{2} \mathrm{O}_{3}$ Nanoparticles: Synthesis, Characterization and $\mathrm{Hg}^{2+}$ Ion Removal in Water. Surf. Interfaces 2020, 21, 100680. [CrossRef]

350. Devatha, C.P.; Shivani, S. Novel Application of Maghemite Nanoparticles Coated Bacteria for the Removal of Cadmium from Aqueous Solution. J. Environ. Manag. 2020, 258, 110038. [CrossRef] [PubMed]

351. Wang, H.; Wang, Z.; Yue, R.; Gao, F.; Ren, R.; Wei, J.; Wang, X.; Kong, Z. Functional Group-Rich Hyperbranched Magnetic Material for Simultaneous Efficient Removal of Heavy Metal Ions from Aqueous Solution. J. Hazard. Mater. 2020, $384,121288$. [CrossRef] [PubMed]

352. Mahanty, S.; Chatterjee, S.; Ghosh, S.; Tudu, P.; Gaine, T.; Bakshi, M.; Das, S.; Das, P.; Bhattacharyya, S.; Bandyopadhyay, S.; et al. Synergistic Approach towards the Sustainable Management of Heavy Metals in Wastewater Using Mycosynthesized Iron Oxide Nanoparticles: Biofabrication, Adsorptive Dynamics and Chemometric Modeling Study. J. Water Process Eng. 2020, $37,101426$. [CrossRef]

353. Kulal, P.; Badalamoole, V. Magnetite Nanoparticle Embedded Pectin-Graft-Poly(N-Hydroxyethylacrylamide) Hydrogel: Evaluation as Adsorbent for Dyes and Heavy Metal Ions from Wastewater. Int. J. Biol. Macromol. 2020, 156, 1408-1417. [CrossRef]

354. Shi, S.; Xu, C.; Wang, X.; Xie, Y.; Wang, Y.; Dong, Q.; Zhu, L.; Zhang, G.; Xu, D. Electrospinning Fabrication of Flexible Fe ${ }_{3} \mathrm{O}_{4}$ Fibers by Sol-Gel Method with High Saturation Magnetization for Heavy Metal Adsorption. Mater. Des. 2020, 186, 108298. [CrossRef]

355. Almomani, F.; Bhosale, R.; Khraisheh, M.; Kumar, A.; Almomani, T. Heavy Metal Ions Removal from Industrial Wastewater Using Magnetic Nanoparticles (MNP). Appl. Surf. Sci. 2020, 506, 144924. [CrossRef]

356. Hosain, A.N.A.; El Nemr, A.; El Sikaily, A.; Mahmoud, M.E.; Amira, M.F. Surface Modifications of Nanochitosan Coated Magnetic Nanoparticles and Their Applications in Pb (II), Cu (II) and Cd (II) Removal. J. Environ. Chem. Eng. 2020, 8, 104316. [CrossRef] 
357. Hou, L.; Liang, Q.; Wang, F. Mechanisms That Control the Adsorption-Desorption Behavior of Phosphate on Magnetite Nanoparticles: The Role of Particle Size and Surface Chemistry Characteristics. RSC Adv. 2020, 10, 2378-2388. [CrossRef]

358. Karvelas, E.G.; Lampropoulos, N.K.; Karakasidis, T.E.; Sarris, I.E. A computational tool for the estimation of the optimum gradient magnetic field for the magnetic driving of the spherical particles in the process of cleaning water. Desalination Water Treat. 2017, 99, 27-33. [CrossRef]

359. Peng, W.; Li, H.; Liu, Y.; Song, S. A Review on Heavy Metal Ions Adsorption from Water by Graphene Oxide and Its Composites. J. Mol. Liq. 2017, 496-504. [CrossRef]

360. Gautam, P.K.; Shivalkar, S.; Banerjee, S. Synthesis of M. Oleifera Leaf Extract Capped Magnetic Nanoparticles for Effective Lead [Pb(II)] Removal from Solution: Kinetics, Isotherm and Reusability Study. J. Mol. Liq. 2020, 305, 112811. [CrossRef]

361. Jadidi, M.; Etesami, N.; Nasr, E.M. Adsorption and Desorption Process of Chromium Ions Using Magnetic Iron Oxide Nanoparticles and Its Relevant Mechanism. Iran. J. Chem. Eng. 2017, 14, 31-40.

362. Lata, S.; Singh, P.K.; Samadder, S.R. Regeneration of Adsorbents and Recovery of Heavy Metals: A Review. Int. J. Environ. Sci. Technol. 2015, 12, 1461-1478. [CrossRef] 